\title{
A Parameterization Method for the Computation of Invariant Tori and Their Whiskers in Quasi-Periodic Maps: Explorations and Mechanisms for the Breakdown of Hyperbolicity*
}

\author{
A. Haro ${ }^{\dagger}$ and R. de la Llave
}

Abstract. In two previous papers [J. Differential Equations, 228 (2006), pp. 530-579; Discrete Contin. Dyn. Syst. Ser. B, 6 (2006), pp. 1261-1300] we have developed fast algorithms for the computations of invariant tori in quasi-periodic systems and developed theorems that assess their accuracy. In this paper, we study the results of implementing these algorithms and study their performance in actual implementations. More importantly, we note that, due to the speed of the algorithms and the theoretical developments about their reliability, we can compute with confidence invariant objects close to the breakdown of their hyperbolicity properties. This allows us to identify a mechanism of loss of hyperbolicity and measure some of its quantitative regularities. We find that some systems lose hyperbolicity because the stable and unstable bundles approach each other but the Lyapunov multipliers remain away from 1 . We find empirically that, close to the breakdown, the distances between the invariant bundles and the Lyapunov multipliers - which are natural measures of hyperbolicity-depend on the parameters, with power laws with universal exponents. We also observe that, even if the rigorous justifications in [J. Differential Equations, 228 (2006), pp. 530579] are developed only for hyperbolic tori, the algorithms work also for elliptic tori in Hamiltonian systems. We can continue these tori and also compute some bifurcations at resonance which may lead to the existence of hyperbolic tori with nonorientable bundles. We compute manifolds tangent to nonorientable bundles.

Key words. invariant tori, invariant manifolds, quasi-periodic systems

AMS subject classifications. 37M99, 37D10, 70K40, 70K43

DOI. $10.1137 / 050637327$

1. Introduction. In this paper we implement the numerical algorithms for the computation of invariant tori and their whiskers in quasi-periodically forced systems presented in [HdlL05a]. The whiskers include in particular the stable and unstable manifolds, but also the nonresonant and slow invariant manifolds attached to the whiskers.

The algorithms are based on the parameterization method introduced in [HdlL06b] for quasi-periodic systems (see also [CFdlL03a, CFdlL03b, CFdlL05] for the parameterization

\footnotetext{
${ }^{*}$ Received by the editors August 1, 2005; accepted for publication (in revised form) by T. Kaper September 11, 2006; published electronically March 15, 2007.

http://www.siam.org/journals/siads/6-1/63732.html

${ }^{\dagger}$ Departament de Matemàtica Aplicada i Anàlisi, Facultat de Matemàtiques, Universitat de Barcelona, Gran Via de les Corts Catalanes 585, 08007 Barcelona, Spain (alex@maia.ub.es). This work was initiated when this author was enjoying a Fulbright Scholarship at The University of Texas at Austin in the year 2000. He has been supported by the MCyt/FEDER grant BFM2000-805 and the Catalan grant 2000SGR-27 and the INTAS project 00-221. His work is also supported by the MCyT/FEDER grant BFM2003-07521-C02-01.

${ }^{\ddagger}$ Dept. of Mathematics, The University of Texas at Austin, 1 University Station C1200, Austin, TX 78712-0257 (llave@math.utexas.edu). The work of this author has been partially supported by N. S. F. grants. He enjoyed a Dean's Fellowship at U.T. Austin in spring of 2003. Visits of this author to Barcelona were supported by FBBV and ICREA.
} 
method in other contexts).

Using the numerical procedures developed, we analyze several examples that have been considered in the literature in regimes where the results of [HdlL06b] apply.

Since the results in [HdlL06b] give "a posteriori estimates," we know that, in some regimes, the numerical computations correspond to true solutions of the problem close by. We will also use the term "validating" as a synonym for "a posteriori."

It is important to realize that once we have validating results, to guarantee the validity of the calculations, we do not need to study the algorithms. We just need to check-after the calculation is done - that the calculation leads to objects that solve the desired equation with a small error and that they satisfy the nondegeneracy assumptions required for the theorem. Of course, an analysis of the algorithms may be useful for obtaining an idea of how many steps or what number of modes will be needed.

Once the algorithms have been benchmarked in regions where they are backed up by a rigorous analysis, we explore situations which are not yet covered by a rigorous analysis. This allows us to formulate conjectures, which we hope will stimulate new analytical results. The main empirical findings of this exploration are as follows.

- We have identified an apparently robust scenario for the breakdown of exponential dichotomies and, in particular for the breakdown of hyperbolicity.

In this "bundle merging" scenario, the invariant bundles approach each other and their distance tends to zero as the parameters approach a critical value. Nevertheless, before the critical value, the spectrum remains uniformly separated.

We have identified several scaling properties in this scenario and we have found that, in the examples considered, the exponents of these scaling laws are universal.

In sections 3.2 and 6 we report in detail these phenomena. See also [HdlL06a].

- Even if the mathematical justifications in [HdlL06b] are stated only for normally hyperbolic tori, we note that some of the algorithms in [HdlL05a] work for tori whose linearizations are elliptic in the normal directions.

Hence, we can continue these tori with respect to parameters of the map. This has already been done in [CJ00], using the Newton method. In this paper we also use algorithms based on reducibility. The refined numerical study here allows us to explore bifurcations that happen when there are resonances between the normal modes and the internal modes. This leads to a bifurcation in which the torus becomes hyperbolic. We note that, in this case, the stable and unstable bundles of the tori may be nonorientable. The appearance of nonorientable bundles in applications is the main reason why the algorithms developed in the first part took special pains to include nonorientable bundles.

There are justifications of persistence of elliptic tori in the literature [Eli88, JS96, JV97, Pös89], in rather general contexts, but they are not designed to validate numerics. An a posteriori result that can be found in a slightly more restrictive context is in [JdlLZ99]. We hope that an a posteriori result will be available in the near future. Some results on the bifurcations of elliptic tori at resonance are available in $\left[\mathrm{BHJ}^{+} 03\right]$, but they do not include the study of the hyperbolic manifolds generated. We hope that an a posteriori result tailored toward our numerics will be available soon.

1.1. Quasi-periodic maps. The examples we study in this paper are quasi-periodic perturbations of two-dimensional (2D) maps. We will take the external perturbation to be quasi- 
periodic with one frequency. That is, we will consider maps of the form

$$
\left(\begin{array}{l}
x \\
y \\
\theta
\end{array}\right) \longrightarrow\left(\begin{array}{c}
f_{\varepsilon}(x, y, \theta) \\
g_{\varepsilon}(x, y, \theta) \\
\theta+\omega
\end{array}\right)
$$

where $x, y \in \mathbb{R}$ or $\mathbb{T}$ are the state variables, $\theta \in \mathbb{T}$ is the external angle, and $\varepsilon$ is a parameter (for $\varepsilon=0$, the functions $f_{0}, g_{0}$ do not depend on $\theta$ ). The frequency $\omega \in \mathbb{R}$ is supposed to be irrational (in fact, we assume that $\omega$ is Diophantine, although for some of the algorithms and results this extra assumption is not necessary). By introducing the $2 \mathrm{D}$ variable $z=(x, y)$, we write (1) in a more compact way as

$$
\left(\begin{array}{c}
z \\
\theta
\end{array}\right) \longrightarrow\left(\begin{array}{c}
F_{\varepsilon}(z, \theta) \\
\theta+\omega
\end{array}\right) \text {. }
$$

This is the simplest nontrivial case, and it is a natural first step to test the methods. It seems that adding more variables to the phase space will not be too complicated, but we thought it would be better to study in detail some new phenomena that appear already. Of course, we plan to come back to these issues.

Maps of the form (2) appear also as the surface of section maps of flows which are quasiperiodically perturbed. In this case, $\omega$ is the ratio of the two external frequencies. We will not present any specific examples of flows (see [CJ00]). The papers [HdlL06b, HdlL05a] present some algorithms that are specifically designed for flows.

The examples we consider are quasi-periodic perturbations of two very well-known maps: the Hénon map and the standard map. We have included examples in which the perturbations are analytic and another one in which the perturbation is just Lipschitz. As we will see in the examples, the algorithms yield high precision results (in some cases, the errors are a few times the order of magnitude of the machine epsilon). We will see that the algorithms can handle $10^{5}$ Fourier coefficients in hours of work of a by now outdated desktop computer. (High order expansions are required in the study of the Lipschitz example here, but a large number of Fourier coefficients are needed in the computation of higher dimensional invariant tori.)

1.2. Some similar calculations in the literature. Let us end this introductory section recalling some of the areas in which the computation of invariant manifolds has proved fruitful. Since invariant manifolds organize the global behavior of a dynamical system, their importance is both theoretical and practical. We will focus on the practice in this paper. For instance, codimension 1 invariant manifolds are barriers in the phase space and are useful in describing transport and formation of resonances. For 2D systems, stable and unstable manifolds of hyperbolic periodic orbits have been used to study the geometrical structure of the attractors in the Hénon map [Sim79], the mechanisms of destruction of invariant tori for the standard map [OS87], transport phenomena in area preserving maps [Mei92], etc. In four dimensions, the stable and unstable manifolds of a center manifold of a hyperbolic-elliptic fixed point of the Froeschlé map have been used to bound a stability region around the totally elliptic fixed point [Har99].

In celestial mechanics, stable and unstable manifolds of quasi-periodic solutions in the Solar System can be used also to perform transfer orbits in space missions [Sim99, GJMS91b, GJMS91a, ESA, NAS]. The list of applications is far from complete. 
We mention some papers dealing with a quasi-periodically forced system. In [BLW91], the whiskers of invariant tori are the tool used to explain transport in the homoclinic and heteroclinic tangle regions in the quasi-periodic $2 \mathrm{D}$ case. In [OF00] these manifolds explain the structure of the attractors in a quasi-periodically forced Hénon map (what we call the rotating Hénon map), and the computational method is based on a general algorithm [BOV97]. In these papers, the manifolds are approximated using simplicial complexes. The papers [LM00, LM03] compute invariant manifolds for volume preserving maps. In [Har05], these manifolds are related to the breakdown of KAM tori in a quasi-periodically forced standard map (what we call the rotating standard map).

Since the objects we compute are invariant tori (and their whiskers), we will use coordinates that are more adapted to them. It is natural to parameterize invariant tori using angular coordinates, so the functions are periodic in the angular coordinates and we use truncated Fourier series in their approximations. It is also natural to parameterize invariant manifolds attached to invariant tori using angular Cartesian coordinates, and then use truncated Fourier-Taylor series in their approximations.

The use of truncated Fourier series to compute invariant tori using Newton's method for the invariance equation appears, for instance, in [WR87, Har02] (KAM tori), [dlLT94, CJ00] (invariant tori in quasi-periodic systems), [GJ04] (lower dimensional tori in autonomous conservative systems), and [MKM97] (PDE approach in continuous systems). Other different approaches to compute invariant tori can be found, for instance, in [DLR91, DL95, Sim98, ERS00, Tru00].

We also note that the use of high order expansions (Taylor, Fourier, Fourier-Taylor, Lindstedt, etc.) to compute invariant manifolds has been standard in the work of C. Simó since the 1970's and it has been used in many of his papers, often without mention.

2. Some implementation details. In this section, we provide some details about the implementation we have carried out.

At the moment, we have a rather complete toolkit to manipulate periodic functions of one angle variable. This allows us to consider, rather comfortably, one-dimensional (1D) invariant tori and their whiskers. The numerical results reported in this paper are only for the case that the ambient space is three-dimensional (3D) (2D maps are subject to a quasi-periodic perturbation of one frequency).

The restriction to two dimensions allows some mathematical simplifications, and allows more complete visualization. It is a natural first step. Increasing the dimension of the maps seems rather straightforward but increasing the dimension of the tori requires more effort. Both of these improvements are actively pursued.

2.1. The parameterization equations for tori and their whiskers. In this section, we recall concisely the main algorithms developed in [HdlL05a].

2.1.1. Equations for invariant tori and algorithms to solve them. Following the parameterization method, to find a $1 \mathrm{D}$ torus invariant for (1) we seek a mapping $K: \mathbb{T} \rightarrow \mathbb{R}^{2}$ satisfying

$$
F(K(\theta), \theta)=K(\theta+\omega) .
$$


In [HdlL05a] we consider several algorithms to solve (3). For the purposes of this paper, the only one we will consider is the following:

- Newton method. Given a $K$ that approximately solves (3), we obtain $\hat{K}$, an improved approximate solution of (3), by solving the linearization of (3).

More explicitly, if $K$ satisfies

$$
r(\theta) \equiv F(K(\theta-\omega), \theta-\omega)-K(\theta)
$$

(we should think of $r$ as the error), an improved solution is $\tilde{K}=K+h$, where $h$ is obtained by solving

$$
\mathrm{D} F(K(\theta-\omega), \theta-\omega) h(\theta-\omega)-h(\theta)=-r(\theta) .
$$

The equations (4), which define the Newton method, can be treated in different ways.

- Large matrix method. The most straightforward method is simply to discretize (4) in a basis of discretization and then apply a linear equation solver.

This method is relatively easy to implement and is quite robust. Even if the justifications of this method in [HdlL05a] are for normally hyperbolic tori, we have found empirically that the Newton method also works well for normally elliptic tori in Hamiltonian systems. See section 4.3 and [CJ00].

Even if this procedure is adequate for many problems, it has the disadvantage that it scales badly with the number of discretization parameters. If we discretize the equation using $N$ coefficients, the storage required for a full matrix is $N^{2}$ and the number of operations in the inversion of a matrix to accomplish one step of Newton method $O\left(N^{3}\right)$. We refer to this shortcoming as the large matrix problem.

In slightly out of date desktop computers, the method works very well for $N$ being a few hundreds, even about one thousand, but it quickly becomes impractical.

The following algorithms overcome this large matrix problem, are faster, and use less storage. In section 5 we present an example of a problem which is very impractical for the large matrix method.

- Projection method. This method works for normally hyperbolic tori. The first step is to seek parameterizations of the stable and unstable bundles. In the case that they are $1 \mathrm{D}$, this can be accomplished just by the linearized equation.

Notice that to store the $\mathrm{D} F \circ K(\theta)$ by storing the matrix elements, we need only $O(N)$ storage. One step of application of $\mathrm{DF}$ requires just the multiplication of several functions. As we will see later, much of the work reported here is done with algorithms of multiplications which require $O\left(N^{2}\right)$ operations (after considering several issues we decided not to use multiplication algorithms in [Knu97] which are theoretically $O\left(N^{1+\delta}\right)$ or even $O(N \log N)$ ). In any case, irrespective of which multiplication algorithm is used, the application of DF is significantly faster than the inversion of a matrix.

Once we have the stable and unstable bundles, we can take the projection of the error into them and solve the resulting equations by iterations. The rate of convergence of the method depends on the size of the gap between the stable and unstable modes.

- Reducibility method. This method tries not only to solve (3) but also to find a constant 
matrix $\Lambda$ and a periodic matrix $P(\theta)$ such that

$$
P(\theta+\omega)^{-1} M(\theta) P(\theta)-\Lambda=0,
$$

where $M(\theta)=\mathrm{D} F(K(\theta), \theta)$. In this case, we say that the torus is reducible.

Note that having a solution of (5) makes it very easy to solve (4). If we substitute (5) into (4), it becomes

$$
\Lambda P^{-1}(\theta) h(\theta)-P^{-1}(\theta+\omega) h(\theta+\omega)=-P^{-1}(\theta+\omega) r(\theta) .
$$

Equation (6) is an equation for $H(\theta)=P^{-1}(\theta) h(\theta)$, which, as we will see, is blockdiagonal in a Fourier series; hence it can be solved very quickly if we discretize in Fourier series. The desired function $h$ can be obtained by multiplying $h(\theta)=$ $P(\theta) H(\theta)$. The storage of $P, P^{-1}, \mathrm{D} F$, etc. is only $O(N)$ and operations required are only linear in $N$ and multiplications of Fourier series so that they are much faster than inverting full matrices.

For the sake of simplicity, assume that $\Lambda=\operatorname{diag}\left(\lambda_{1}, \lambda_{2}\right)$ is diagonal. Then, denoting $R(\theta)=-P^{-1}(\theta+\omega) r(\theta)$ and denoting the Fourier coefficients by subindices separated by ; from the indices denoting the vector components, (6) becomes in Fourier terms

$$
\left(\lambda_{j}-\exp (2 \pi \mathbf{i} k \omega)\right) H_{j ; k}=R_{j ; k} .
$$

In summary, if we know $P$ solving (5), we can reduce the solution of (4) to several multiplication of Fourier series and to a solution of (7), which is linear in the number of terms used in the discretization.

Equation (5) can be treated also via a Newton method, which as we will see can be given a fast implementation in terms of Fourier discretizations. We give some details. Given a $P, \Lambda$ for which

$$
P(\theta+\omega)^{-1} M(\theta) P(\theta)-\Lambda=S(\theta),
$$

with $S$ small, we seek improved solutions of the form $\hat{P}=P(\operatorname{Id}+Q), \hat{\Lambda}=\Lambda+\Delta$, with $\Delta=\operatorname{diag}\left(\delta_{1}, \delta_{2}\right)$.

Expanding (5) up to first order in the corrections we obtain

$$
\Lambda Q(\theta)-Q(\theta+\omega) \Lambda-\Delta=-S(\theta) .
$$

Equation (8) is diagonal in Fourier coefficients so that it can be solved in a time proportional to the number of coefficients used in the discretization. Equation (8) in Fourier coefficients amounts to

$$
\begin{aligned}
& \left(\lambda_{i}-\exp (2 \pi \mathbf{i} k \omega) \lambda_{j}\right) Q_{i, j ; k}=-S_{i, j ; k} \quad \text { when } k \neq 0 \text { or } i \neq j ; \\
& Q_{i, i ; 0}=0, \delta_{i}=S_{i, i ; 0} .
\end{aligned}
$$

In practice, since inverting a Fourier matrix from scratch is time consuming, we keep an extra matrix $\bar{P}(\theta)$ which is supposed to be the inverse of the matrix $P(\theta)$, that is,

$$
\bar{P}(\theta) P(\theta)-\mathrm{Id}=0 .
$$


When $P$ changes, it is very easy to obtain the new $\bar{P}$ as a perturbation of the other one. Hence, we write a Newton method for $K, P, \bar{P}, \Lambda$ [HdlL05a].

In [HdlL06b] it is shown that if one uses a Newton step for both (3) and (5), one obtains a method which solves both equations with quadratic convergence. This requires $O(N)$ storage and the cost of one step of the method is comparable to the cost of one multiplication.

Remark 2.1. The theory has to consider several normalizations that take care of the fact that the solution of (5) is not unique. For example, multiplying $P$ by a constantor by any matrix that commutes with $\Lambda$-is also a solution.

Nevertheless, the algorithm described above converges to a solution of (5), which is all that is needed.

We note that the reducibility method also gives us very detailed information on the invariant subspaces.

We also note that we have found empirically that this method works well when $\lambda_{1}, \lambda_{2}$ are complex imaginary numbers of modulus 1 for which the small divisors in (9) and in (7) do not vanish except for $k$ moderately large. This situation happens for elliptic tori in Hamiltonian systems. The empirical results are presented in section 4.3.

On the other hand, we note that it can happen that (5) may fail to have solutions even if the system is hyperbolic (and, therefore, the projection method works), so that the reducibility method, even if more effective, has a more reduced range of applicability. The phenomena that happen when (5) fails are quite interesting. We will study these failures in sections 3.2 and 6.

Our most efficient method to continue tori along a parameter is to use the reducibility method whenever possible but keep an eye on when (5) runs into trouble. Near these places, the method switches to the projection method or the large matrix method. Since the later methods require more effort, they may have to be run with fewer Fourier coefficients.

Remark 2.2. The condition that the coefficients of the unknowns that appear in (7) do not vanish is usually called the first Melnikov condition. The condition that the coefficients of the unknowns that appear in (9) do not vanish is usually called the second Melnikov condition.

Remark 2.3. We will see that, in this $2 \mathrm{D}$ context, reducibility is closely related to spectral properties of the transfer operator. In particular, it is closely related to the fact that the spectrum of the transfer operator consists of two circles. See section 2.2.5.

We will see that, in the dissipative context, there are quasi-periodic 2D mappings for which reducibility fails because of topological reasons. Similar examples happen in four-dimensional (4D) symplectic mappings. There are also intervals in which reducibility seems to be very hard to compute numerically. In these intervals, there seem to be hierarchies of subintervals in which the reducibility is harder and harder to compute. Moreover several topological and geometric properties seem to change in each level of the hierarchy. See section 6.3.4.

On the other hand, one can expect that reducibility is quite abundant among elliptic situations. In [dlLGJV05] it is shown that Lagrangian KAM tori are reducible, so that the algorithms derived here work also in this case with slight modifications.

2.1.2. Equations for invariant whiskers. In this paper we will be interested only in the study of whiskers which have one dimension more than the tori. We will also restrict ourselves 
to the nonresonant case discussed in [HdlL06b]. This includes as particular cases the stable and unstable manifolds in a saddle-type torus and the fast stable manifolds. We also include the slow stable manifolds in case there is no resonance.

We will look for a parameterization for the torus given by an angle variable that measures the position with respect to the torus and another variable that moves in a direction roughly along the bundle. We will require that, in the chosen parameterization, we have that the angle variable moves by a rigid rotation and the normal variable contracts linearly. The fact that these normalizations can be done without loss of generality is discussed in [HdlL06b].

Hence, we will be looking for functions $W: \mathbb{T}^{1} \times \mathbb{R}^{1} \rightarrow \mathbb{R}^{2}$ in such a way that

$$
F(W(s, \theta), \theta)=W(\lambda s, \theta+\omega),
$$

where the unknowns are both $W$ and $\lambda$, with $|\lambda| \neq 1$.

This equation can be adapted also to deal with nonorientable whiskers. As we will see, this generality is natural since in section 4.3 we will see that nonorientable bundles appear naturally in the bifurcations of elliptic tori. To deal with nonorientable bundles we just consider

$$
F\left(W\left(s, \frac{1}{2} \theta\right), \theta\right)=W\left(\lambda s, \frac{1}{2}(\theta+\omega)\right) .
$$

Equation (11) will be discretized in Fourier-Taylor series. We will write

$$
W(s, \theta)=\sum_{i=0} W_{i}(\theta) s^{i}
$$

and match similar coefficients in $s^{i}$ on both sides of (11).

We obtain

$$
\begin{aligned}
& F\left(W_{0}(\theta), \theta\right)=W_{0}(\theta+\omega), \\
& \operatorname{DF}\left(W_{0}(\theta), \theta\right) W_{1}(\theta)=\lambda W_{1}(\theta+\omega) \\
& \cdots \\
& \operatorname{DF}\left(W_{0}(\theta), \theta\right) W_{k}(\theta)+S_{k}\left[W_{0}, W_{1}, \ldots, W_{k-1}\right](\theta)=\lambda^{k} W_{1}(\theta+\omega),
\end{aligned}
$$

where $S_{k}$ is an explicit polynomial in $W_{0}, W_{1}, \ldots, W_{k-1}$ whose coefficients are derivatives of $F$ evaluated at $W_{0}$. The polynomials $S_{k}$ can be readily computed using the methods of automatic differentiation or series manipulation.

The first equation admits the solution $W_{0}=K$, where $K$ is the parameterization of an invariant circle. (That is, we are choosing an invariant circle to study.)

The second equation tells us that $\lambda$ is an eigenvalue of the transfer operator defined by

$$
\mathcal{M}_{\omega} V(\theta)=M(\theta-\omega) V(\theta-\omega)
$$

and that $W_{1}$ is an eigenvector. This equation determines the space that we are choosing. This determines $\lambda$ and determines $W_{1}$ up to a multiple. Even if all the choices of $W_{1}$ are mathematically equivalent (see [HdlL06b, HdlL05a]) and correspond to the nonuniqueness of 
the solutions of (11), the choice affects the numerical properties of the algorithm. This will be discussed in more detail in section 2.1.5.

The other equations are quite straightforward. Note that, proceeding by induction, we can assume that $W_{0}, \ldots, W_{k-1}$ are known. The resulting equations for $W_{k}$ can be solved provided that $\lambda^{k}$ is not an eigenvalue of the transfer operator, which is the main assumption in the theorems about existence of nonresonant whiskers in [HdlL06b]. See also [CFdlL03a, dlL97].

We note that the nonresonance assumption above is automatically satisfied for the strong stable manifolds (resp., the strong unstable manifolds) of an attractive (resp., repelling) torus or by the stable and unstable manifolds of a saddle-type torus. In [HdlL06b] it is shown that all the classical manifolds just mentioned can be obtained through a parameterization as above. The only thing to check is that given a classical manifold, there are some coordinates in which the motion can be made to be a multiplication by a number. In [HdlL06b] we also consider cases in which reduction to a multiplication is not possible, but reduction to a polynomial dynamics is possible.

2.1.3. Fourier-Taylor series. Since the components of the parameterization of a $1 \mathrm{D}$ torus are periodic functions of one angle variable $\theta$, it is natural to consider Fourier series [dlLT94, CJ00]. We store the periodic functions of one variable as cosine and sine Fourier series. If $f(\theta)$ is a 1-periodic function, we will write

$$
f(\theta)=a_{0}+\sum_{k>0}\left(a_{k} \cos (2 \pi k \theta)+b_{k} \sin (2 \pi k \theta)\right) .
$$

The finite computer memory forces us to cut off these expansions. The objects that we compute are smooth, or even analytic; hence, the coefficients in these expansions decrease eventually faster than powers or even faster than exponentially. Of course, when we move parameters so that the torus is close to breakdown, this eventually fast convergence will only be apparent for the coefficients of very high order. In this paper we also present an example of a Lipschitz map whose invariant torus is only Lipschitz, so the Fourier coefficients of its parameterization decrease very slowly.

The norm we have used in order to estimate the errors in the computations is

$$
\|f\|=\left|a_{0}\right|+\sum_{k>0} \sqrt{a_{k}^{2}+b_{k}^{2}} \geq\|f\|_{\infty} .
$$

The numbers $c_{k}=\sqrt{a_{k}^{2}+b_{k}^{2}}$ are called the amplitudes of the Fourier modes. For a periodic matrix $M(\theta)=\left(M_{i, j}(\theta)\right)$, we denote $\|M\|=\max _{i, j}\left\|M_{i, j}\right\|$.

For the computation of whiskers of invariant tori, we use Fourier-Taylor expansions of the form

$$
f(s, \theta)=\sum_{m=0}^{\infty} f_{m}(\theta) s^{m},
$$

with

$$
f_{m}(\theta)=a_{m, 0}+\sum_{k>0}\left(a_{m, k} \cos (2 \pi k \theta)+b_{m, k} \sin (2 \pi k \theta)\right)
$$


We have used a package of routines that perform operations on series as above. Certainly, it is elementary to implement the elementary algebraic functions (sum, product, and product by scalar), since there are ready formulas for the coefficients of the result in terms of the coefficients of each of the terms. It is not too hard to implement the division of series and elementary transcendental functions (exp, sin, cos, log, power, etc.). Some hints on how to implement the elementary transcendental functions are given in [Knu97]. These techniques are known under the name of automatic differentiation algorithms. Similar packages have been implemented many times in the literature [GC91, RJB83, MS91, Koc99, Har02].

Remark 2.4. The most straightforward algorithm for multiplication of Fourier series is the use of the Cauchy formula. If we implement it in the most straightforward way, evaluating the product of two series of $N$ coefficients requires $O\left(N^{2}\right)$ operations to complete.

In [Knu97], one can find a discussion of algorithms that can be asymptotically faster. The asymptotically fastest is to compute the FFT, multiply, and then take the FFT back. This would give an asymptotic cost of $O(N \log (N))$. Preliminary testing suggests that these different methods have different numerical properties.

In this package we have used always the straightforward Cauchy formula.

It would be quite natural to implement the Fourier manipulation routines using the BLAS library. We have not done so, but rather have used explicit loops. This has the advantage that the precision of the programs can be changed from float to double to long double very easily.

Using these routines, one can implement the operators involved in (3), (11), and their derivatives provided that the function $F$ can be written using algebraic operations and the elementary transcendental functions.

If the map is not given explicitly (for instance, if it is given by the flow of a vector field) or its computation is hard, we could follow the following strategy [CJ00]: take a mesh of points on the torus, apply the map to the mesh of points, and compute the torus corresponding to the image of the new mesh by applying FFT methods. To compute first derivatives (the first order terms in the Taylor expansions), one has to integrate the first order variational equations of the flow [CJ00]. For higher order derivatives, one has to integrate higher order variational equations.

For flows, one can also use functional equations to determine the invariant manifolds. The invariance equation of a torus is a first order linear PDE [DLR91, DL95, MKM97, ERS00, Tru00]. See also [HdlL06b] for the invariance equations for invariant tori and their whiskers in quasi-periodic differential equations.

As the examples that we have studied here are maps that involve only a few operations in this paper we have used only the Fourier-Taylor methods. We think that a detailed comparison between the FFT method and the Fourier-Taylor method would be quite interesting, and we plan to come back to this issue. Preliminary studies indicate that the numerical properties of both methods are quite different, especially if one looks at properties which involve derivatives.

We have not undertaken a comparison of the Fourier-Taylor method with other methods of discretization (e.g., splines). On the other hand, we note the following.

- As will be seen in the section on numerical results, we will routinely obtain residuals which are of the order $10^{-15}-10^{-20}$ using only 100 terms or so (and the error is possibly an overestimate). Achieving a similar accuracy would seem to require a method of 
interpolation of rather high degree whose stability properties would be problematic.

- Many of the equations we have to solve in a Newton step are diagonal - or quasidiagonal - when discretized in Fourier series. In particular, the reducibility method, when discretized in Fourier series, makes the computational effort of a Newton step essentially linear in the number of terms.

- Fourier methods have the disadvantage that they are not adaptive and it is hard to increase the accuracy in the places where the manifold becomes more oscillatory.

In the present examples, the fact that the dynamics on the torus is a rigid rotation makes the torus very homogeneous, so that there is little need for adaptivity. Our implementation can increase or decrease the number of Fourier terms used dynamically.

The above reasons, especially the last two, are very closely tied to the models that we are considering in this paper. It is quite possible that, when applying the parameterization method to other problems, other methods could prove to be more convenient.

2.1.4. Assessment of the error of the calculation of invariant tori. We have introduced several measurements that give an idea of what accuracy is achieved by our calculations of invariant tori and their invariant bundles.

Given $K, P, \bar{P}, \Lambda$ approximate solutions of the equations of the invariance equation (3), the reducibility equation (5), and the inverse equation (10), we give names to the remainders as follows:

$$
\begin{aligned}
R(\theta) & =\bar{P}(\theta+\omega)(F(K(\theta), \theta)-K(\theta+\omega)), \\
S(\theta) & =\bar{P}(\theta+\omega) \operatorname{DF}(K(\theta), \theta) P(\theta)-\Lambda(\theta), \\
T(\theta) & =\bar{P}(\theta) P(\theta)-\mathrm{Id} .
\end{aligned}
$$

The quality of the approximate solutions is given by the norms of these Fourier matrices, as defined in section 2.1.3.

Remark 2.5. If we apply the Newton method, then we take $P=\bar{P}=\operatorname{Id}$ and $\Lambda(\theta)=$ $\mathrm{DF}(K(\theta), \theta)$, so $S=0$ and $T=0$.

2.1.5. Domains of the parameterization of the whiskers. We have explained in [HdlL06b, HdlL05a] how to compute the Fourier-Taylor expansions of the parameterizations of the whiskers. Given such an expansion, we have to obtain a domain in which such approximations are good enough to allow reliable evaluations.

In principle, the parameterization gives a complete description of the manifold and the parameterization can follow the twists and turns of the manifold.

In practice, however, the numerical evaluation could become numerically unstable at larger values of $s$. Hence, it is sometimes more advantageous to evaluate $W$ only for values of $s$ smaller than a certain $s_{0}$ and then use the functional equation (11).

We refer to this last step as globalizing the manifold. This has been always an ingredient of algorithms to compute invariant manifolds since all the algorithms give only some local piece. See, for example, [KO98]. We note that, in our case, since the parameterization covers, in principle, the whole manifold, the globalization is to a large extent a matter of numerical convenience and that even without performing any extension, the parameterization can follow a large number of turns in the manifold. 
Remark 2.6. We note that if $W(s, \theta)$ is a solution of $(11)$, so is $\tilde{W}(s, \theta)=W(r \cdot s, \theta)$ for any $r \in \mathbb{R}$. The choice of $r$ is closely related to the choice of the first coefficient in the expansion. Hence, the choice of the multiple in the first step is related to the domain. From the mathematical point of view, it is equivalent to choosing a larger $W_{1}$ and then evaluating in a proportionally small domain. Nevertheless, from the numerical point of view, both methods are inequivalent. If we have coefficients of different sizes, they are much more prone to roundoff error. Hence, a rule of thumb is to choose $W_{1}$ so that the coefficients computed have comparable sizes. In practice, one can do a preliminary run that gives some idea of how the coefficients grow. Since choosing $\rho W_{1}$ instead of $W_{1}$ makes $W_{k}$ become $\rho^{k} W_{k}$, it is easy to choose $\rho$ so that the coefficients $W_{k}$ have a comparable size. Similar adjustments are common also in Lindstedt series [FdlL92].

Remark 2.7. The computation of slow manifolds [CFdlL03a, CFdlL03b, CFdlL05] deserves a few words. Notice that the dynamics around an attracting invariant circle is dominated by the normal directions associated to the "eigenvalue" with largest modulus. The situation is analogous to the situation in linear maps. The components along the most contractive eigenvalues disappear faster than those along the slow eigenvalue and the component along the slow eigenvalue is the dominant one for long term behavior. Hence, from the point of view of asymptotic behavior, the manifold associated to the spectral values closest to the unit circle is the most observable. In [HdlL06b] it is shown that under some appropriate nonresonance conditions, the nonresonant manifold exists and is unique among the tangent manifolds of a degree of regularity that depends only on the spectrum. Then, this unique manifold is as smooth as the map. Hence, as soon as we know that there is one invariant manifold with a moderate regularity, we can bootstrap the regularity to the regularity of the map. These results are sharp because examples in [HdlL06b] show that there are examples with resonances with no smooth invariant manifolds. Moreover, there may be many invariant manifolds with low regularity.

In the $2 \mathrm{D}$ set-up of this paper, assume the transfer operator associated to the invariant circle has a spectrum that is two circles of radii $\rho_{-}<\rho_{+}<1$ (see section 2.2). The slow manifold is tangent to the spectral subbundle associated to the circle of radius $\rho_{+}$. The nonresonance conditions in [HdlL06b] in our simpler case just amount to $\rho_{+}^{i} \neq \rho_{-}$for all $i=2, \ldots, L$, with $L$ such that $\rho_{+}^{L+1} \rho_{-}<1$.

If we use only a first order approximation of the whisker, the domain where it is a good approximation can be very small and the globalization is harder. If we are computing the fast manifold, the iteration backward aligns the point with the fast manifold and reduces the error, so that it is not so crucial. On the other hand, iterating backward makes the slow manifold unstable. Hence, to obtain global slow manifolds, it is important to obtain high accuracy which can be best obtained by computing the expansion to a rather high order.

2.2. Analysis of the dynamics of linearization. In the analysis of an invariant torus, it is quite natural to study the dynamics of the linearization. As was discussed in great generality in [HdlL06b], the study of the linearization is very closely related to the properties of the linearization of the invariance equations. It is important to realize that the invariance equations (3) are functional equations and that therefore one should think of them as equations in a Banach space. One of the features of normal hyperbolicity theory is that there is a close 
connection between the geometric properties of the variational equations and the functional analysis properties of (3). This connection was realized in [Mat68, Sac78]. A treatment tailored for the examples appearing in this paper is in [HdlL03b].

At the geometric level, we observe that the linearized dynamics is given by

$$
\begin{aligned}
& \bar{v}=M(\theta) v, \\
& \bar{\theta}=\theta+\omega,
\end{aligned}
$$

where $v=\left(v_{x}, v_{y}\right) \in \mathbb{R}^{2}$, and $M(\theta)$ a $2 \times 2$ periodic matrix. In our applications, $M(\theta)=$ $\mathrm{D} F(K(\theta), \theta)$.

We use the notation

$$
\begin{aligned}
M(\theta, m) & =M(\theta+(m-1) \omega) \ldots M(\theta), \\
M(\theta,-m) & =M(\theta-m \omega)^{-1} \ldots M(\theta-\omega)^{-1}
\end{aligned}
$$

for $m>0$ and $M(\theta, 0)=\mathrm{Id}$.

On the functional analysis side, the cocycle (14) induces a transfer operator $\mathcal{M}_{\omega}$ defined in (13). We see that inverting $\mathcal{M}_{\omega}-\mathrm{Id}$ is precisely solving the Newton step in (4). Hence, it is quite important for the algorithms to decide whether 1 is in the spectrum or not.

In the subsequent sections, we will develop certain numerical observables that are relevant for the analysis of the invariant torus and which are readily computable and which have an influence on the behavior of the linearized dynamics and on the solvability of the functional equations for the parameterizations we are interested in.

Other issues, such as reducibility of the cocycle, have been discussed above (see also [Jor01, HdlL05a]).

In section 2.2.5, we will discuss the relation between the geometric properties and the spectrum of the linearization of the functional equations we are interested in.

2.2.1. Projectivization of the cocycle. To describe the $1 \mathrm{D}$ linear subspaces we are considering, it is natural to give a set of directions at every point. That is, to parameterize an invariant bundle, we give a mapping from the torus to the $1 \mathrm{D}$ projective space $\mathbb{P}^{1}$ which we represent as an angle in $[0, \pi)$ with $0, \pi$ identified.

Corresponding to the linear transformation (14), we can consider its action on directions given by the "projective cocycle"

$$
\begin{aligned}
\bar{\alpha} & =m(\alpha, \theta), \\
\bar{\theta} & =\theta+\omega,
\end{aligned}
$$

where $\bar{\alpha}=m(\alpha, \theta)$ is computed as follows:

- Given $\alpha \in[0, \pi[$, we consider

$$
v=\left(\begin{array}{c}
\cos \alpha \\
\sin \alpha
\end{array}\right)
$$

- We apply the cocycle, to obtain

$$
\bar{v}=M(\theta) v=|M(\theta) v|\left(\begin{array}{c}
\cos \tilde{\alpha} \\
\sin \tilde{\alpha}
\end{array}\right),
$$


where $\tilde{\alpha} \in[0,2 \pi[$ is an angle in the unit circle $(0=2 \pi)$.

- Finally, if $\tilde{\alpha} \in[0, \pi[$, we define $\bar{\alpha}=\tilde{\alpha}$, and if $\tilde{\alpha} \in[\pi, 2 \pi$ [, we define $\bar{\alpha}=\tilde{\alpha}-\pi$.

Notice that a subbundle in $\mathbb{R}^{2} \times \mathbb{T}$ is projectivized into a graph of a function $\theta \rightarrow \alpha(\theta)$ in $\mathbb{P}^{1} \times \mathbb{T}$. It is also very easy from this graph to know if the subbundle is orientable or not, and what is its index. The index of a subbundle $E$ is a semi-integer number $\frac{k}{2}$ that gives the number of turns that gives the fiber $E_{\theta}$ when $\theta$ goes from 0 to 1 . If $k$ is even, the subbundle is orientable, and if $k$ is odd, it is nonorientable. Doubling the period of $\theta$ is equivalent to taking a double cover so that we can orientate any 1D bundle.

In the case that the linear cocycle (14) has an exponential dichotomy, the projective cocycle (15) will have an attractor and a repellor. The attractor will be a representation of the unstable bundle (in the case of a hyperbolic torus) or the slow bundle (in the case of an attractive torus). We denote this bundle $E^{+}$. The repellor (i.e., the attractor under inverse iteration) will correspond to the stable bundle (in the case of a hyperbolic torus) or the fast bundle (in the case of an attractive torus). We denote this bundle $E^{-}$.

Remark 2.8. Note that the only bundles we are considering are continuous bundles. According to Oseledec's theorem [Ose68, Pes77], it could be natural to consider also measurable subbundles, especially in situations where there is bundle collapse (see section 6).

Nevertheless, we note that the motion on the circle admits only one invariant measure (the usual Lebesgue measure). Therefore, Oseledec's theorem produces only one set of Lyapunov multipliers and the Oseledec bundles can only be discontinuous in a set of measure zero (if this set is not empty, it is dense). It could happen that this is what is observed in section 6 .

The theory of reduction in the measurable category for $2 \mathrm{D}$ cocycles is studied in [Thi97].

2.2.2. Lyapunov multipliers. The maximal and minimal Lyapunov multipliers $\lambda_{+}$and $\lambda_{-}$are computed by taking a random vector $v \in \mathbb{R}^{2}$ and a random angle $\theta \in \mathbb{T}$ and applying the formula

$$
\lambda_{+}=\lim _{m \rightarrow \infty}|M(\theta, m) v|^{\frac{1}{m}}, \lambda_{-}=\lim _{m \rightarrow \infty}|M(\theta,-m) v|^{\frac{1}{-m}}
$$

To avoid the growth of the vectors, we do, as usual, scalings at each step. To compute the accuracy, we just compute the Lyapunov multipliers at the convergents of the continued fraction of $\omega$ and check that there is no appreciable change. An estimate of the error is the difference of two consecutive estimates.

We note that when the bundles are $1 \mathrm{D}$, we have that $\gamma(\theta, m)=\prod_{i=0}^{m-1} \gamma(\theta+i \omega)$, where $\gamma(\theta)$ is the - scalar - block of the matrix $M$ in the invariant bundle. We have that the corresponding Lyapunov multiplier is given by

$$
\begin{aligned}
\lambda & =\lim _{m \rightarrow \infty} \exp \left[\frac{1}{m} \sum_{i=0}^{m-1} \log |\gamma(\theta+i \omega)|\right] \\
& =\exp \left[\int_{0}^{1} \log (|\gamma(\theta)|) d \theta\right] .
\end{aligned}
$$

Furthermore, since in many cases the system is analytic, then the $1 \mathrm{D}$ bundles are also analytic, and the exponents for finite $m$ converge to their limit (16) faster than any power in 
$m$. (Of course, as the analyticity properties become weaker, the exponential convergence may take longer to manifest itself.)

The exponential convergence of the Lyapunov multipliers to their limiting value in the uniformly hyperbolic case can be used to speed the calculation or increase the accuracy.

One could consider also the use of quadrature algorithms to evaluate (16) (this is useful to make checks).

2.2.3. Separation of the bundles. An observable that will play an important role in our considerations (see, in particular, section 6) is the distance between two bundles in a exponential dichotomy. We will consider

$$
\Delta=\min _{\theta} \operatorname{dist}\left(E_{\theta}^{+}, E_{\theta}^{-}\right)
$$

this is the minimum angle between the bundles $E^{ \pm}$.

As is well known, the distance between the stable and unstable bundles plays a very important role in hyperbolicity theory. See [Fen72, HPS77]. The fact that to measure hyperbolicity one cannot just use the Lyapunov multipliers but has to use also the distance of the splittings is emphasized in [CLO0].

2.2.4. Rotation numbers. When considering normally elliptic tori in Hamiltonian systems, it will be useful to develop fast diagnostics that detect empirically the lack of reducibility. Besides the Lyapunov multipliers and the distance between invariant bundles, the observables that we will use are roughly patterned on the rotation number which measures the averaged amount of rotation per turn.

We note that, when the cocycle is reducible, the cocycle is a rotation modulo a change of coordinates. Therefore, there are many ways to compute the rotation number. There are several definitions of rotations numbers in the mathematical literature. See [Rue85, NNO98, FJN03a, FJN03b] for a review.

Unfortunately, the average amount of rotation requires us to consider the angles not modulo $2 \pi$ but rather on a lift of the circle. When iterating maps, the information needed to lift the angles seems to involve global considerations (e.g., choosing an origin for the angles and propagating this choice along the circle, e.g., by interpolating the map by a globally defined family). We have not succeeded in implementing any of these rigorous definitions in a numerically efficient manner because the global considerations required by the definitions are hard to implement efficiently.

Since our purpose is just to get some rough understanding of reducibility, we have used several diagnostics that work well in the reducible case. When they start to show changes or erratic behavior, this is a sign that reducibility has broken down. The thresholds computed in this way can subsequently be refined.

The first method is to compute the rotation number $\gamma$ by the formula

$$
\gamma_{a}=\lim _{m \rightarrow \infty} \frac{1}{m} \sum_{i=0}^{m-1} \operatorname{ang}(M(\theta, i) v, M(\theta, i+1) v),
$$

where $\operatorname{ang}(v, w)$ denotes the oriented angle from $v$ to $w$, from $-\pi$ to $\pi$. 
An easy way to compute the Sturmian rotation number inspired by [DS83b] (see also [FJN03a]) is defined as follows. If $v^{i}=\left(v_{x}^{i}, v_{y}^{i}\right)$ denotes $M(\theta, i) v$, for $m>0$ we define $N(m)$ as the number of times in which $v_{y}^{i}$ changes sign for $i=0 \div m$. Then we define the rotation number as

$$
\gamma_{s}= \pm \pi \lim _{m \rightarrow \infty} \frac{N(m)}{m}
$$

In order to specify a sign for the rotation number, we can see if, "on average," the vector spins counterclockwise $($ sign +1$)$ or clockwise $(\operatorname{sign}-1)$.

We note that both definitions, even if they make sense for differential equations or small steps, may run into trouble in some cases. For example, if we were taking long steps in a differential equation, the angle could rotate by more than a turn. Similarly, the Sturmian values could miss several changes of sign. In the reducible case, these mistakes happen in a very systematic way. On the other hand, in the nonreducible case, it is not possible to say that they will occur with a fixed frequency. For us, the above observables are just quick diagnostics for resonances and lack of reducibility.

2.2.5. Spectral properties of the transfer operator. The transfer operators that appear in our case are very special for several reasons: The motion in the base is a rotation and the nontrivial bundles are 1D; hence the restriction of the derivative is commutative.

One of the consequences of the theory developed in [HdlL03b] is that the spectrum is largely independent of the spaces considered so that we will not specify the space we are considering the transfer operator acting on.

Putting together results from [HdlL03b], we obtain that there are only a few possibilities for the spectrum of the transfer operator $\mathcal{M}_{\omega}$ introduced in (13):

(a) Two circles of radii $\rho_{-}<\rho_{+}$. In this case, we have a decomposition into continuous invariant bundles $\mathbb{R}^{2}=E_{\theta}^{+} \oplus E_{\theta}^{-}$for all $\theta \in \mathbb{T}$, characterized by the uniform rates of growth

$$
\begin{aligned}
& v \in E_{\theta}^{-} \quad \Leftrightarrow \quad|M(\theta, m) v| \leq C\left(\rho_{-}+\delta\right)^{+m}|v|, m \geq 0, \\
& v \in E_{\theta}^{+} \quad \Leftrightarrow \quad|M(\theta,-m) v| \leq C\left(\rho_{+}-\delta\right)^{-m}|v|, m \geq 0,
\end{aligned}
$$

where $\delta$ is small enough, and the constant $C$ is uniform (it does not depend on $\theta$ or $v)$. The vectors in $E_{\theta}^{ \pm}$have a Lyapunov multiplier equal to $\lambda_{ \pm}=\rho_{ \pm}$. We say that the cocycle $M$ has an exponential dichotomy. In particular, if $\rho_{-}<1<\rho_{+}$, the cocycle is uniformly hyperbolic and of saddle type, and $E^{-}$and $E^{+}$are the stable and the unstable subbundles; if $\rho_{-}<\rho_{+}<1$, the cocycle is uniformly hyperbolic and of attracting node type, and $E^{+}$and $E^{-}$are the fast stable and the slow stable subbundles.

The subbundles $E_{\theta}^{ \pm}$are as smooth as $M$. Moreover, if the external rotation is Diophantine, the dynamics on each bundle can be reduced to a multiplication by a constant [JS81].

(b) One circle of radius $\rho$. All the vectors have Lyapunov multipliers equal to $\rho$. This case appears naturally in the elliptic case in Hamiltonian systems, with $\rho=1$. 
(c) One annulus of radii $\rho_{-}<\rho_{+}$. In this case, there is no continuous invariant splitting but, according to [Ose68, Pes77], there is a measurable splitting characterized by the fact that the corresponding Lyapunov multipliers are $\lambda_{ \pm}=\rho_{ \pm}$(see [CL99] and the references therein). If $\rho_{-}<1<\rho_{+}$, the cocycle is nonuniformly hyperbolic.

The forward and backward Lyapunov multipliers of vectors lie between $\rho_{-}$and $\rho_{+}$ [Sac78, HdlL03a], but we emphasize that the maximal and minimal Lyapunov multipliers $\lambda_{ \pm}=\rho_{ \pm}$are attained in a set of full Lebesgue measure.

Moreover, for all $\rho \in\left[\rho_{1}, \rho_{2}\right]$ there is a vector $v \in \mathbb{R}^{2}$ and an angle $\theta \in \mathbb{T}$ whose scaled orbit $\left\{\frac{1}{\rho^{m}} M(\theta, m) v\right\}_{m \in \mathbb{Z}}$ is bounded [Mn78, CL99, HdlL03a].

Remark 2.9. If a cocycle is reducible (or almost reducible), then the spectrum of the transfer operator is one of the alternatives (a) and (b) above [HdlL03b]; that is, the spectrum is either two circles or one circle.

Almost conversely, in our case, when we are in case (a), the cocycle will be reducible.

2.2.6. Rotating transformations. Assume the cocycle (14) is reducible to a matrix $\Lambda=$ $\rho R_{\gamma}$, with $R_{\gamma}$ being a rotation matrix of angle $\gamma$. This is that there exists a periodic matrix $P(\theta)$, of period 1 (or 2 ), such that

$$
M(\theta) P(\theta)=\rho P(\theta+\omega) R_{\gamma} .
$$

For $k \in \mathbb{Z}$, consider the rotating matrix $R_{k}(\theta)=R_{\pi k \theta}$. For $k$ even, this is of period 1 , but for $k$ odd, this is of period 2. Then, it is easy to see that the matrix $P_{k}(\theta)=P(\theta) R_{k}(\theta)$ satisfies

$$
M(\theta) P_{k}(\theta)=\rho P_{k}(\theta+\omega) R_{\gamma-\pi k \omega}
$$

So, the cocycle is also reducible to $\Lambda_{k}=\rho R_{\gamma-\pi k \omega}$.

This freedom in choosing the reduced matrix is very useful in numerical computations. For instance, assume that the torus has a cocycle that is reduced to a matrix $\rho R_{\gamma}$. When perturbing the system, the rotation number $\gamma$ crosses resonances. Assume we are near a resonance $k_{1}: k_{2}$, that is,

$$
\left|\frac{\gamma}{2 \pi}-\frac{k_{1}+k_{2} \omega}{2}\right| \simeq 0
$$

Then, the rotating transformation $R_{k_{2}}$ lets us reduce the system to the matrix

$$
\Lambda_{k_{2}}=\rho R_{\gamma-\pi k_{2} \omega} \simeq \rho R_{k_{1} \pi}
$$

So, the matrix $\Lambda_{k_{2}}$ is close to a diagonal matrix with the same entries in the diagonal.

It is obvious that $k_{1}$ gives the sign of the elements of the diagonal. The number $k_{2}$ is related to the topology (the index) of the dynamics around the torus. The transformation $R_{k_{2}}$ straightens the dynamics of the cocycle but keeps the topological information. The index of $R_{k_{2}}$ is $\frac{k_{2}}{2}$. We will clarify these ideas with some examples later. 
2.3. Computer programs. The algorithms have been implemented in the $\mathrm{C}$ language and have been run under the Linux environment. We have applied them to compute invariant manifolds for 2D quasi-periodic maps, with one external frequency.

The algorithms have been applied to quasi-periodic perturbations of the Hénon map and the standard map (although with slight modifications they can be applied to other examples). In the examples the system is coupled with a quasi-periodic external perturbation via a parameter $\varepsilon$.

With this set of programs we can do the following:

1. Compute invariant and periodic circles, with continuation with respect to the parameter $\varepsilon$. The input is the set of parameters of the autonomous system ( $a$ and $b$ in the case of the Hénon map, and $K$ in the case of the standard map), the period with some specification of the initial periodic point (in the case of the standard map, for instance, that is its rotation number and some symmetry), and a list of $\varepsilon$ 's; the output is a set of files containing the Fourier expansions of the circles for these $\varepsilon$ 's. Other programs let us obtain meshes of the circles to make plots. In the implementation of the projection method and the reducibility method, the programs include in the output files the reducing transformations and the reduced matrices.

2. Compute whiskers of invariant and periodic circles: The input is one of the outputs of the previous programs, that is, a file containing the parameters and the Fourier expansions giving the circle; the output is, in the case that the circle is hyperbolic, the files containing the Fourier-Taylor expansions of the whiskers. If in the input file there are the reducing frames (because we have used the projection method or the reducibility method), these programs use them to do the computations. If not, the programs implement the power method to compute the bundles, and we refine the computation using reducibility [HdlL05a] (we have also done tests and computations by discretizing the transfer operator and computing the eigenvalues of the discretization [Jor01]). These bundles are the frames where the whiskers are constructed.

3. Globalize the whiskers: A first program computes the intersection of a whisker with a given plane $\theta=\theta_{0}$. A second program computes a $2 \mathrm{D}$ grid of points of the surface, by doing several sections of the object with equidistant time planes. In both cases we have to specify the total arc-length of the sections, the maximum distance between consecutive points in the curves, and the local error to compute the fundamental domain. This is very standard (see, for instance, [KO98]).

4. Once we have computed the meshes and grids of the objects, we can plot them using standard packages. To produce the figures of this paper we have used xmgr, gnuplot, and MATLAB.

We have also written many other test programs - for example, computation of Lyapunov multipliers and rotation numbers of a cocycle, computation of attractor and repellor of a projective cocycle, and power method.

We have also written the versions of the programs to work in the double cover. This is just substituting the system (2) by

$$
\left(\begin{array}{c}
z \\
\theta
\end{array}\right) \longrightarrow\left(\begin{array}{c}
F_{\varepsilon}(z, 2 \theta) \\
\theta+\frac{\omega}{2}
\end{array}\right)
$$


This trick lets us work with 1-periodic functions and avoid 2-periodic functions.

The timings reported correspond to work in different machines that we will specify. For instance, some results have been produced with a desktop computer with a $700 \mathrm{MHz}$ processor. From this, the reader can guess timings in other machines.

In the following we will discuss some efficiency properties of the implementation in some representative examples and, much more importantly, we will present some conjectures that we have obtained by observing phenomena that are not yet justified by a full mathematical theory. We hope that reporting these conjectures will stimulate mathematical work.

3. Example 1: The rotating Hénon map. The first example we consider is a quasiperiodic perturbation of the Hénon map. The rotating Hénon map is the quasi-periodic dissipative map on the plane given by

$$
\begin{aligned}
& \bar{x}=1+y-a x^{2}+\varepsilon \cos (2 \pi \theta), \\
& \bar{y}=b x, \\
& \bar{\theta}=\theta+\omega \quad(\bmod 1),
\end{aligned}
$$

where $a, b$ are the parameters of the Hénon map and $\varepsilon$ leads to a quasi-periodic forcing. The motion of the angle variable $\theta$ is a rotation by the irrational number $\omega$. We have taken the golden mean $\omega=\frac{1}{2}(\sqrt{5}-1)$.

For $\varepsilon=0$, and we suppress the angle variable, we have the classical Hénon map

$$
\begin{aligned}
& \bar{x}=1+y-a x^{2}, \\
& \bar{y}=b x .
\end{aligned}
$$

This map has two fixed points

$$
p_{ \pm}=\left(x_{ \pm}=\frac{b-1 \pm \sqrt{(b-1)^{2}+4 a}}{2 a}, y_{ \pm}=b x_{ \pm}\right)
$$

provided that $(b-1)^{2}+4 a>0$ (for instance, if $a>0$ ). There exists also a 2-periodic orbit, provided that $4 a-3(1-b)^{2}>0$ (at $a=\frac{3}{4}(1-b)^{2}$ there is a period doubling bifurcation of $\left.p_{+}\right)$. Both points of the 2-periodic orbit correspond to the solutions of the quadratic equation

$$
a^{2} x^{2}-a(1-b) x-a+(1-b)^{2}=0 .
$$

These fixed points and periodic orbits turn into invariant curves and periodic curves under the quasi-periodic perturbation. (See Figure 1.)

The map (25) was studied in [SFKA96] as an example of system with a strange nonchaotic attractor (SNA). In [OF00] the structure of this attractor was studied by means of computation of stable and unstable manifolds of invariant curves of saddle type, based on the algorithms appearing in [KO98]. The computation of slow manifolds considered here does not seem to have been considered in the literature before.

In the following, we fix the parameters of the Hénon map to be $a=0.68$ and $b=0.1$. We have used several computer programs to continue invariant and periodic tori with respect to $\varepsilon$ and to compute their whiskers. 

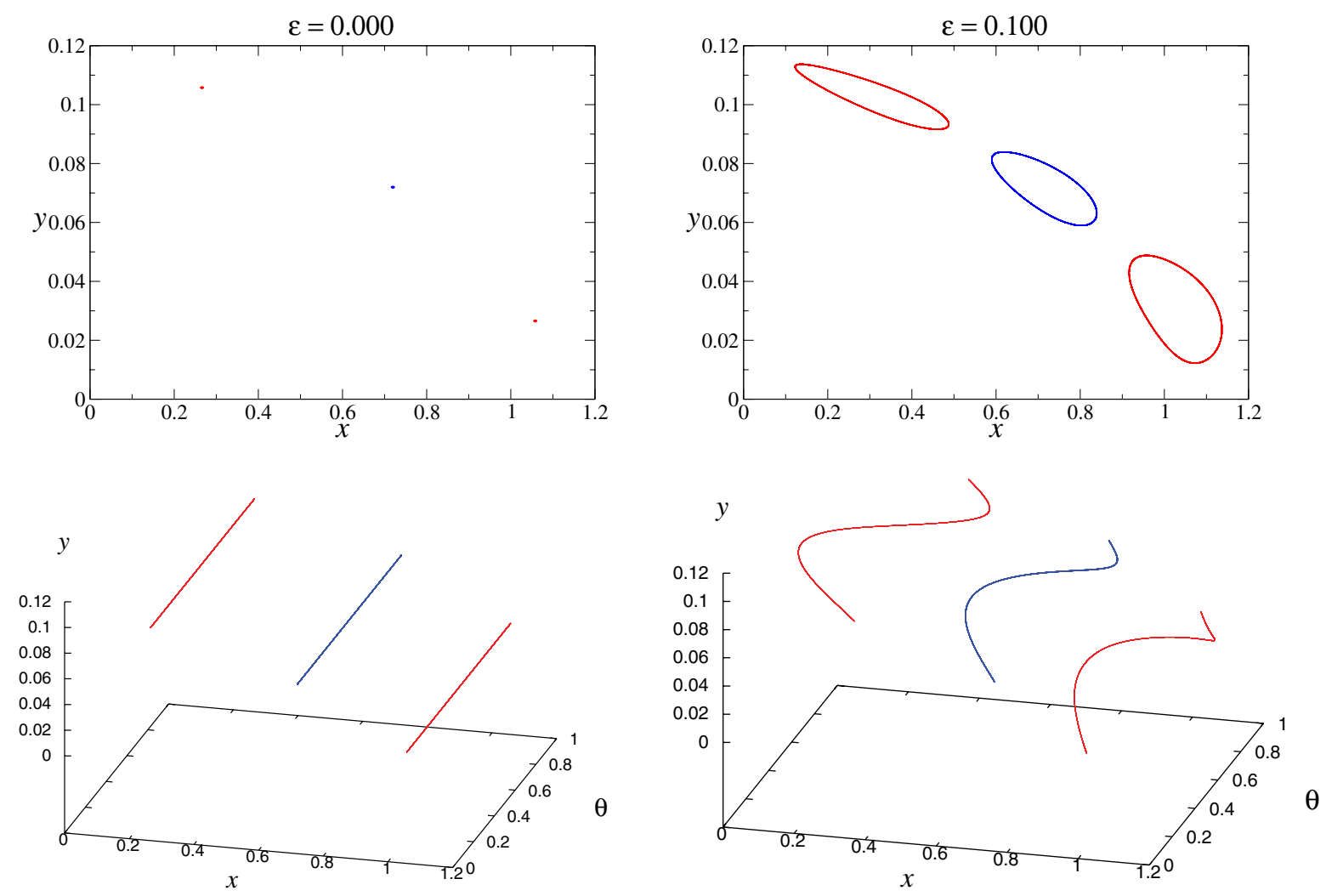

Figure 1. A saddle-type fixed point (blue) and an attracting 2 periodic point (red) of the Hénon map with $a=0.68$ and $b=0.1$ become closed curves when we add the external perturbation. The lower pictures are $3 D$ views in the extended phase space.

Starting from the fixed point $p_{+}$, the continuation of the invariant curve for $\varepsilon=0 ., 0.1$, $0.2,0.3,0.4,0.5$ is done in less than one second using 50 harmonics in the Fourier expansions and running on a Pentium III at $700 \mathrm{MHz}$. The errors in the functional equations are of the order $10^{-19}$. (See Figure 1 for some examples.)

For $\varepsilon=0.1$, the invariant curve is of saddle type. We can also compute the whiskers of this invariant curve up to order 30 (the degree of the Fourier-Taylor polynomials), in less than 8 seconds. If the linear approximations of the manifolds are not provided as an input, the program uses the power method to find them. We use these local expansions to globalize the manifolds. For instance, to compute points of the curve of intersection with the plane $\theta=0$ of one of the branches of the unstable manifold (length $=10$, distance between points $=0.01$, local error $=10^{-10}$ ) takes about 4 seconds. With this technology we can also produce 3D grids (taking essentially different sections).

Figure 2 shows the section with the plane $\{\theta=0\}$ of some invariant curves and their whiskers for $\varepsilon=0.1$.

3.1. Continuation of an invariant torus: A brief description. In the following sections we will explain the bifurcations and transitions we have encountered in continuing the invariant 


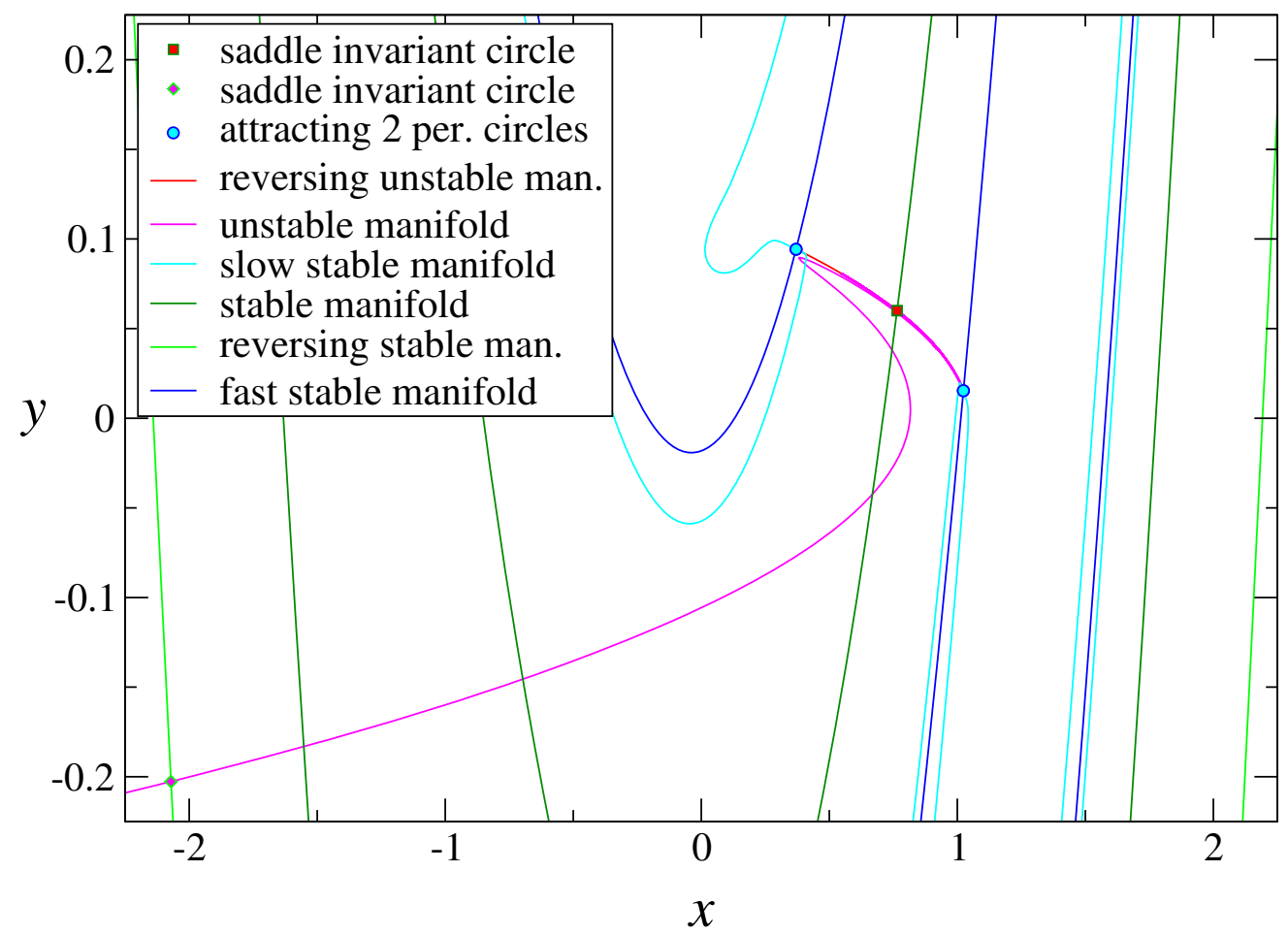

Figure 2. Sections with the plane $\theta=0$ of invariant $2 D$ manifolds associated to invariant curves. The parameters of the rotating Henón map are $a=0.68, b=0.1$, and $\varepsilon=0.1$.

circle corresponding to the fixed point $p_{+}$of the Hénon map. In particular, in section 6 , we will report on a mechanism of loss of exponential dichotomy and breakdown.

In the following exploration, which is representative of many others, we have fixed $a=0.68$ and $b=0.1$ and have increased $\varepsilon$ from 0 . Figure 3 shows the continuation of the invariant torus, whose analysis is detailed below.

To measure the hyperbolicity around the torus, we have used two observables: the maximal Lyapunov multiplier $\Lambda=\lambda_{+}$(the minimal Lyapunov multiplier is $\lambda_{-}=|b| / \Lambda$ ) and the distance $\Delta$ between the attractor and repellor of the projective cocycle. The results are displayed in Figure 4, where we observe several transitions, labeled with the letters a, b , c, d, e.

The fixed point $p_{+}$of the Hénon map is of saddle type. When we take the Cartesian product with the external rotation, the fixed point becomes a "straight" invariant circle (see the first picture of Figure 3, where we also draw the 2-periodic attracting circle).

A bird's eye analysis of the bifurcations detected easily in Figure 4 is as follows.

(a) At $\varepsilon_{\mathrm{a}} \simeq 0.2549$, the spectrum of the linearization crosses 1 and -1 is an eigenvalue. The torus goes from being unstable to being stable, and there is a period doubling bifurcation [BHTB90]. The leading spectral bundle goes from being unstable to being slowly stable. 

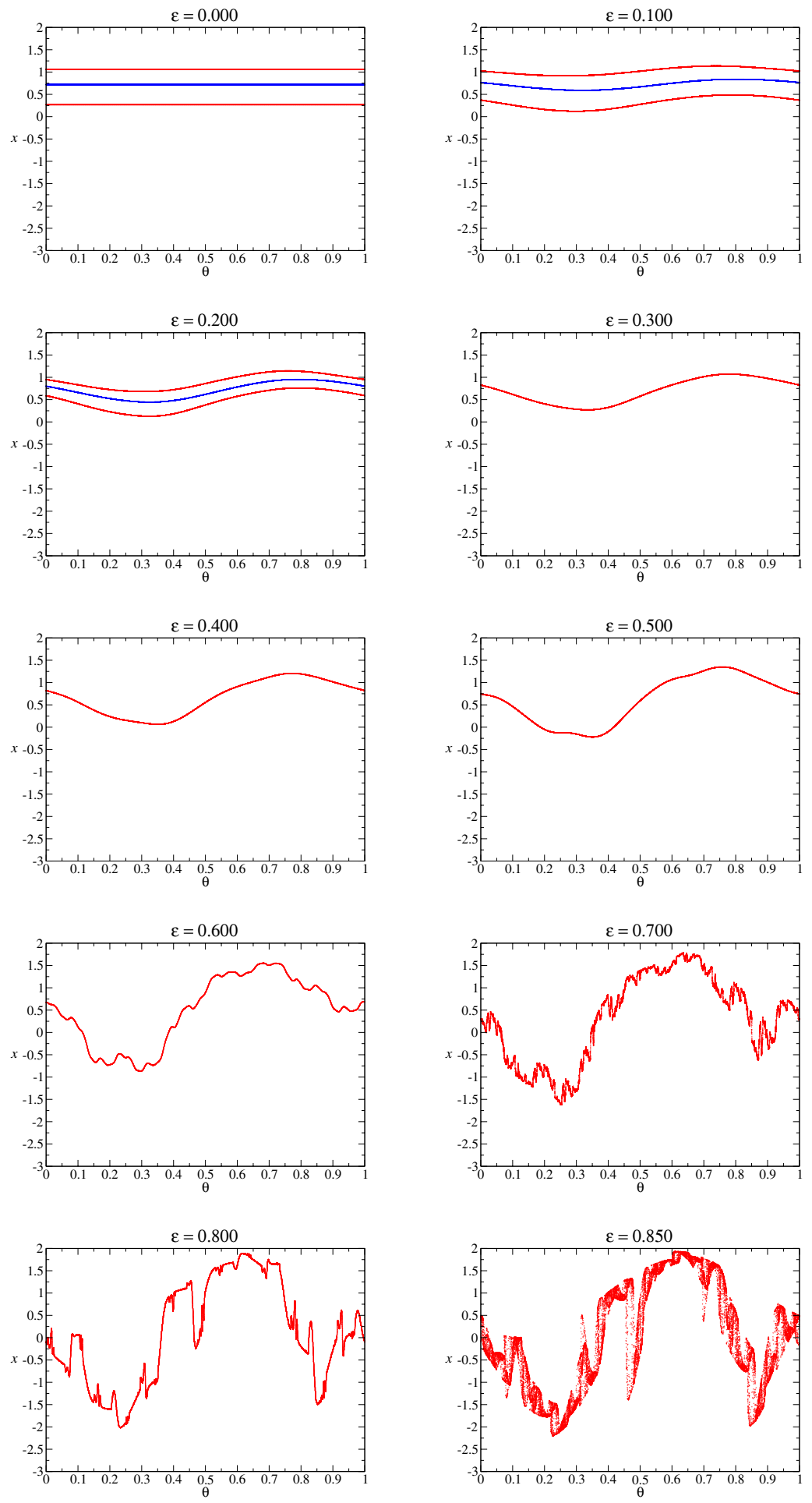

Figure 3. $\theta x$ projection of an attractor (red). 


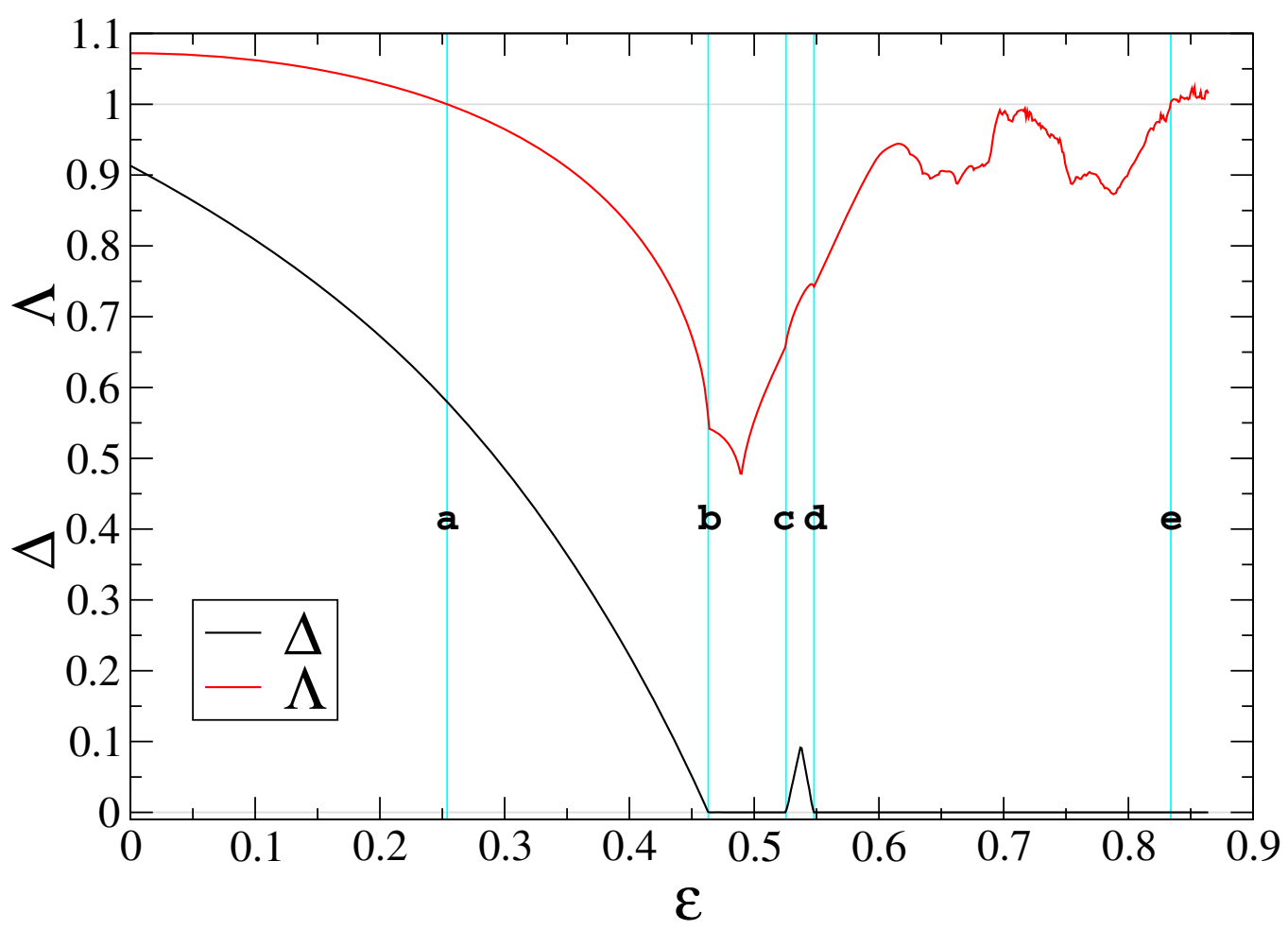

Figure 4. Distance $(\Delta)$ between the invariant bundles and Lyapunov multiplier $(\Lambda)$ of an invariant torus for the rotating Hénon map, continued with respect to $\varepsilon$.

(b) This is the first instance of a global bifurcation, which we call the bundle merging scenario. This bifurcation will be described in more detail in section 6 . In particular, we will identify quantitative regularities.

The spectrum remains two separate circles for $\varepsilon<\varepsilon_{\mathrm{b}} \simeq 0.4633$. Nevertheless, the separation between the bundles becomes smaller and seems to tend to zero as $\varepsilon$ approaches $\varepsilon_{\mathrm{b}}$ from below.

For $\varepsilon$ slightly bigger than $\varepsilon_{\mathrm{b}}$, it seems that there are no separate continuous invariant fast and slow bundles.

(c, d) The transition $\mathrm{c}$, which happens at $\varepsilon_{\mathrm{c}} \simeq 0.5256$, seems to be a reverse of the bundle merging scenario found in $\mathrm{b}$. The bifurcation in $\mathrm{d}$, which happens at $\varepsilon_{\mathrm{d}} \simeq 0.5475$, is a direct bundle merging bifurcation.

(e) At $\varepsilon_{\mathrm{e}} \simeq 0.8337$, the spectrum of the linearization touches 1 , and the torus seems to disappear completely. In contrast with the quasi-periodic saddle-node bifurcation, there does not seem to be any companion unstable circle nearby. For $\varepsilon$ slightly smaller than $\varepsilon_{\mathbf{e}}$ it seems that there are no continuous invariant bundles.

This transition seems hard to understand. We have not found any quantitative reg- 
ularity. We just make here the simple remark that this transition seems visually similar to the fractalization mechanism that has been described several times in the literature [Kan84, NK96, SFKA96] (see also the review [PNR01]). In [SFKA96] there is a study of this transition in the rotating Hénon map.

Moreover, it seems that the torus is continuous (even analytic [HdlL06b]) until the outer radius of the spectrum (the maximal Lyapunov multiplier) touches 1 [HdlL06a, HdlL05c]. Notice that a normally hyperbolic torus persists under a sufficiently small perturbation, so the torus could survive until the maximal Lyapunov multiplier crosses 1 and the torus loses its normal hyperbolicity.

We would also like to refer to [HS05], which shows that some of the conclusions before may have been based on numerics which are not accurate.

Remark 3.1. We emphasize that the above list of the bifurcations is not exhaustive. As we will see in section 6 , if we look very carefully in the interval $\left[\varepsilon_{\mathrm{b}}, \varepsilon_{\mathrm{c}}\right]$, there are other bundle merging bifurcations. Indeed, it seems that bundle merging bifurcations generate other "daughter" bundle merging bifurcations close by. See section 6.3.4.

The beginning of Figure 4 is computed using the reducibility method. This method can continue over the period doubling bifurcation. Nevertheless, it gets stuck on the bifurcations b, which correspond to lack of reducibility. To get an idea of the accuracy we display some typical results in Table 1.

We note that in this case, the fact that the reducibility fails does not imply that the torus cannot be continued. Since the torus is attractive, either the full matrix Newton method or even direct iteration can be used to compute the torus accurately.

Note that in the intervals $\varepsilon_{\mathrm{b}} \leq \varepsilon \leq \varepsilon_{\mathrm{c}}$ and $\varepsilon_{\mathrm{d}} \leq \varepsilon \leq \varepsilon_{\mathrm{e}}$ it seems that there are no continuous invariant subbundles and that the spectrum is not reducible (it is a full annulus). Indeed, it seems empirically that this lack of reducibility is abundant; that is, nonreducibility holds in a set of parameters of positive measure. Moreover, if we change slightly the other two parameters ( $a$ and $b$ ), the same phenomena is observed.

There are mathematical results showing that reducibility is dense for cocycles taking values in $S U(2)$ or in other compact groups [Kri99c, Kri99b, Kri99a, Kri01]. Some recent results for noncompact groups are in [AK03].

These mathematical results suggest that the regions where reducibility fails have a complicated structure. If we perform a numerical experiment with a prescribed accuracy, we obtain a partition of parameter space in intervals where the finite precision criterion for reducibility applies or not. In the cases where the criterion applies, the validating results in [HdlL06b] show that reducibility is indeed true. In the cases where the finite precision criterion fails, however, it means only that we need more delicate exploration. A higher precision computation may reveal other intervals where the criterion for reducibility holds. In the intervals not covered by this refined criterion, we can, of course, try more refined observations, and so on. Some of these explorations motivated by the mathematical insights are reported in section 6.3.4.

3.2. Breakdown of exponential dichotomies. To study the mechanism of loss of reducibility, we study the projective cocycle associated to the torus (see (15) in section 2.2) around the critical value. We will see that the breakdown of reducibility is associated to the 

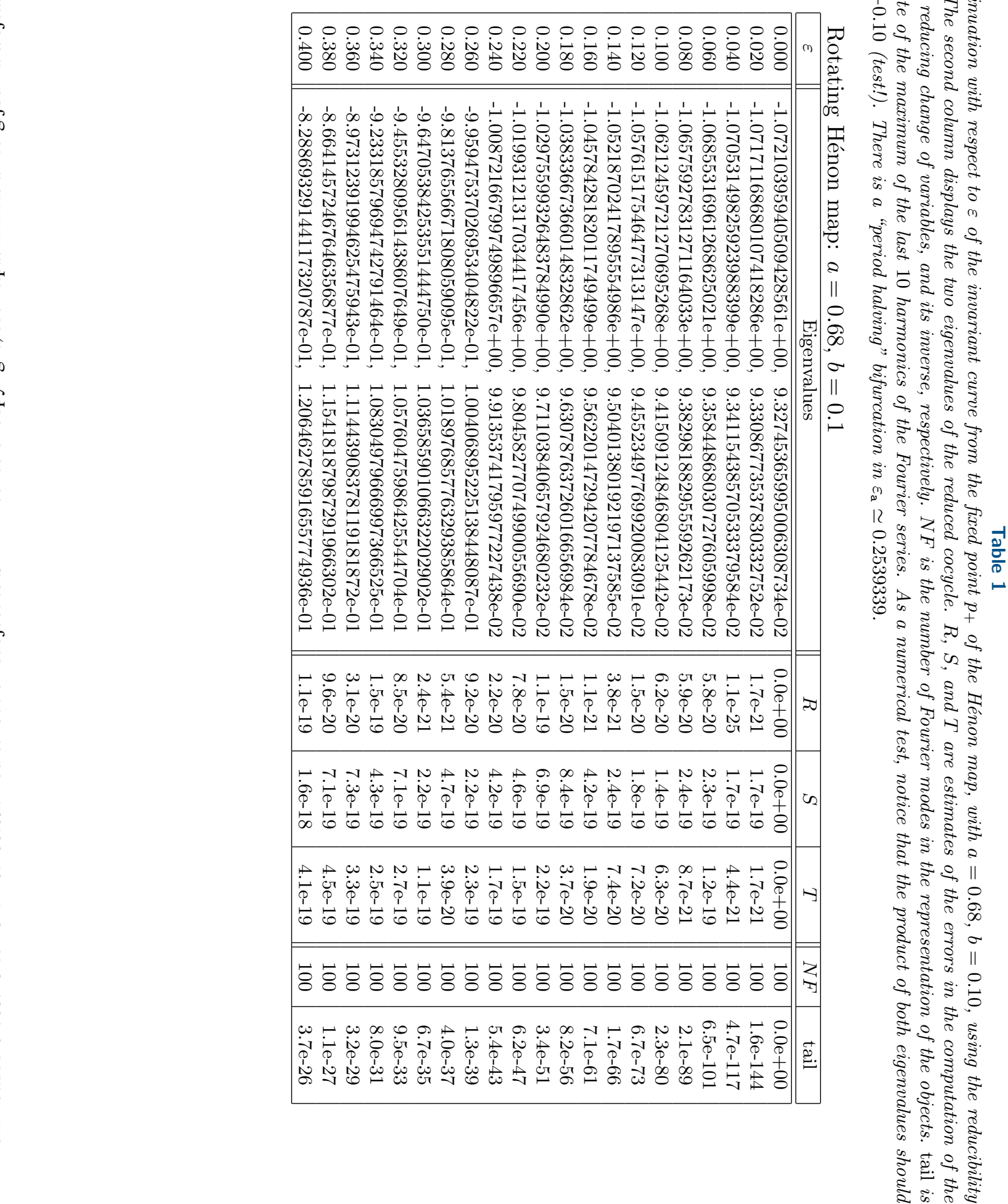

\%

1 क ह ह

11 芆

3.

요 ह

की

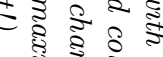

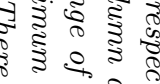

क.

$=$ क हैं

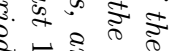

จ

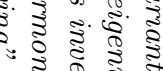

हैं की की है है

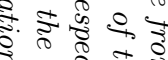

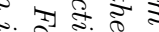

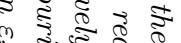

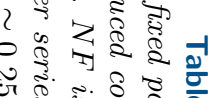

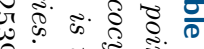

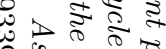

8
a

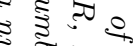

$3 \sin ^{3}$

3.

$+\sqrt{3})$

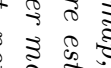

क.

ป ล.

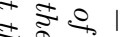

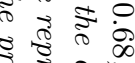

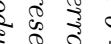

离

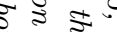

๑.

욜

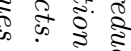

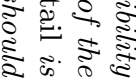


breakdown of the existence of exponential dichotomies. That is, the fast and slow bundles merge. See the discussion in section 2.2.5.

By direct iteration of (15), the orbits will converge to an attractor. Similarly, the iteration of the projectivization of the inverse cocycle will converge to a repellor. If there are slow and fast invariant bundles, the attractor corresponds to the slow bundle and the repellor to the fast bundle. The results of this experiment are depicted in Figure 5. Compare the results with those in Figure 4.

For $\varepsilon=0.400$ the attractor and repellor of the projective cocycle are smooth curves. These correspond to the invariant subbundles of the cocycle corresponding to the least and most attractive eigenvalues, respectively.

We note that, as $\varepsilon$ approaches a critical value $\varepsilon_{\mathrm{b}}$, the attractor and the repellor in the projective space approach each other. We note that the theory in [JS81, HdlL03b] ensures that, provided that the distance remains positive, the invariant bundles will be analytic.

In fact, the distance between both curves for $\varepsilon=0.463$ is of the order of $10^{-3}$. For $\varepsilon=0.463254$ the distance is of the order of $10^{-6}$. One can presume that the distance converges to zero as $\varepsilon$ converges to the critical value $\varepsilon_{\mathrm{b}}$. We have detected that the critical parameter is

$$
\varepsilon_{\mathrm{b}} \simeq 0.4632544711720
$$

and that the corresponding maximal Lyapunov multiplier (the spectral radius of the transfer operator) is $\Lambda_{\mathrm{b}} \simeq 0.542306556$. Figure 6 depicts more accurately the attractors and repellors for the projective cocycle at the presumed critical value.

In section 6 we will present some quantitative regularities of the phenomenon, which we have found empirically, as well as other phenomena.

3.3. Topological evidence of bundle collapse (I). An indirect proof that there is a bundle collapse is the following.

Notice that when the cocycle has two continuous invariant bundles, it is reducible to a constant diagonal matrix, since the external rotation is Diophantine [JS81].

In the continuation of the attracting torus, we observe that before the transition $\mathrm{b}$, the leading eigenvalue is negative and the other is positive. In particular, we have the following.

- For $\varepsilon=0.463$, the torus is attracting and the cocycle is reducible to a constant diagonal matrix

$$
\operatorname{diag}(-0.603430499529439903989,0.165719167456701022933) \text {. }
$$

After the transition c reducibility is recovered, but the leading eigenvalue is positive. In particular, we have the following.

- For $\varepsilon=0.530$, the torus is attracting and the cocycle is reducible to a constant diagonal matrix

$$
\operatorname{diag}(0.694546750046480781363,-0.143978789035162504966) .
$$

We emphasize that the calculations mentioned above are very reliable since reducibility methods apply and the results can be validated using the methods in [HdlL06b]. Hence, we 

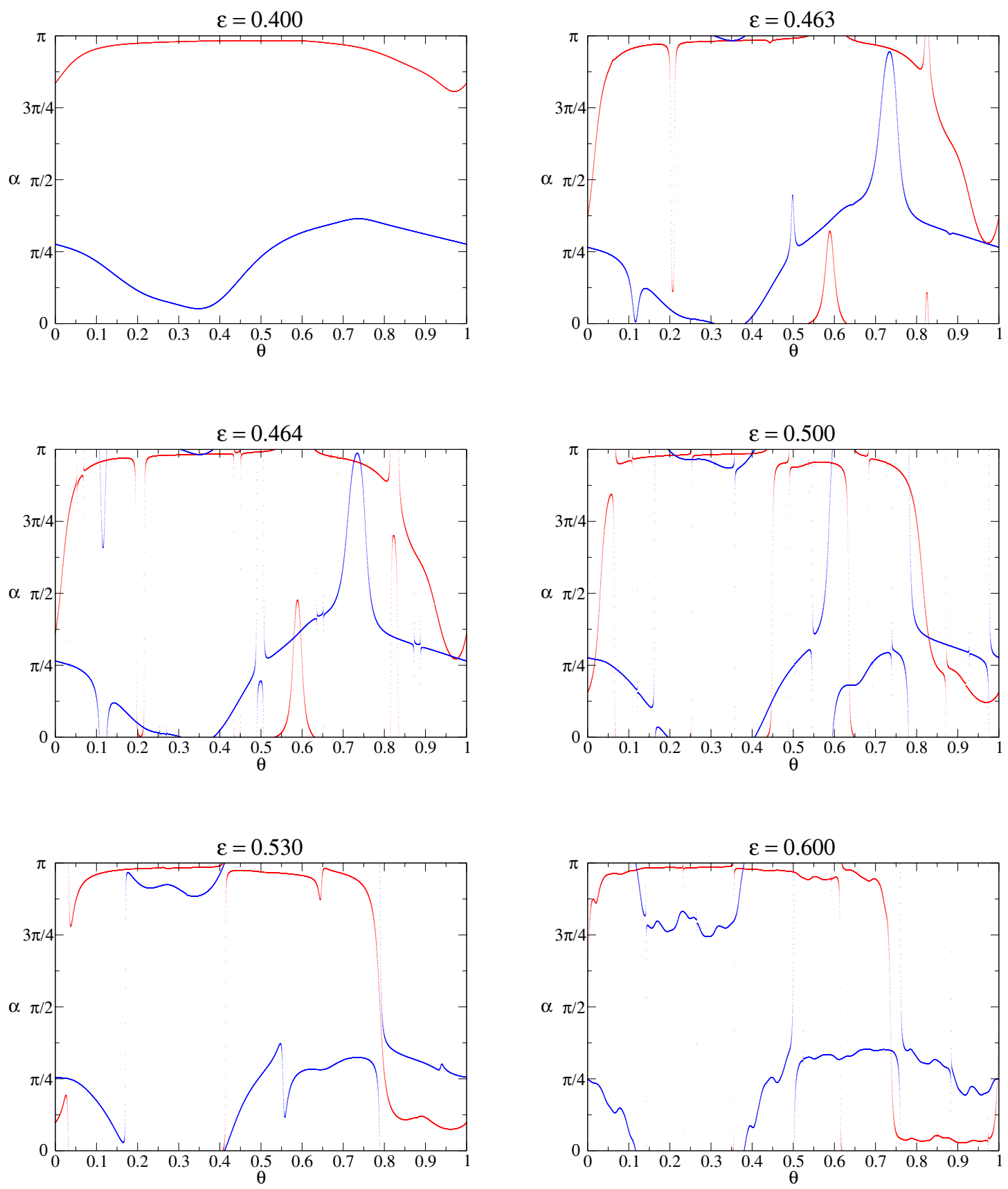

Figure 5. Attractor (red) and repellor (blue) of the projective cocycle of the attracting invariant curve for the Hénon map.

are quite sure that from $\mathrm{b}$ to $\mathrm{c}$ in Figure 4 there is a change of topology/dynamics of the invariant bundles. 

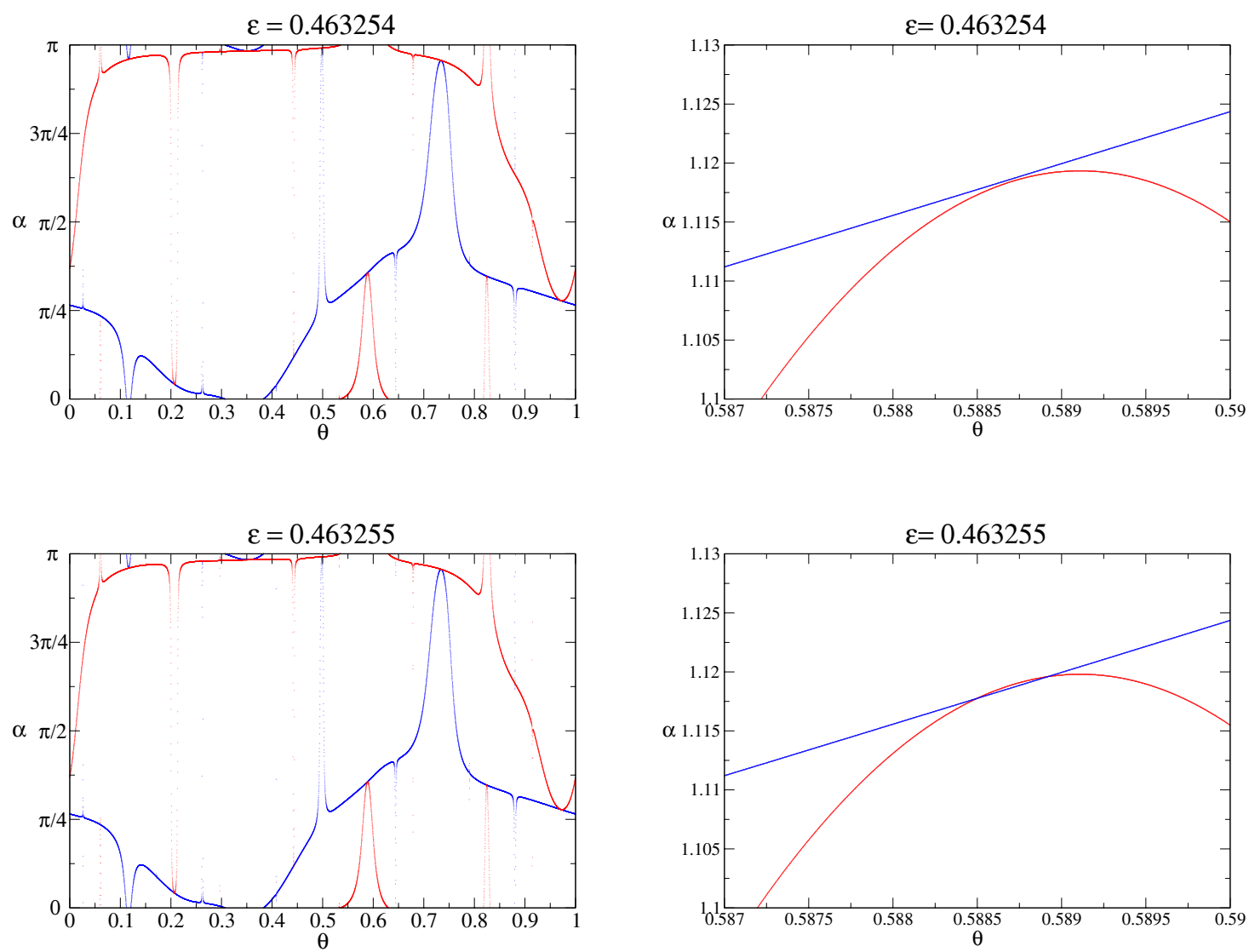

Figure 6. Slow (red) and fast (blue) directions before and after the transition of the lack of reducibility, and the magnification of a very small region. The distance between the attractor and the repellor for $\varepsilon=0.463254$ is $\Delta \simeq 1.86 \cdot 10^{-6}$.

We also observe that the maximal and minimal Lyapunov multipliers are different during the continuation. If they were equal, they would be equal to $\sqrt{|b|} \simeq 0.3162$, which is not the case (see Figure 1).

As a result, the torus cannot remain reducible during the whole continuation, but the Lyapunov exponents cannot become close. The only possibility is that at some parameter value the torus becomes nonreducible (hence there are no $1 \mathrm{D}$ invariant bundles) even if the Lyapunov multipliers remain different.

Remark 3.2. Note that the argument above can only hope to show that there is a point in the interval where reducibility fails but nevertheless the spectrum is not trivial. Topological methods cannot hope to show that the transition is sharp-indeed, we do not expect it is based on our numerics - and much less the quantitative regularities discussed in section 6.2.

We think that the argument above can be made rigorous. The only ingredients that we need are that the index changes at the end of the interval and that the Lyapunov exponents do not degenerate. 
Note that, given the validation results in [HdlL06b], to validate the results on the index, all that we need to do is show that the invariance equations for the torus and for the bundles are satisfied with enough accuracy that we can assess the sign of the averaged multipliers. The calculations we have performed can routinely be transformed into interval calculations [KSW96] and we certainly expect that the precision will be enough.

Since the Jacobian of the transformation is constant, the fact that the Lyapunov exponents do not coincide can be established if we prove lower bounds for one of the Lyapunov exponents. These lower bounds can be achieved by applying the method of [Her83] to the finite approximation computed.

We certainly hope that the present detailed numerics inspire computer assisted proofs and, of course, more rigorous studies.

Remark 3.3. The arguments above to show that there is a value of the parameter without reducibility can be modified slightly to show that they do not rely on the fact that the rotation is Diophantine.

Just notice that a normalization of an orientable bundle in $\mathbb{S}^{1} \times \mathbb{T}$, instead of $\mathbb{P}^{1} \times \mathbb{T}$, produces two curves $\left(\mathbb{S}^{1}\right.$ is a double covering of $\mathbb{P}^{1}$ ). (If the bundle were nonorientable, we would also double the base torus $\mathbb{T}$.)

In the representation of the bundles in $\mathbb{S}^{1} \times \mathbb{T}$, for $\varepsilon=0.463$ the slow bundle corresponds to a 2-periodic curve, and the fast bundle corresponds to two fixed curves. Moreover, for $\varepsilon=$ 0.530, the slow bundle corresponds to two fixed curves, and the fast bundle corresponds to a 2periodic curve. These two cases are homotopically nonequivalent. Hence, it is impossible that the torus remains reducible throughout the continuation. We also note that this breakdown in reducibility has to happen while the Lyapunov multipliers remain different.

Hence, the mechanism of bundle collapse is independent of the Diophantine properties of the rotation. The above version of the argument applies to Liouville numbers.

We have observed the same mechanism with the external frequency $\omega=e / 4$ (with a relative distance less than $10 \%$ of the golden mean). This number has very different Diophantine properties from those of the golden mean; in particular, it does not have a periodic continuous fraction expansion. (The continued fraction expansion of $e / 4$ is known explicitly and has unbounded entries.)

4. Example 2: The rotating standard map. Our second example is the rotating standard map. This is a quasi-periodic perturbation of the standard map [Chi79, Gre79], that is, a symplectic (area preserving) map of the annulus $\mathbb{T} \times \mathbb{R}$.

We will study the continuation of hyperbolic invariant tori, whose theory has been developed. Moreover, we will also study continuation of elliptic tori and the bifurcation of elliptic tori. We think that it would be quite desirable to develop rigorous a posteriori estimates for these cases. We will also describe another version of the bundle merging bifurcation, which has some quantitative differences from the one studied in section 3.2.

4.1. Preliminaries and notation. The rotating standard map is given by

$$
\begin{aligned}
& \bar{x}=x+y-s(x)(K+\varepsilon c(\theta)) \quad(\bmod 1), \\
& \bar{y}=\quad y-s(x)(K+\varepsilon c(\theta)) \text {, } \\
& \bar{\theta}=\theta+\omega \quad(\bmod 1),
\end{aligned}
$$


where

$$
s(x)=\frac{1}{2 \pi} \sin (2 \pi x)
$$

is a 1-periodic odd function,

$$
c(\theta)=\cos (2 \pi \theta)
$$

is a 1-periodic even function, and $K$ and $\varepsilon$ are positive parameters. We will refer to $y, x$ as the action-angle variables and $\omega$ as the external frequency.

So, the extended phase space is $\mathbb{T} \times \mathbb{R} \times \mathbb{T}$. We have taken $\omega=\tau-1$, where $\tau$ is the largest root of the polynomial $p(t)=t^{3}-t^{2}-t-1$. That is,

$$
\omega=\sqrt[3]{\frac{19}{27}+\sqrt{\frac{11}{27}}}+\sqrt[3]{\frac{19}{27}-\sqrt{\frac{11}{27}}}-\frac{2}{3} .
$$

This choice of an irrational number is motivated by this number's usefulness in the study of 2D KAM tori. See [Har98]. For our purposes, it is useful to check the properties when the frequency is different from the golden mean. Note that the number above does not have a periodic continued fraction expansion.

The rotating standard map is reversible (as is the standard map), since it is the composition $F=I_{1} \circ I_{0}$ of two involutions $I_{0}$, given by

$$
\begin{aligned}
& \bar{x}=-x \quad(\bmod 1), \\
& \bar{y}=y-s(x)(K+\varepsilon c(\theta)), \\
& \bar{\theta}=-\theta \quad(\bmod 1),
\end{aligned}
$$

and $I_{1}$, given by

$$
\begin{aligned}
& \bar{x}=-x+y \quad(\bmod 1), \\
& \bar{y}=y, \\
& \bar{\theta}=-\theta+\omega \quad(\bmod 1) .
\end{aligned}
$$

As far as we know, the rotating standard map was introduced in [ACS91, ACS92], where some properties of the map and existence of KAM tori were investigated. This example was also studied in [Tom96], which used a higher dimensional extension of Greene's criterion [Gre79] to study the breakdown of a 2D invariant KAM torus. The thesis [Har98] contains a study of the breakdown of the 2D KAM tori using variational principles.

The standard map, corresponding to $\varepsilon=0$, is an area preserving map that has periodic orbits of all rotation numbers. We say that the rotation number of an $n$-periodic orbit through $\left(x_{0}, y_{0}\right)$ is $p / n$ if $x_{n}=x_{0}+p, y_{n}=y_{0}$, where $\left(x_{n}, y_{n}\right)=F^{n}\left(x_{0}, y_{0}\right)$ and $F$ is the lift of the standard map to $\mathbb{R} \times \mathbb{R}$ (that is, we think of $x$ as a real variable instead of as an angle).

Analogously, we can define the rotation number of a $n$-periodic curve $K_{0}(\theta)=\left(x_{0}(\theta), y_{0}(\theta)\right)$ of the rotating standard map as the rational number $p / n$ such that

$$
x_{n}(\theta)=x_{0}(\theta+n \omega)+p, y_{n}(\theta)=y_{0}(\theta+n \omega),
$$


where $K_{n}(\theta)=F(\theta, n)\left(K_{0}(\theta)\right)=\left(x_{n}(\theta), y_{n}(\theta)\right)$ is the $n$-iterate of the torus $K_{0}$.

Notice that the tori $\{x=0, y=0\}$ and $\left\{x=\frac{1}{2}, y=0\right\}$ are invariant under the rotating standard map. Their rotation numbers are both $0 / 1$. If $\varepsilon=0$, the torus $\{x=0, y=0\}$ corresponds to an elliptic fixed point if $0<K<4$ and to a hyperbolic fixed point (with reflection) if $K>4$. It is parabolic if $K=4$. The torus $\left\{x=\frac{1}{2}, y=0\right\}$ for $\varepsilon=0$ corresponds to a hyperbolic fixed point if $K>0$.

Notice also that the torus $\left\{x=0, y=\frac{1}{2}\right\}$ is a 2-periodic curve under the rotating standard map, whose rotation number is $1 / 2$. If $\varepsilon=0$, it corresponds to an elliptic 2-periodic orbit if $K<\sqrt{2}$ and to a hyperbolic 2-periodic orbit if $K>\sqrt{2}$.

In our numerical experiments that are described below, we have fixed $K=0.2$ and changed the forcing constant $\varepsilon$.

4.2. A 2-periodic normally hyperbolic torus. The simplest nontrivial periodic torus for the rotating standard map with $K>0$ is generated from $\varepsilon=0$ by the hyperbolic periodic orbit of rotation number $1 / 2$. We also compute this 2 -periodic saddle curve using the reducibility method, and the results are displayed in Table 2 and Figure 7.

Once we have computed a 2-periodic saddle curve, we compute its stable and unstable invariant manifolds. We have expanded them to order 30 and then we have globalized them. Our programs produce 2D grids of those objects, which we display in Figure 8, for $K=0.2$, $\varepsilon=0.5$. We have also made some slices of those objects, producing the familiar homoclinic web. This is displayed in Figure 9. Keep in mind that these are slices of 2D objects in a 3D space. Notice that the objects we produce are symmetric. This is due to the reversibility of the rotating standard map.

4.3. Continuation of reducibility. In this section we will analyze the applicability of the reducibility method to normally elliptic tori. Later, we will discuss some bifurcations that can arise when the reducibility fails.

As a first example, we will consider the torus $\{x=0, y=0\}$ of the rotating standard map, which is invariant for all the values $K, \varepsilon$. The normal behavior is given by the cocycle

$$
M(\theta)=\left(\begin{array}{rr}
1-(K+\varepsilon \cos (2 \pi \theta)) & 1 \\
-(K+\varepsilon \cos (2 \pi \theta)) & 1
\end{array}\right)
$$

with rotation $\omega$. So then, the reducibility method provides approximations of the reduced matrices and the corresponding Floquet transformations.

This example has the advantage that the torus is fixed for all the values of the parameters, and so then the only thing we have to do is study the cocycle (30). We will refer to it as the standard cocycle. This example serves as a test and gives some insight about the general case, and the effects that happen at breakdown of reducibility are easier to study. A continuation of elliptic tori that are not fixed by the symmetry will be undertaken later in this section.

Remark 4.1. The standard cocycle (30) is equivalent to the almost Mathieu cocycle

$$
A(\theta)=\left(\begin{array}{cc}
a-b \cos (2 \pi \theta) & -1 \\
1 & 0
\end{array}\right)
$$




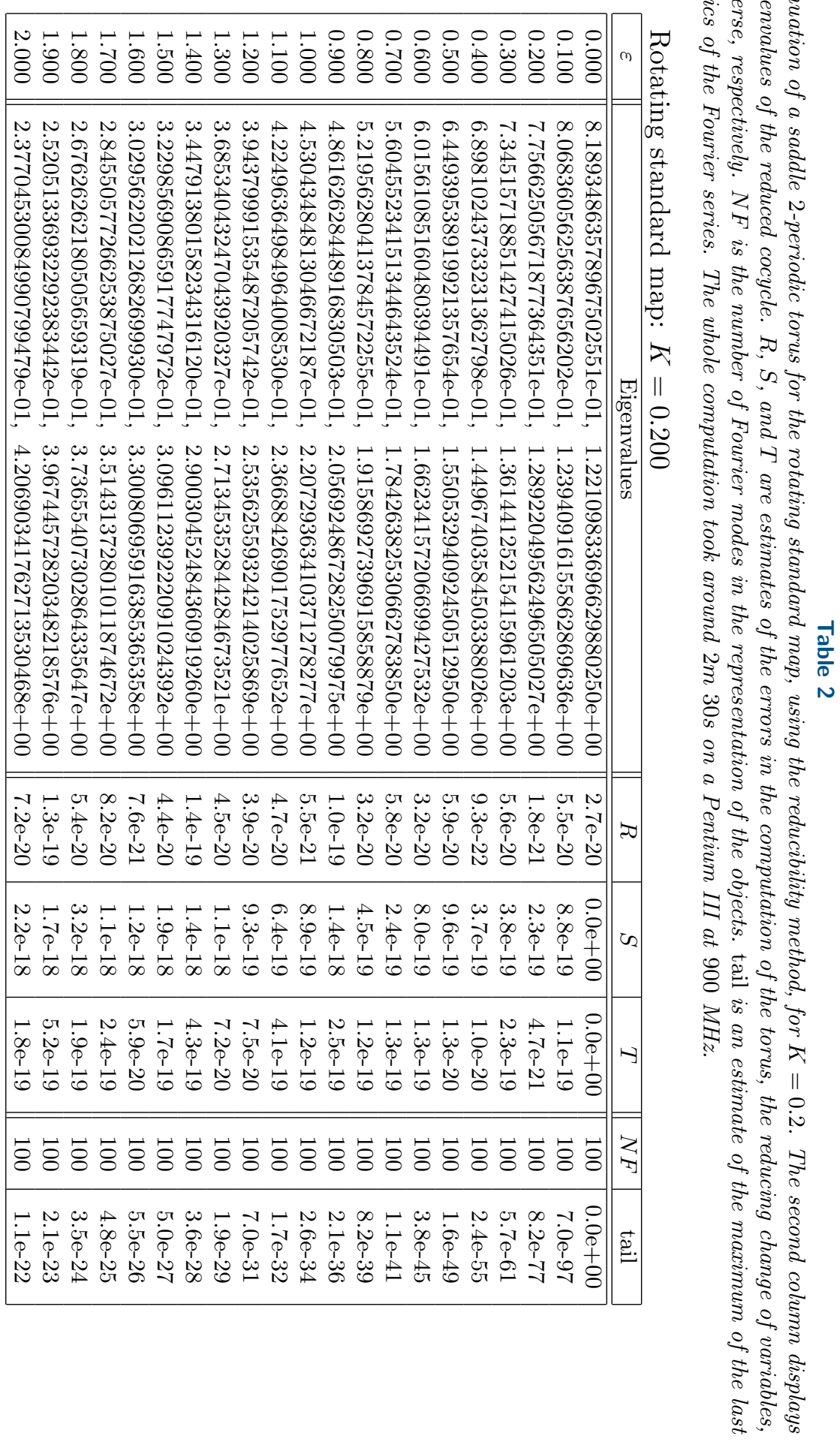

จำำ

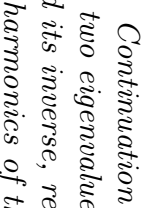

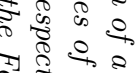

के के

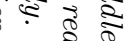

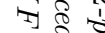

क. 8 है

क क

हूँ సू हु

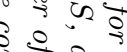

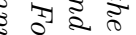

3 范

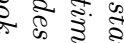

胥.

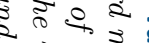

궁

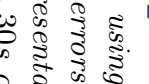

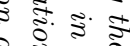

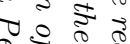

.

윤

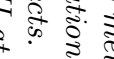

\&

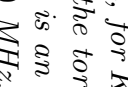

है है

章

온

ปี่

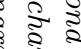

के

远

से है

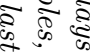



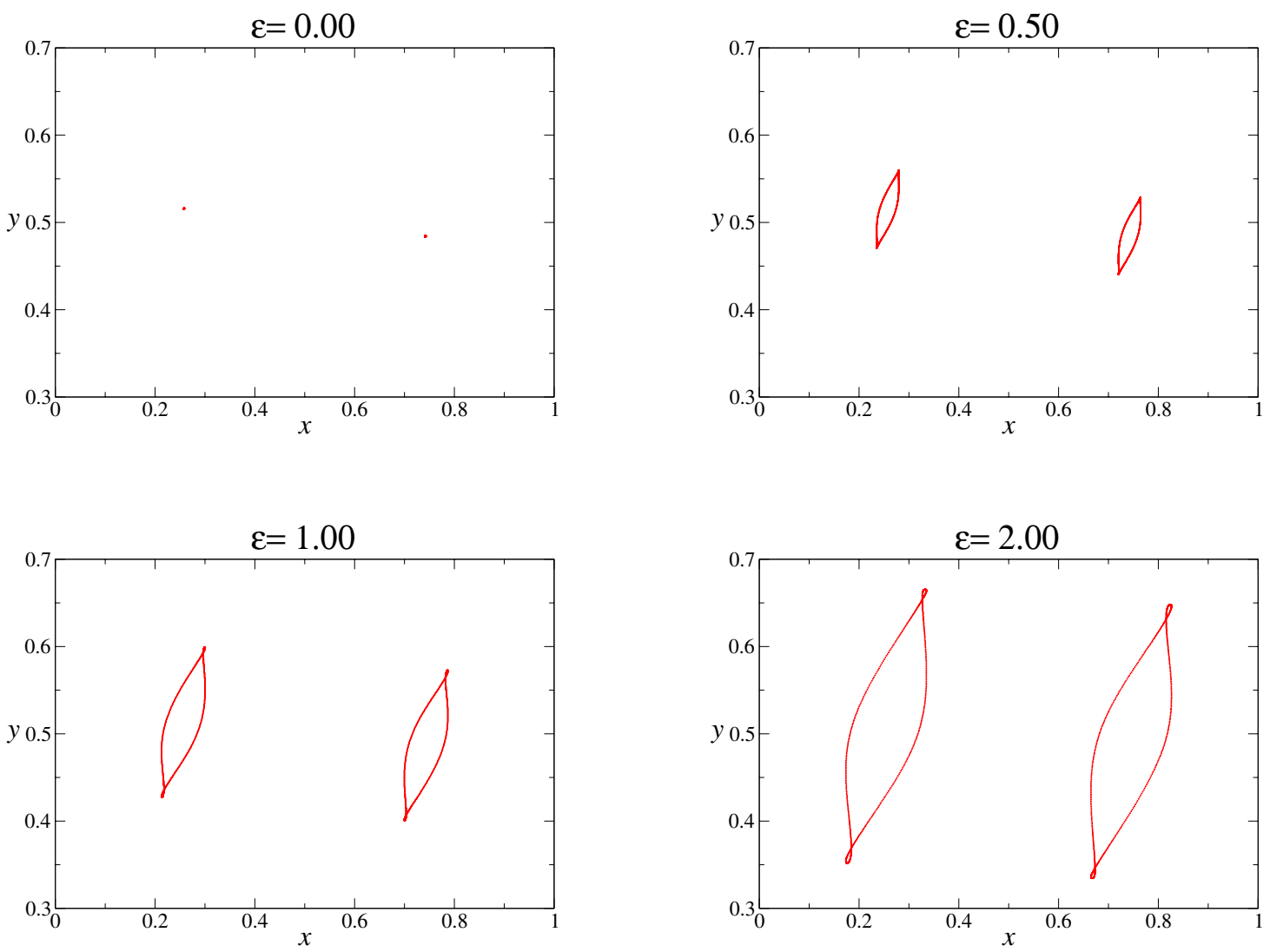

Figure 7. $x y$ projection of a 2-periodic saddle curve for the rotating standard map $(K=0.20)$.

with rotation $\omega$, where $a=2-K, b=\varepsilon$. In fact, $M(\theta) P=P A(\theta)$, where $P$ is the constant antisymplectic matrix

$$
\left(\begin{array}{cc}
1 & 0 \\
1 & -1
\end{array}\right)
$$

The almost Mathieu cocycle has called the attention of many authors, among them [CEY90, BS82, Las94, AS82, AS83, DS83a, Eli01, Jit99, AK03, Pui04].

We have analyzed numerically the standard cocycle for $K=0.2$, moving the parameter $\varepsilon$. We have applied the reducibility method to reduce the linear cocycle. The problem of reducibility has been considered in [Eli88, Pös89, JS92, Pui05]. There is a Cantor family of parameters for which the cocycle is reducible and elliptic. There is also an open set of parameters for which the cocycle is normally hyperbolic (and reducible). The results are displayed in Table 3.

4.3.1. Detection of resonances in elliptic tori. We have observed that the program implementing the reducibility method cannot continue beyond the critical value

$$
\varepsilon_{\text {crit }} \simeq 0.0963488851723619376131 .
$$




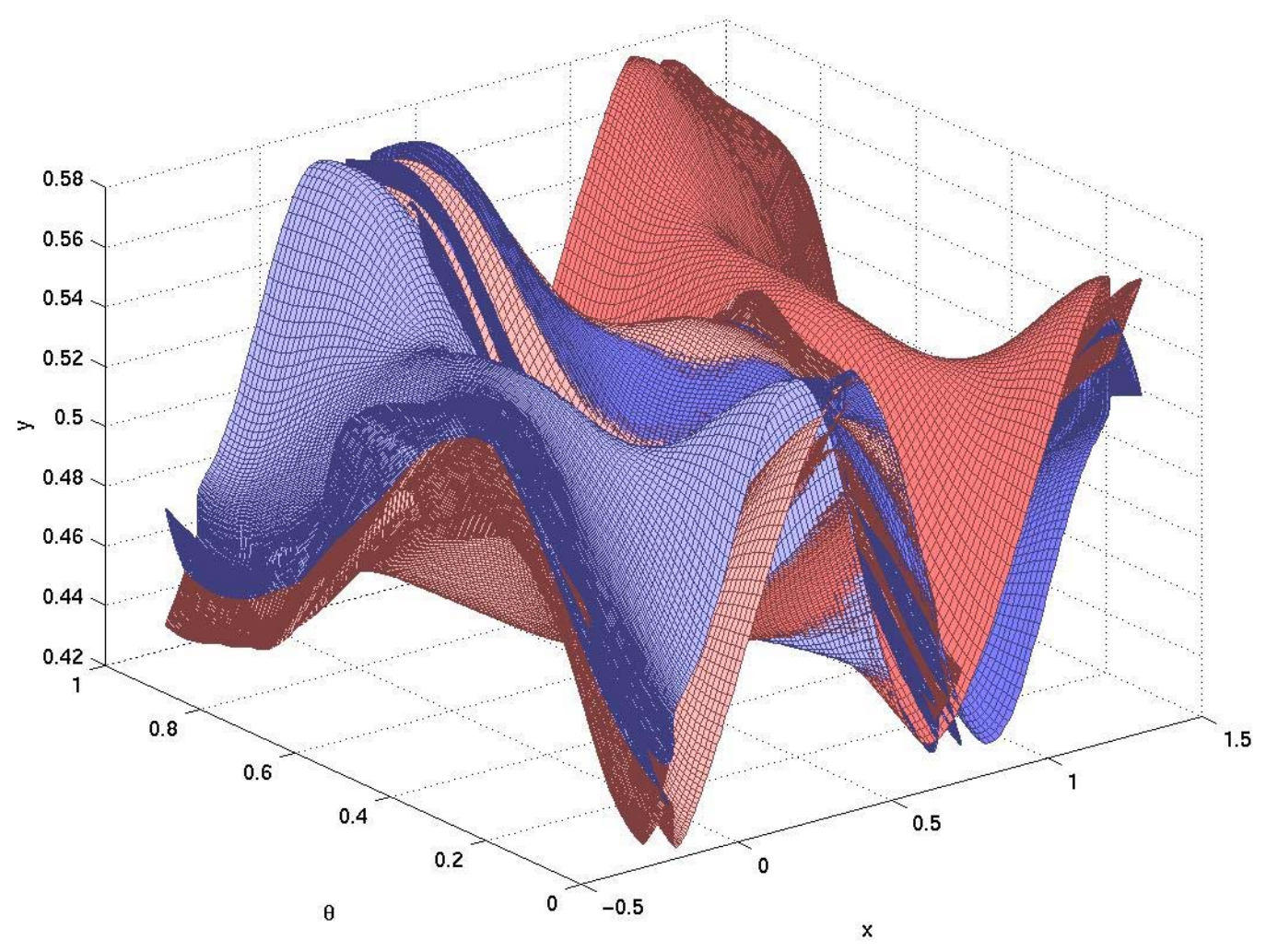

Figure 8. $3 D$ view of the stable (blue) and unstable (red) manifolds of a hyperbolic 2-periodic circle for the rotating standard map. The parameters are $K=0.2$ and $\varepsilon=0.5$, and the frequency of the forcing is $\omega=\tau-1$ (see text for details).

The reason is that for such a value the rotation number $\gamma$ of the cocycle (the argument of the eigenvalue) and the external frequency $\omega$, which are

$$
\gamma \simeq-0.504895542331356775420, \omega \simeq 0.83928675521416126683
$$

are very close to $-1: 1$ resonance:

$$
\left|\frac{\gamma}{2 \pi}-\frac{k_{1}+k_{2} \omega}{2}\right| \simeq 1.08582110^{-9}
$$

for $k_{1}=-1, k_{2}=1$. It is not hard to show that, when there is a resonance, if there is a nonlinear term, this provides an obstruction to reducibility. So, it is quite possible that there is a dense set of parameter values for which reducibility - in the mathematical sense - fails. On the other hand, we note that, when the order of the resonance is high, the interval shows only on high order perturbation theory. Hence, it seems that the measure of the gaps except those of resonances whose numerator $k_{2}$ is small will be visible.

4.3.2. Analysis of the cocycle. We have computed the dominant Lyapunov multiplier and the rotation number of the standard cocycle [BS98]. To do so, we have considered orbits 

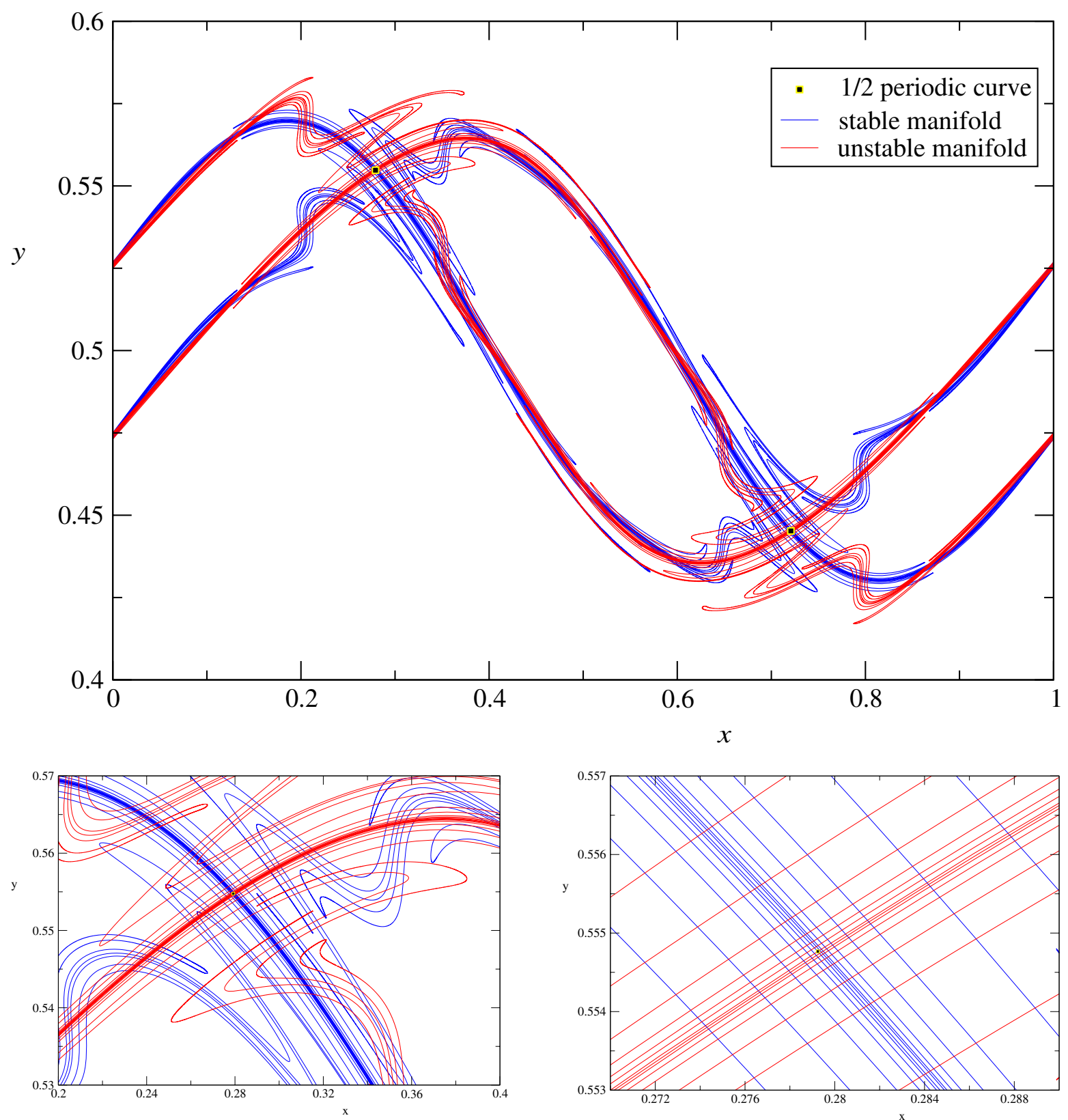

Figure 9. Heteroclinic web associated with the hyperbolic 2-periodic circle. The section of the stable and unstable manifolds is with $\theta=0$. Both of the lower figures are magnifications of the first one.

of length $10^{8}$ of the cocycle (applied to a random vector). The results are displayed in the two last columns of Table 3 (and agree with the computed eigenvalues in about 7 digits) and in Figure 10. We note that just after the critical value $\varepsilon_{\text {crit }}$, the maximal Lyapunov multiplier is appreciably bigger than 1 , and the rotation number is phase locked in the angle $\gamma$, so the torus bifurcates into a normally hyperbolic torus. 


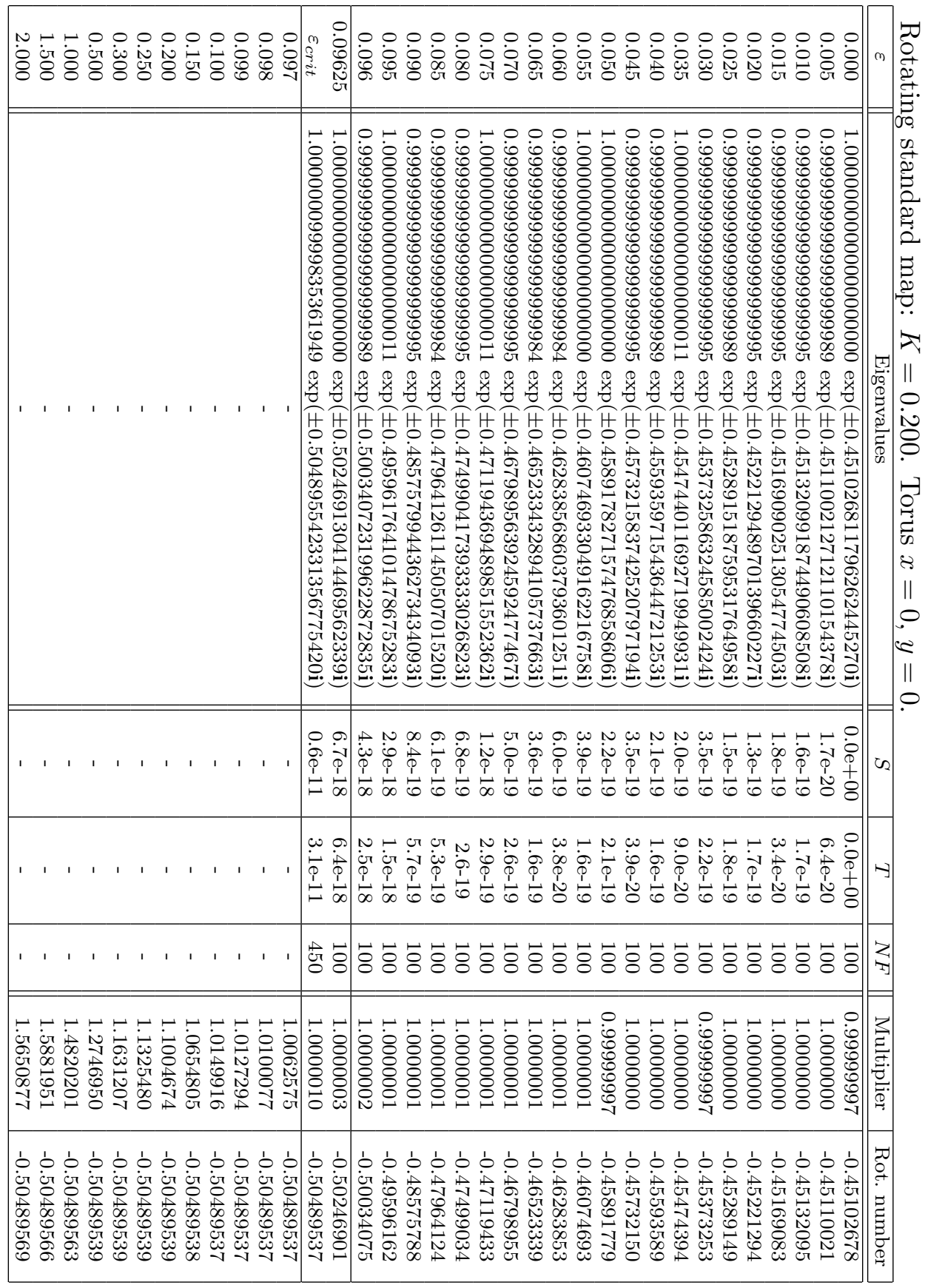

उ $\mathrm{\Omega}$ है है

ส․ำ

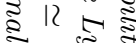

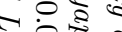

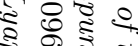

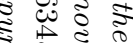

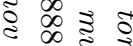

उ

हैं

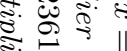

จ.

is 9 o 2 ic

\&

$\mathbb{8}^{8}, \overrightarrow{0}$

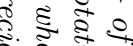

골

2.

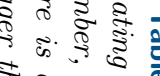

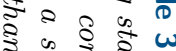

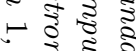

ह वह है है

है $\overrightarrow{5}$

₹

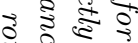

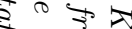

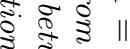

ฐู

क.

ธ. ฐँ के

จ.

दू हू ह

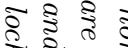

है

$\infty$ के बै

₹

争.

है

का के के

क क है

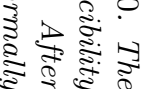


Remark 4.2. From [Her83], the maximal Lyapunov multiplier of the almost Mathieu cocycle (31) (and so of the standard cocycle (30)) is bounded from below by $\frac{\varepsilon}{2}$. In fact, if the almost Mathieu cocycle is not uniformly hyperbolic, then the maximal Lyapunov multiplier is $l(\varepsilon)=\max \left(1, \frac{\varepsilon}{2}\right)$ [BJ02]. See Figure 10, where we also display the lower bound $l(\varepsilon)$.

We distinguish several regions in Figure 10. Notice that after the critical value, the dominant Lyapunov multiplier is bigger than 1 , so the torus is not going to be elliptic anymore. In such a case, the spectrum of the transfer operator associated to the cocycle is going to be either two circles or a fat annulus (notice that the radii are inverses of each other).

When the rotation number is phase locked then the spectrum is two circles and the cocycle has stable and unstable invariant subbundles, so the torus is normally hyperbolic. (And the type of resonance has to do with the index of the stable and unstable subbundles.)

In the regions where the rotation number is not phase locked (and the Lyapunov multiplier is bigger than 1), the torus is nonuniformly hyperbolic. Notice that a nonuniformly hyperbolic torus is destroyed by generic perturbations [Mn78], but in the present example the torus survives because the perturbation vanishes on the torus.

We can confirm our expectations using the trick of projectivization described in section 2.2.1. The examples we display in Figure 11 correspond to the regions detected in Figure 10.

The following is an analysis of both Figure 10 and Figure 11.

- From $\varepsilon=0$ to $\varepsilon \simeq 0.096$ the torus is "almost" elliptic (there are small gaps in which the torus loses its ellipticity). See Figure 11 for $\varepsilon=0.050$ and $\varepsilon=0.095$, where the dynamics of the projective cocycle is "almost" ergodic and a strong resonance is born.

- From $\varepsilon \simeq 0.096$ to $\varepsilon \simeq 2.345$ the torus is normally hyperbolic. This happens for a whole open interval that in this case is a big gap corresponding to a $-1: 1$ resonance. Moreover, the indices of the stable and unstable subbundles are $\frac{1}{2}=\frac{k_{2}}{2}$, where $k_{2}=1$ in (32), and as a result those subbundles are nonorientable. See Figure 11 for $\varepsilon=0.100$, $\varepsilon=0.500$, and $\varepsilon=1.000$.

- From $\varepsilon \simeq 2.345$ to $\varepsilon \simeq 2.697$ there are two different behaviors that coexist: the torus is normally hyperbolic in the small bumps in Figure 10, which are open intervals (moreover, the unstable and stable bundles probably have a large index), and the torus is nonuniformly hyperbolic in the complementary intervals. See Figure 11 for $\varepsilon=0.250$, where the attractor and repellor of the projective cocycle collapse.

- From $\varepsilon \simeq 2.697$ to $\varepsilon \simeq 4.332$ the torus is again normally hyperbolic for a large open interval. The resonance is $-2: 2$, and the invariant subbundles have index $1=\frac{2}{2}$, so they are orientable. See Figure 11 for $\varepsilon=4.000$.

- From $\varepsilon \simeq 4.332$ to $\varepsilon=5.000$, where we stop our analysis, we are again in a normally/ nonuniformly hyperbolic region. See Figure 11 for $\varepsilon=5.000$.

To finish the analysis of the standard cocycle we come back to our starting point in this section: the detection of $\mathrm{a}-1: 1$ resonance between the internal and normal frequencies that prevent the continuation of the reducibility (notice that we enter in a region that, in a sense, is "more reducible" than in the first elliptic region). We emphasize that we have detected this phenomenon numerically because the resonance is very strong, producing a big gap. But it occurs also in very small gaps. Numerical and rigorous results on these kinds of gaps and the corresponding bifurcations appear in $\left[\mathrm{BHJ}^{+} 03\right]$ for Hamiltonian systems. 

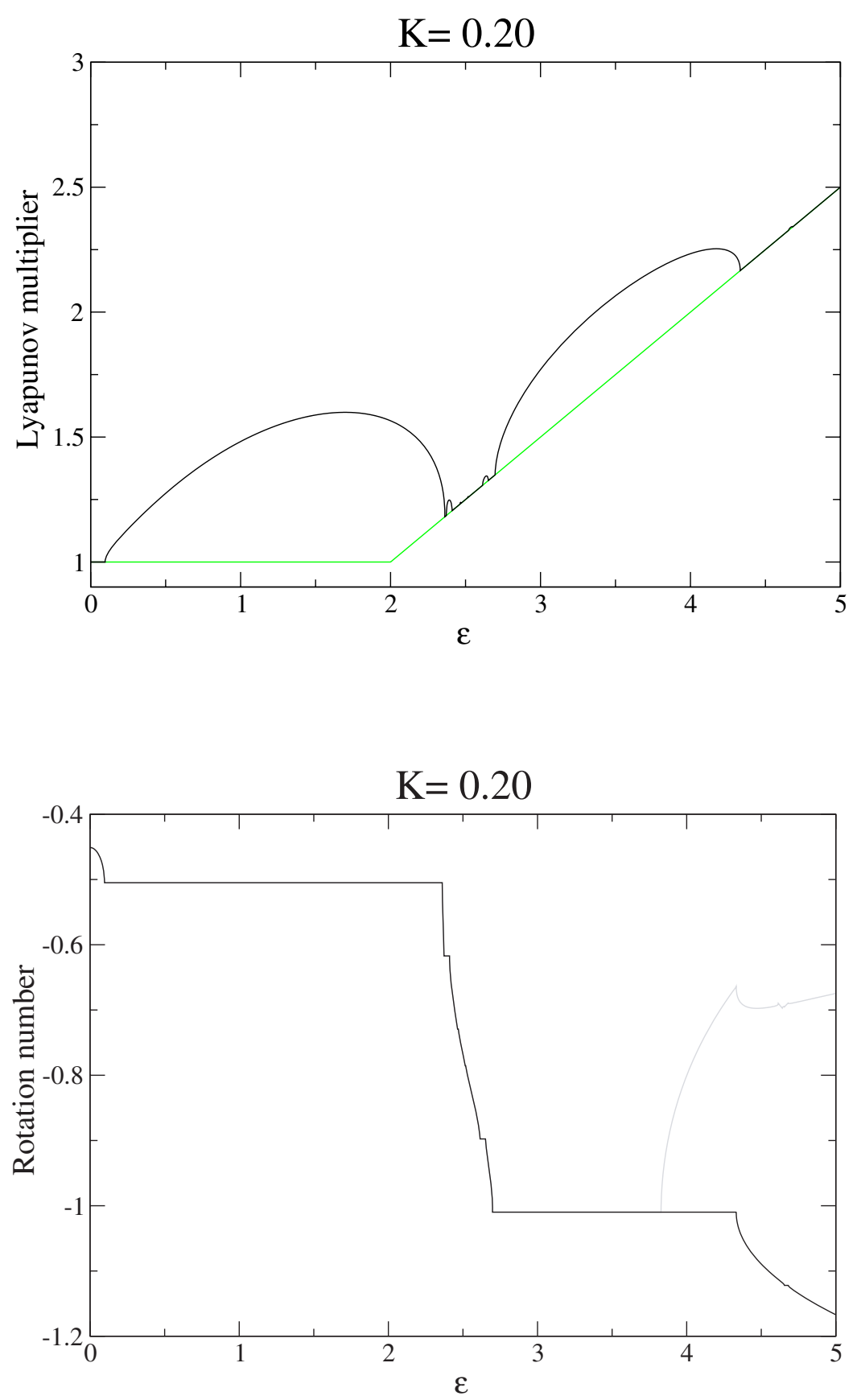

Figure 10. Lyapunov multiplier and rotation number as functions of $\varepsilon$ of the standard cocycle (30) with $\omega=\tau-1, K=0.2$. The green function is a lower bound of the Lyapunov multiplier: $l(\varepsilon)=\max \left(1, \frac{\varepsilon}{2}\right)$. The rotation number has been computed with the Sturmian definition (19). We also display the average rotation number (grey color) that produces incorrect estimates beyond $\varepsilon \simeq 3.829 \simeq 4-K$. 

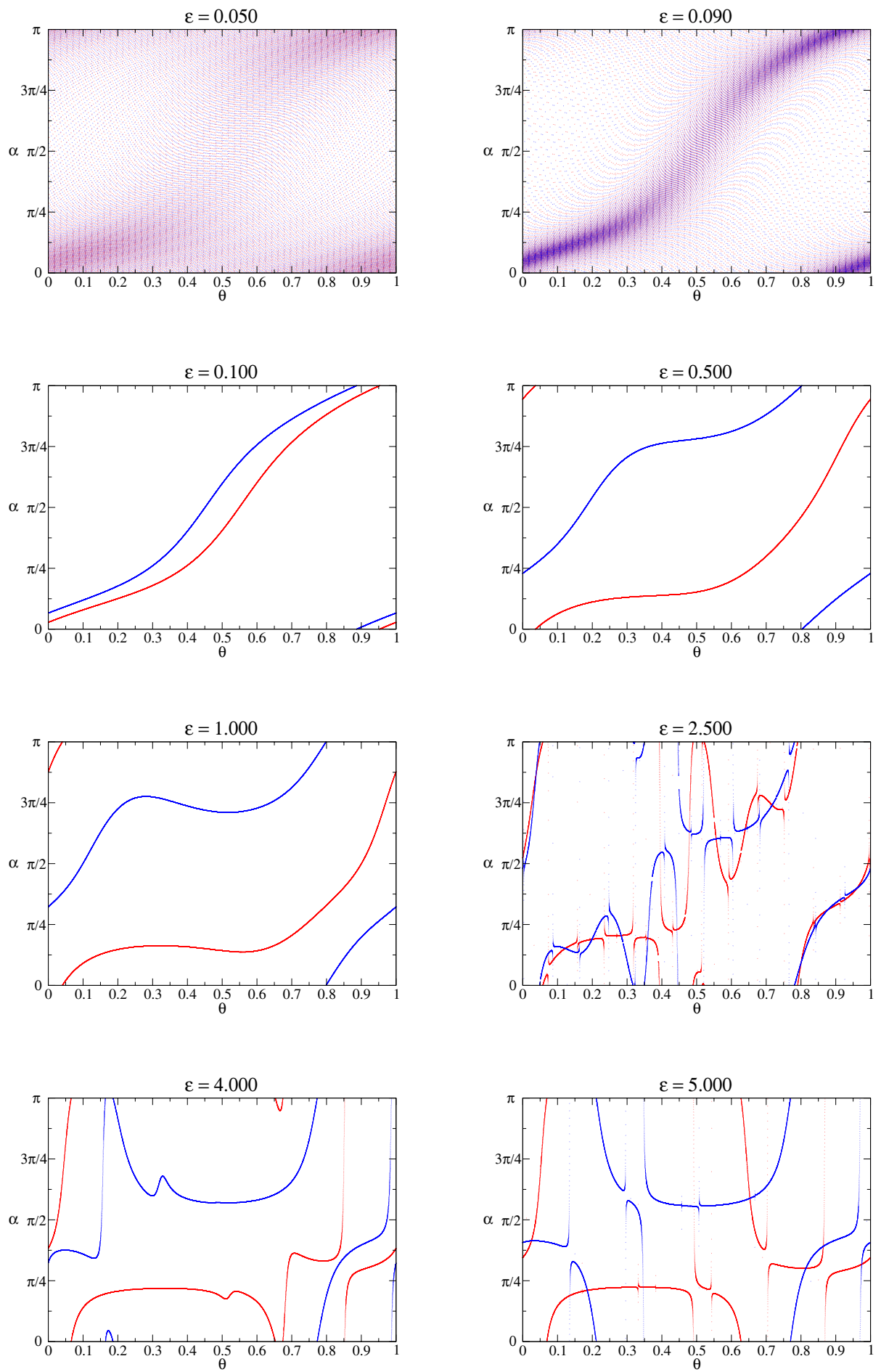

Figure 11. Attractor (red) and repellor (blue) of the projective standard cocycle with $K=0.2$. This is associated to the torus $\{x=0, y=0\}$ that is invariant for the rotating standard map. 
Table 4

Continuation of reducibility and Floquet transformation of the torus $\{x=0, y=0\}$ of the rotating standard map for $K=0.2$. We improve reducibility using rotating transformations, so we can cross the resonance $-1: 1$ that appears in $\varepsilon=\varepsilon_{\text {crit }} \simeq 0.0963488851723619376131$.

Rotating standard map: $K=0.200$. Torus $\{x=0, y=0\}$.

\begin{tabular}{|c|c|c|c|c|}
\hline$\varepsilon$ & Eigenvalues & $S$ & $T$ & $N F$ \\
\hline 0.000 & $1.00000000000000000000 \exp ( \pm 0.45102681179626244527 \mathbf{i})$ & $0.0 \mathrm{e}+00$ & $0.0 \mathrm{e}+00$ & 100 \\
\hline 0.010 & $0.99999999999999999995 \exp ( \pm 0.45132099187449060824 \mathbf{i})$ & $7.7 \mathrm{e}-20$ & $6.9 \mathrm{e}-20$ & 100 \\
\hline 0.020 & $0.99999999999999999984 \exp ( \pm 0.45221294897013966028 \mathbf{i})$ & $1.4 \mathrm{e}-19$ & $1.0 \mathrm{e}-20$ & 100 \\
\hline 0.030 & $1.00000000000000000011 \exp ( \pm 0.45373258632458500232 \mathbf{i})$ & $1.6 \mathrm{e}-19$ & $9.3 \mathrm{e}-20$ & 100 \\
\hline 0.040 & $1.00000000000000000000 \exp ( \pm 0.45593597154364472120 \mathbf{i})$ & $2.1 \mathrm{e}-19$ & $1.5 \mathrm{e}-19$ & 100 \\
\hline 0.050 & $1.00000000000000000000 \exp ( \pm 3.09561493159350098947 \mathbf{i})$ & $5.1 \mathrm{e}-10$ & $3.2 \mathrm{e}-19$ & 100 \\
\hline 0.060 & $1.00000000000000000000 \exp ( \pm 3.09953567303981773143 \mathbf{i})$ & $3.4 \mathrm{e}-19$ & $2.3 \mathrm{e}-19$ & 100 \\
\hline 0.070 & $0.99999999999999999995 \exp ( \pm 3.10468666836061660871 \mathbf{i})$ & $5.2 \mathrm{e}-19$ & $2.5 \mathrm{e}-19$ & 100 \\
\hline 0.080 & $1.00000000000000000000 \exp ( \pm 3.11168752182935715836 \mathbf{i})$ & $2.6 \mathrm{e}-19$ & $3.0 \mathrm{e}-19$ & 100 \\
\hline 0.090 & $1.00000000000000000000 \exp ( \pm 3.12245509887229756346 \mathbf{i})$ & $3.8 \mathrm{e}-19$ & $5.1 \mathrm{e}-19$ & 100 \\
\hline 0.100 & $-0.985229910849008448355, \quad-1.01499151516650933645$ & $3.0 \mathrm{e}-19$ & $2.7 \mathrm{e}-19$ & 100 \\
\hline 0.200 & -0.908704733983905314765 & $3.3 \mathrm{e}-19$ & $3.6 \mathrm{e}-19$ & 100 \\
\hline 0.300 & -0.859755912104609600457 & $4.6 \mathrm{e}-19$ & $3.2 \mathrm{e}-19$ & 100 \\
\hline 0.400 & -0.819034502317305008415 & $5.7 \mathrm{e}-19$ & $2.2 \mathrm{e}-19$ & 100 \\
\hline 0.500 & -0.784499412393390937147 & $3.7 \mathrm{e}-19$ & $2.5 \mathrm{e}-19$ & 100 \\
\hline 0.600 & -0.755089133897168369623 & $5.4 \mathrm{e}-19$ & $4.7 \mathrm{e}-19$ & 100 \\
\hline 0.700 & -0.729998333578531503093 & $4.6 \mathrm{e}-19$ & $3.4 \mathrm{e}-19$ & 100 \\
\hline 0.800 & -0.708575796553274791698 & $7.5 \mathrm{e}-19$ & $3.1 \mathrm{e}-19$ & 100 \\
\hline 0.900 & -0.690298554554738629245 & $4.8 \mathrm{e}-19$ & $3.7 \mathrm{e}-19$ & 100 \\
\hline 1.000 & -0.674754590255442611413 & $8.0 \mathrm{e}-19$ & $2.9 \mathrm{e}-19$ & 100 \\
\hline 1.500 & -0.629645514281034828278 & $1.1 \mathrm{e}-16$ & $7.8 \mathrm{e}-17$ & 100 \\
\hline 2.000 & -0.638941822211735963705 & $1.8 \mathrm{e}-09$ & $1.1 \mathrm{e}-09$ & 100 \\
\hline 2.300 & -0.721367120200611090905 & $5.4 \mathrm{e}-08$ & $2.4 \mathrm{e}-08$ & 300 \\
\hline
\end{tabular}

4.3.3. Bifurcations at resonance in elliptic tori. The reducibility method stops at the resonance because the Floquet transformation is not well adapted to the topology of the stable and unstable subbundles produced after the critical value $\varepsilon_{\text {crit }}$. Nevertheless, we will see that it is possible to continue the torus and its reducibility if we take into account that invariant bundles can appear, and therefore the torus can become normally hyperbolic.

We have to take into account the somewhat unexpected phenomenon that the invariant bundles generated by the resonance have an index which is related to the resonance. In particular, they may be nonorientable.

The key idea is to use the rotating transformations of section 2.2.6. To apply such a transformation it is not necessary to wait until the resonance is more evident (say, to values very close to $\left.\varepsilon_{\text {crit }}\right)$. In fact, our experiments suggest that it is better numerically to do this transformation before the transition happens. In Table 4 we display the results when doing the transformation for $\varepsilon=0.050$, quite far from $\varepsilon_{\text {crit }} \simeq 0.0963489$.

We see that the method continues the reducibility of the normally hyperbolic torus, whose cocycle has a spectrum composed by two circles, until there is a new transition near $\varepsilon \simeq 2.345$. Again, reducibility is lost and the method stops. Notice, however, that in this case, the torus is nonuniformly normally hyperbolic. The spectrum grows and it is a full annulus enclosed by two circles, and the Oseledec bundles are not continuous. 


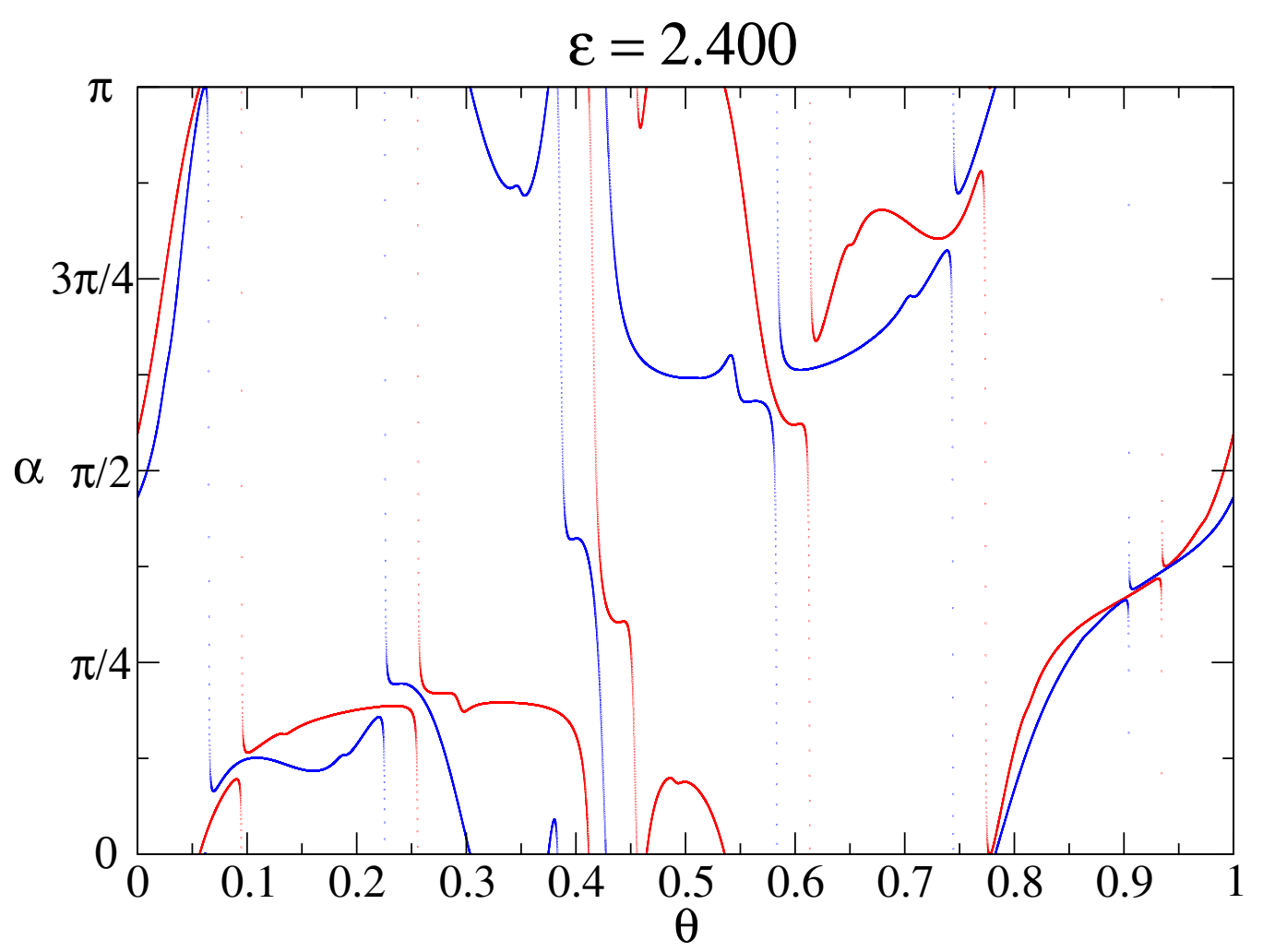

Figure 12. Attractor (red) and repellor (blue) of the projective cocycle of the invariant torus $\{x=0, y=0\}$ of the rotating standard map with $K=0.2$ and $\varepsilon=2.400$.

Notice, moreover, that reducibility holds in the small bumps between 2.345 and 2.697, and we can do a direct analysis by using projectivization. We can compute the invariant bundles using the power method [HdlL05a] or by discretizing the transfer operator [Jor99].

4.3.4. Topological evidence of bundle collapse (II). The invariance of the index of the bundles under homotopies has some consequences. By observing at some ends, we may conclude that something has happened in the middle.

For example, we observe that the torus is normally hyperbolic for $\varepsilon=2.400$, with resonance $4:-5$, so its invariant manifolds have index $\frac{-5}{2}$ and are nonorientable (see Figure 12). Since for $\varepsilon=2.340$ the torus is normally hyperbolic and the invariant manifolds have index $\frac{1}{2}$, the torus cannot be normally hyperbolic for all the values between $\varepsilon=2.340$ and $\varepsilon=2.400$. Since the Lyapunov multiplier is bounded away from 1 (see Remark 4.2), the only possibility is that the stable and unstable bundles merge at some values of the parameter $\varepsilon$ between 2.340 and 2.400 .

Notice also that the torus is again normally hyperbolic for $\varepsilon=0.4$, and the index of the invariant bundle in this case is 1 , so that there are further transitions. 
4.4. Nonorientable whiskers generated at resonances. We note that the rotating transformations introduced in section 2.2.6 lead to the existence of nontrivial invariant bundles in the linearization of the invariant circle $\{x=0, y=0\}$ in the standard map. In particular, we have found cases in which those bundles are nonorientable.

As remarked before, the methods of this paper can compute invariant manifolds modeled in these invariant bundles for the linearization.

Figure 13 shows the stable and unstable manifolds of the invariant torus $\{x=0, y=0\}$ for the rotating standard map, with $K=0.2, \varepsilon=0.5$.

4.5. Continuation of an invariant torus until breakdown. In this section we consider the continuation of the elliptic 3-periodic orbit of the standard map with $K=0.2$.

Using the reducibility method, we detect a strong resonance for the value

$$
\varepsilon_{\text {crit }} \simeq 0.06078370936559205612
$$

for which the rotation number is

$$
\gamma \simeq-0.112219351279490344844
$$

In fact,

$$
\left|\frac{\gamma}{2 \pi}-\frac{k_{1}+k_{2} 3 \omega}{2}\right| \simeq 1.17578710^{-9}
$$

for $k_{1}=5, k_{2}=-2$, so the resonance is of the type $5:-2$. This suggests the transition to a normally hyperbolic torus whose subbundles have index $\frac{-2}{2}=-1$, so they are orientable. We can cross the resonance and compute the unstable and stable subbundles of the normally hyperbolic torus using the rotating transformation $R_{2}$ (see section 2.2.6).

The results of the full continuation are displayed in Figure 14 (Lyapunov multipliers and rotation number), Figure 15 ( $\theta x$ projection of the 3-periodic torus), and Figure 16 (dynamics of the projective cocycle).

- From $\varepsilon=0$ to $\varepsilon \simeq 0.061$ the torus is elliptic (outside of small resonance intervals that are not detected numerically with the continuation step 0.001), and the dynamics of the projective cocycle is ergodic. See Figure 16 for $\varepsilon=0.050$.

- From $\varepsilon \simeq 0.061$ to $\varepsilon \simeq 0.117$ the torus is normally hyperbolic and the invariant subbundles have index -1 . See Figure 16 for $\varepsilon=0.100$. Moreover, the eigenvalues are negative. For instance, for $\varepsilon=0.100$, the eigenvalues are

$$
-0.990944333,-1.0091384215 \text {. }
$$

- From $\varepsilon \simeq 0.117$ to $\varepsilon \simeq 0.173$ the torus is again elliptic. See Figure 16 for $\varepsilon=0.150$.

- From $\varepsilon \simeq 0.173$ to $\varepsilon \simeq 0.350$ the torus is normally hyperbolic and the invariant subbundles again have index -1 . See Figure 16 for $\varepsilon=0.200,0.250,0.300,0.350$. The eigenvalues are again negative. For instance, for $\varepsilon=0.200$, the eigenvalues are

$$
-0.963855575,-1.0374998344 \text {. }
$$




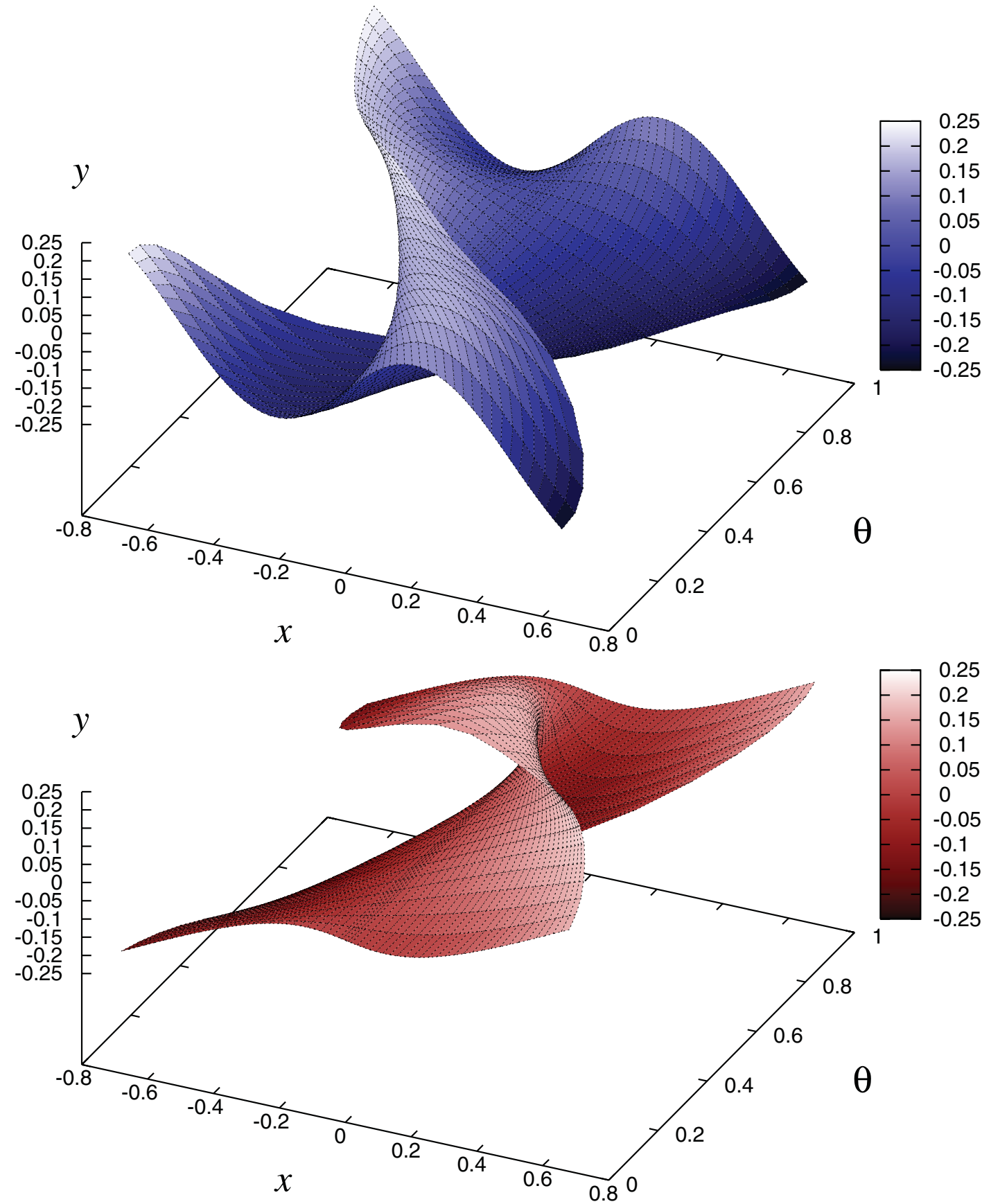

Figure 13. $3 D$ views of the stable (blue) and unstable (red) manifolds of the curve $\{x=0, y=0\}$ for the rotating standard map with $\kappa=0.2$ and $\varepsilon=0.5$. Both manifolds are nonorientable.

We observe also a similar phenomena to that in section 3.2. After the second strong resonance (that we can continue with reducibility because we adapted the coordinates to the topology already in the first resonance, and both of them are $5:-2$ ), the torus is a saddle-type 

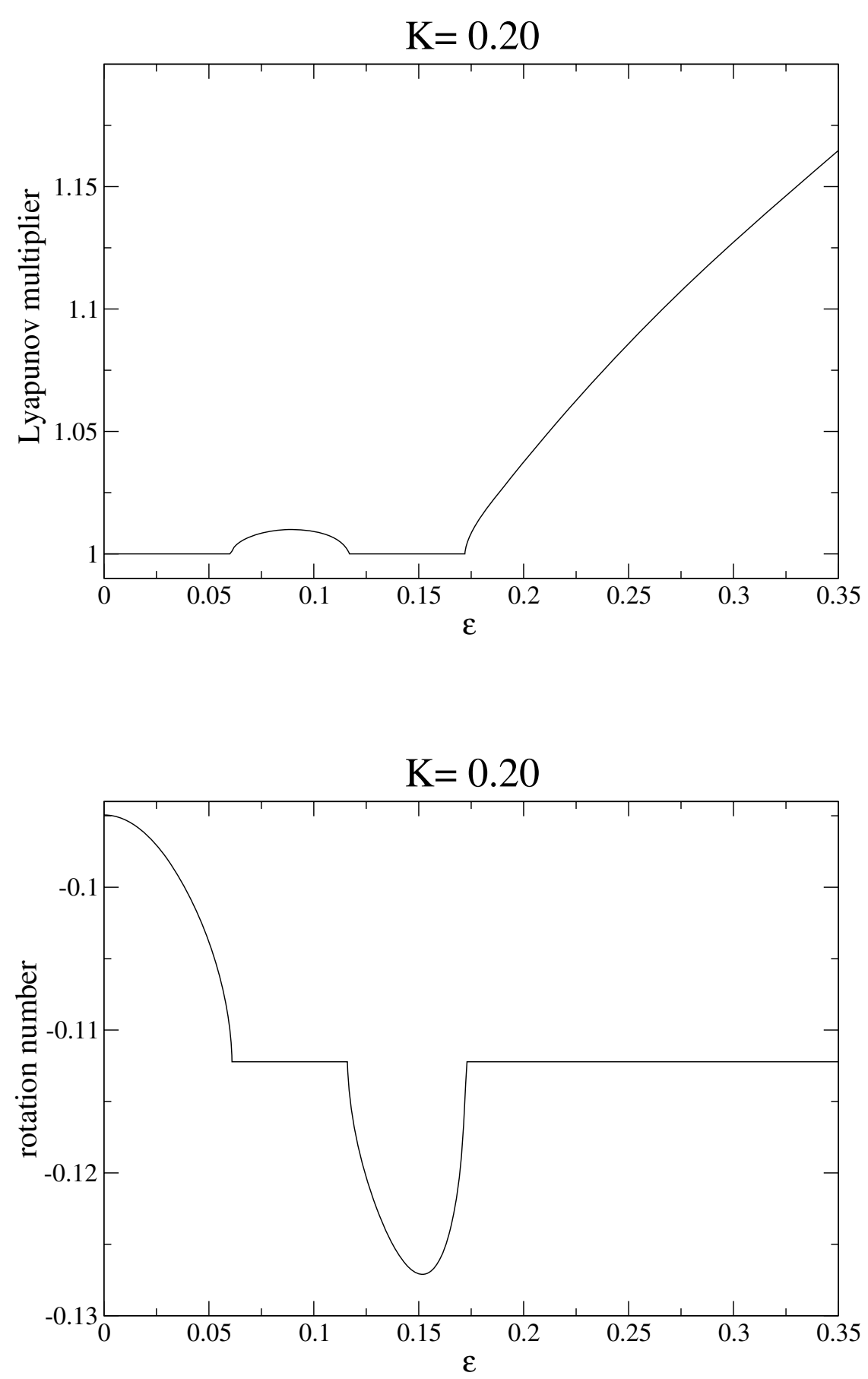

Figure 14. Lyapunov multiplier and rotation number of the cocycle associated to a 3-periodic torus for the rotating standard map, with $K=0.2$. 

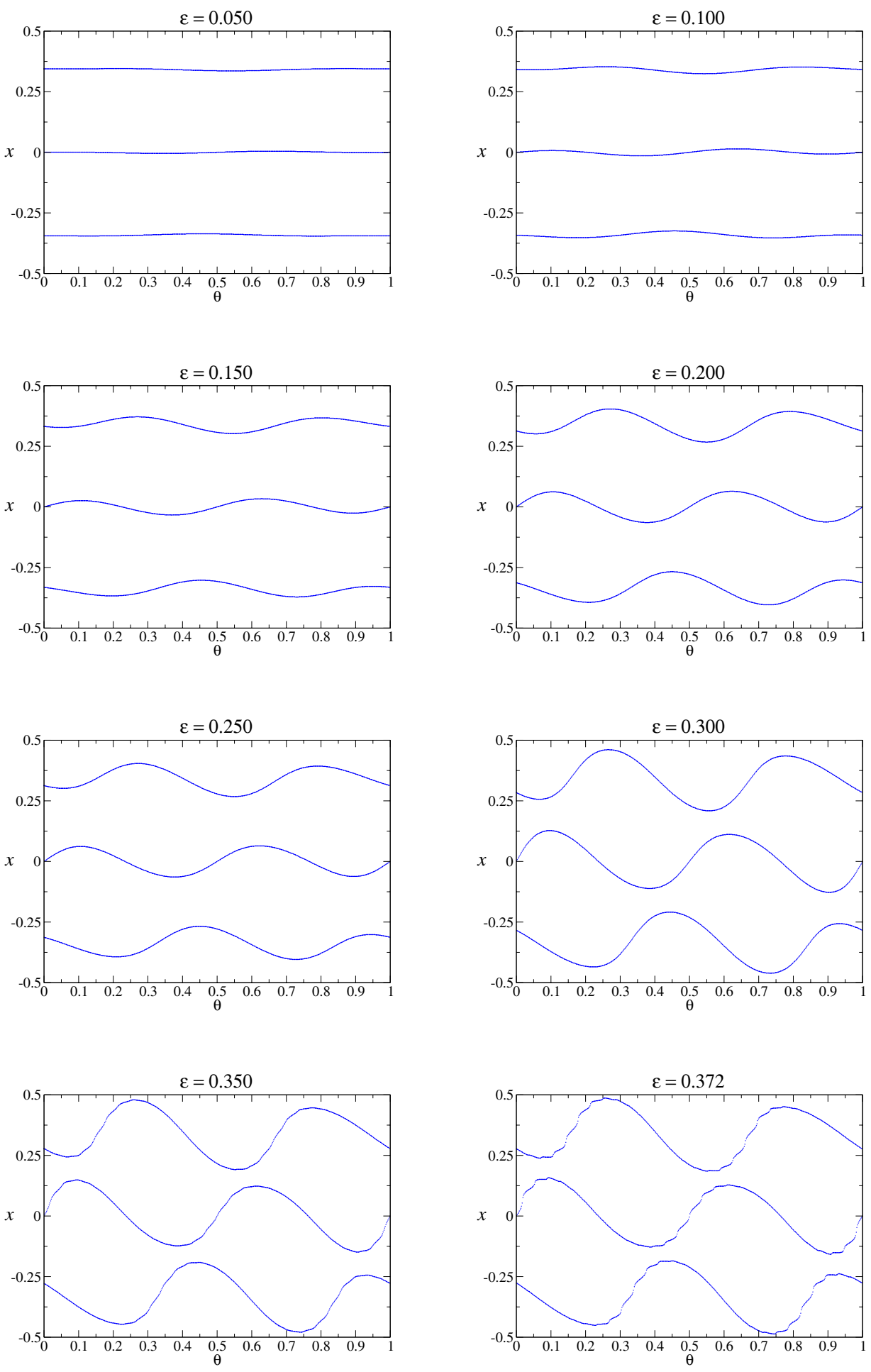

Figure 15. $\theta x$ projection of a 3-periodic curve of the rotating standard map, $K=0.2$. 

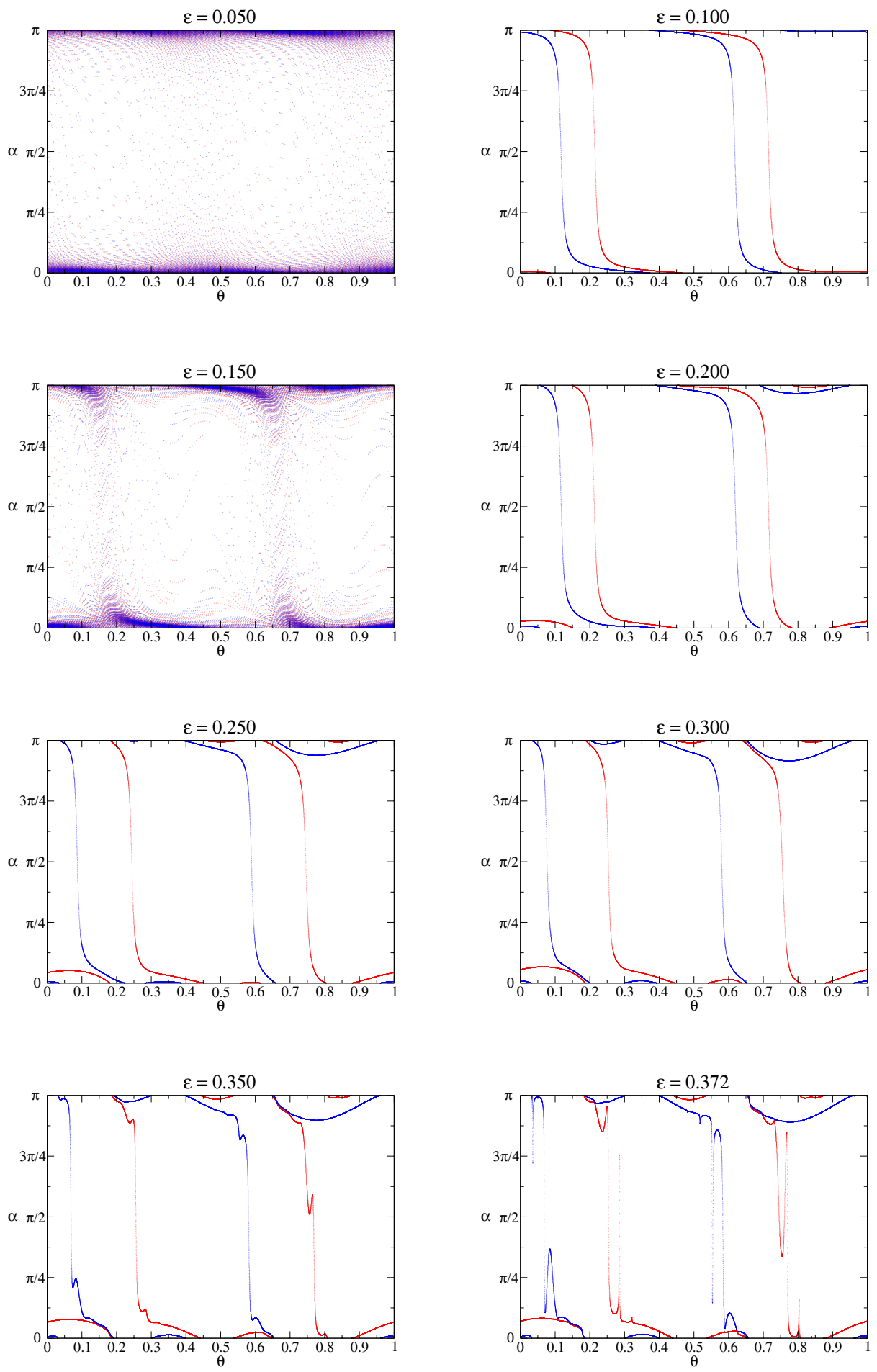

Figure 16. Attractor (red) and repellor (blue) of the projective cocycle associated to a 3-periodic torus of the rotating standard map, $K=0.2$. 
circle. But the unstable and the stable subbundles get closer and closer when increasing the parameter $\varepsilon$.

We have been able to continue the torus and its reducibility until $\varepsilon=0.339$. Since the bundles start to fold a lot, they have to be approximated by Fourier expansions with about 10000 harmonics. Notice, however, that it suffices to use 250 harmonics to approximate the invariant torus.

So, in this regime we have changed to use the full-matrix Newton method. We have continued the torus until $\varepsilon=0.370$, and the distance between the attractor and repellor of the projective cocycle is of the order of thousandths (see Figure 16). However, the Lyapunov multipliers remain far away from 1.

We can again presume that the stable and unstable bundles touch at a critical value near $\varepsilon=0.370$, implying loss of reducibility. See Figure 15 for $\varepsilon=0.370$.

The difference from the example in section 3.2 is that in this case, the spectrum at the collapse of the bundles is a fat annulus that contains the unit circle. So, certainly, the torus is not normally hyperbolic. The theory of [Mn78] suggests that we should expect that the torus breaks down. Certainly, we have found it impossible to continue the Newton method.

So, the continuation reaches $\varepsilon=0.377950$, where the discretization of the torus has 1200 Fourier harmonics, and the invariance equation is solved up to an error $\simeq 10^{-9}$ (notice that the large matrix in the Newton step is about 264 Mbytes). The torus looks rather irregular and like it is about to break. See Figure 23. Moreover, the stable and unstable bundles are extremely close. A more detailed study of the phenomenon pointed out here will be undertaken in section 6 .

In this example, since the torus seems to be smooth and reducible all the way to breakdown, it is possible to make reliable computations reasonably close to breakdown. Recall that a saddle torus is as smooth as the system [HdlL06b], and in this case it is analytic.

Notice, however, that the reducibility method fails because the bundles become so folded that their approximations require very high order Fourier expansions. In a sense, reducibility is much more sensitive to breakdown than the torus itself.

5. Example 3: A Lipschitz rotating standard map. In this section we present an example in which the use of the reducibility method or the projection method is mandatory to compute invariant tori with a high precision.

The example is a quasi-periodic perturbation of the standard map which is Lipschitz but not $C^{1}$. From the numerical point of view, the interest of this map is that the Fourier coefficients of the parameterization of the torus decrease rather slowly with the index, and it is, therefore, a good stress test for the efficiency of the methods. It can be considered as a stand-in for the computation of higher dimensional invariant tori (these computations are actively pursued).

The model we consider is similar to the rotating standard map (27), but the perturbation is given by the 1-periodic and even function defined by

$$
c(\theta)=\left\{\begin{array}{l}
1+4 \theta \text { if }-\frac{1}{2} \leq \theta \leq 0 \\
1-4 \theta \text { if } 0 \leq \theta \leq \frac{1}{2}
\end{array}\right.
$$


in the interval $\left[-\frac{1}{2}, \frac{1}{2}\right]$. The Fourier series of this Lipschitz function is

$$
c(\theta)=\frac{8}{\pi^{2}} \sum_{m \geq 0} \frac{1}{(2 m+1)^{2}} \cos (2 \pi(2 m+1) \theta) .
$$

Notice that the harmonic $k=2 m+1$ decreases as $\frac{1}{k^{2}}$. Henceforth, to approximate $c=c(\theta)$ by its Fourier series with an error smaller than, say, $10^{-8}$, we need an expansion of at least $10^{4}$ harmonics. This means that to compute the invariant tori with this error, we need, at least, to compute $4 \cdot 10^{4}$ coefficients.

The Newton method involves then solving linear systems of such a size at each step. Using quadruple precision, the size of the real variables is 12 bytes, and a matrix of such a size is about 18 Gbytes. So, the direct application of the Newton method is not feasible with a personal computer from 2004.

The reducibility method involves small divisor equations, whose justification needs some regularity to be solved at each step. Nevertheless, we can empirically use the numerical algorithm of reducibility even in regions where the mathematical justification is not yet available. We will obtain approximations of the stable and unstable subbundles that can be validated by the rigorous results in [HdlL06b]. This is one example of a situation where these validation results can be used to justify a posteriori numerical results obtained by algorithms that, by themselves, do not have proofs of convergence.

We have again continued the saddle-type 2-periodic point of the standard map with $K=$ 0.2 into a saddle-type invariant torus, when coupling with $\theta$. The results are displayed in Table 5 and Figure 17. In the computations we have used Fourier expansions with 5000 harmonics.

In order to check how the Fourier coefficients of the curve decrease, we have refined the torus corresponding to $\varepsilon=0.2$, computing the first 100000 harmonics. The harmonics decrease of course very slowly, as can be checked in Figure 18. In fact, they decrease like $\frac{1}{k^{2}}$, as should be expected. Notice also the fine structure of the Fourier spectrum and its organization in curved stripes. We have no explanation for this curious phenomenon, nor any guess on its relevance.

\section{The bundle merging scenario for breakdown of hyperbolicity and exponential di- chotomies.}

6.1. The bundle merging scenario and its consequences. In our previous studies of the rotating Hénon map (see section 3.2) and the rotating standard map (see section 4.5) we have encountered a possible scenario for the disappearance of exponential dichotomies and, in particular, for the disappearance of normal hyperbolicity. In this section, we will investigate the bundle merging in more detail and report some numerical regularities observed. In particular, we will report that there are scaling laws with universal exponents. These scaling relations were already reported in [HdlL06a]. We also mention the paper [JO05] which qualitatively observes situations where the invariant bundles approach each other.

In the bundle merging scenario, the Lyapunov multipliers of the cocycle of the torus remain different, but the bundles corresponding to them approach each other. One can conjecture that, at a critical value, the separation between the invariant bundles disappears and the collision is produced in a dense set of points. 


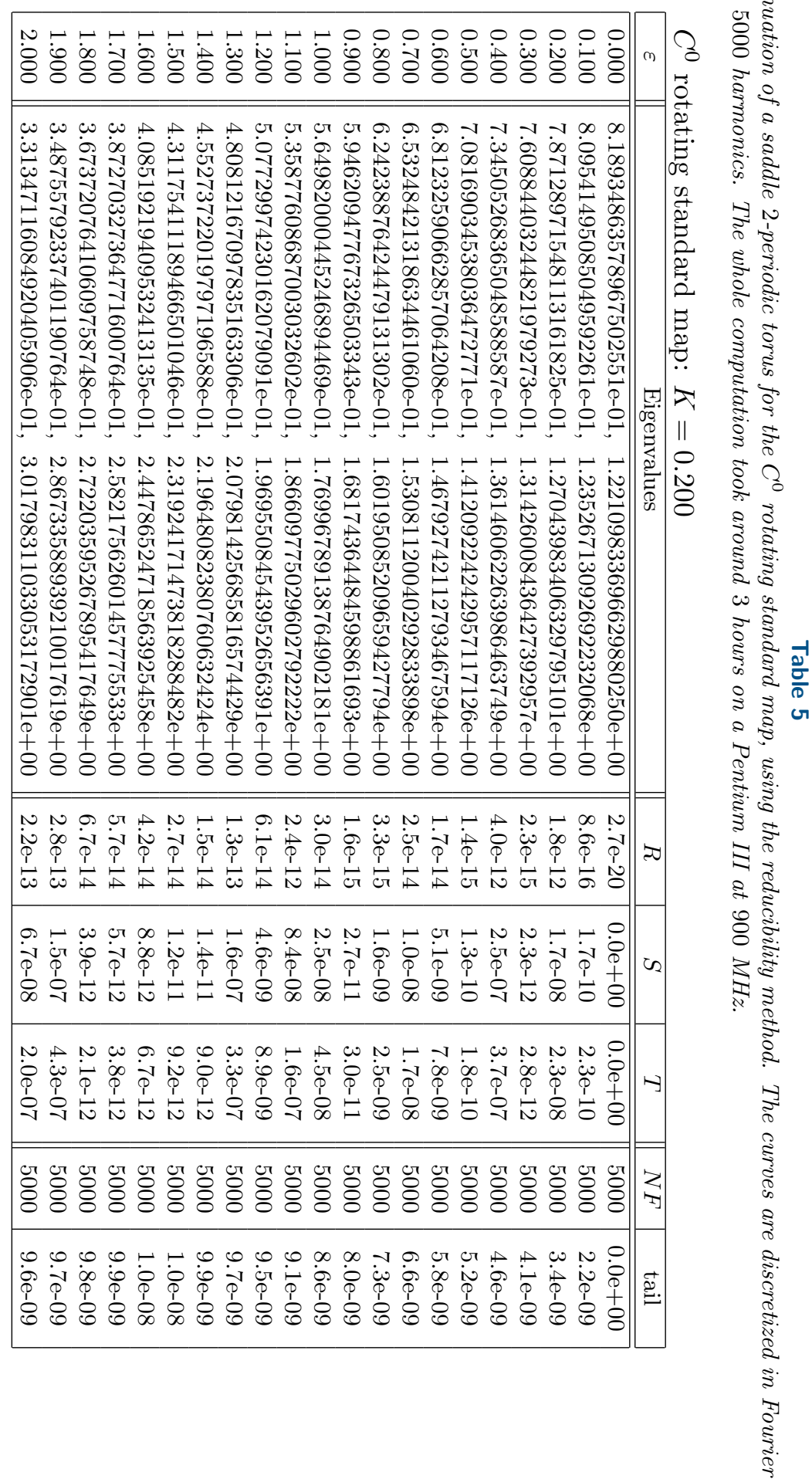



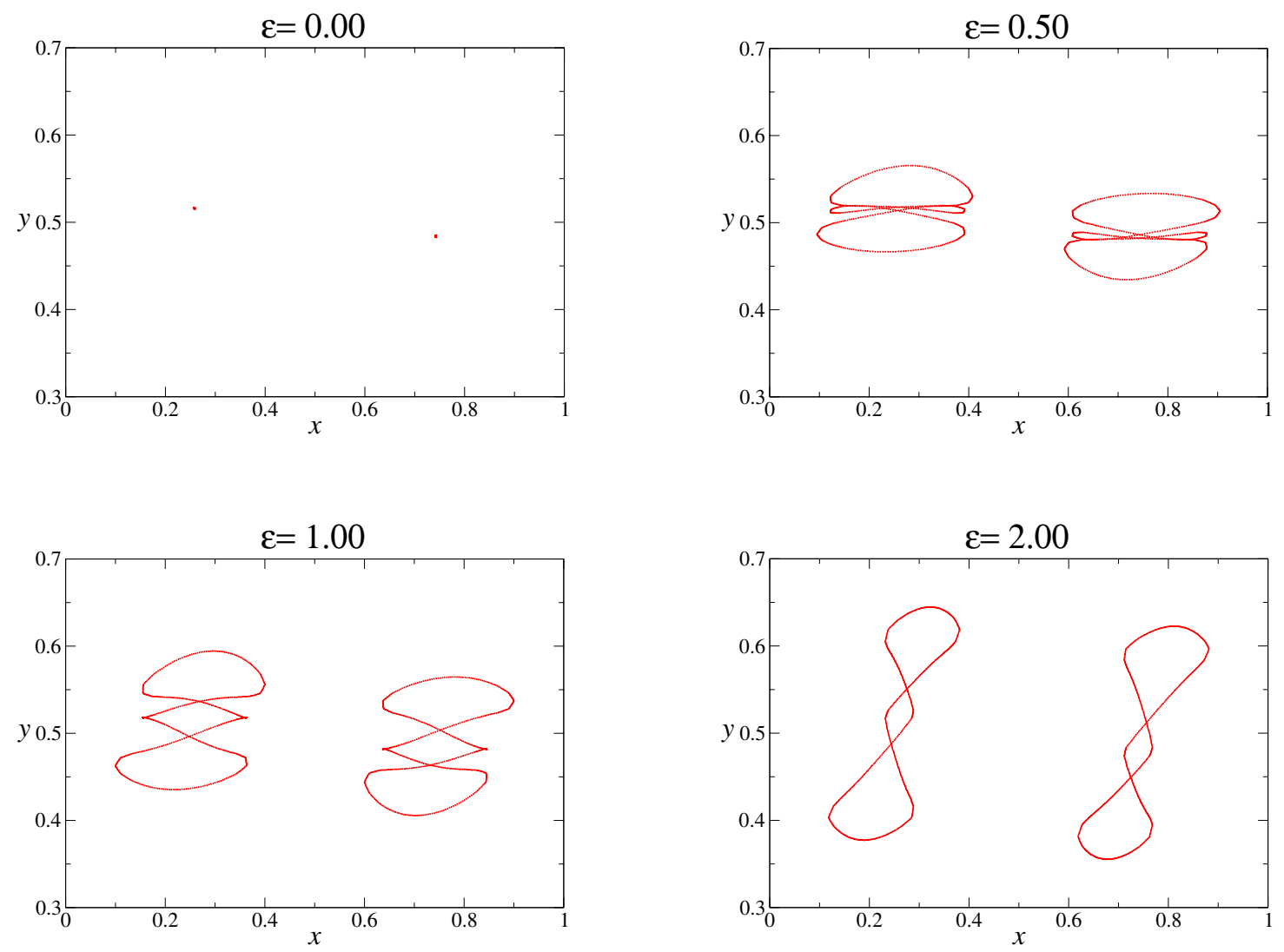

Figure 17. Continuation of the 2-periodic saddle curve for the $C^{0}$ rotating standard map, $K=0.20$. Projection on the xy plane.

Note that the bundle merging scenario implies several consequences.

- Up to the critical value we have the following:

- The maximal and minimal Lyapunov multipliers are different from each other and from 1.

- The spectrum consists of two circles, whose radii are the Lyapunov multipliers.

- The bundles associated to these circles are 1D because we are working with $2 \mathrm{D}$ maps.

- The bundles are as smooth as the cocycle; in this case they are analytic.

- As a consequence, for the examples considered (in which the external rotation $\omega$ is Diophantine), linearization is reducible to constants.

- As the parameter approaches the critical value, the following occur:

- The Lyapunov multipliers remain bounded away from each other and from 1.

- The two bundles get closer, and their minimum distance approaches zero.

- As a consequence, the bundles become more and more oscillatory. Even if they remain analytic, the first derivatives become unbounded. 


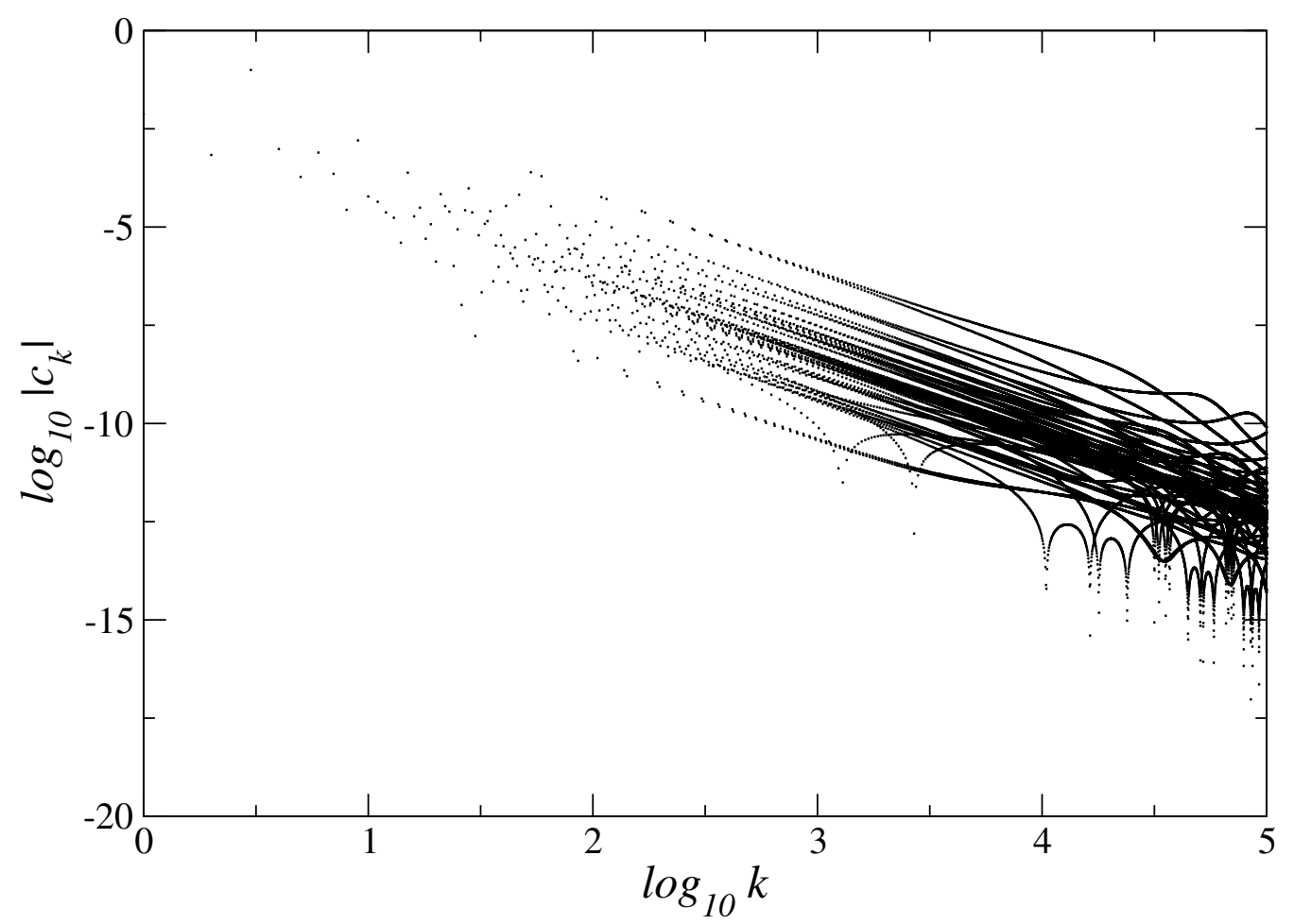

Figure 18. log-log table of the 100000 Fourier modes of the $x$-component of the saddle-type 2-periodic curve for the $C^{0}$ rotating standard map, with $K=0.2, \varepsilon=0.2$. The errors are $R=3.9 e-14, S=1.5 e-11$, $T=1.3 e-11$.

- Even if the spectrum of the transfer operator remains being circles that are far apart, the spectral projections over the corresponding spectral subspaces have norms that become unbounded. (Note that the norm of the spectral projections is closely related to the minimum distance between the bundles.)

- At the breakdown and afterward, it is impossible to discern any continuous invariant bundle.

- As a consequence, the spectrum of the transfer operator at the critical value (and afterward) consists of a filled annulus. The inner and outer boundaries are circles whose radii are the Lyapunov multipliers.

- The invariant bundles are not continuous. The Oseledec theorem [Ose68] asserts that the bundles are defined in a set of full measure, and they are measurable.

Remark 6.1. At first sight it may seem paradoxical that the bundles approach each other at some points but the Lyapunov multipliers remain different.

It is certainly true that, when the bundles are very close at some point, the Lyapunov multipliers at a fixed time become very close. Nevertheless, remember that the Lyapunov multipliers are obtained by taking the limit of the number of iterations tending to $\infty$ so that 
the proximity for finite time does not imply the proximity of the limits. (In other words, we cannot exchange the limits of the parameters or the $n \rightarrow \infty$ in the expresion of the time $n$ Lyapunov exponents.)

Note that the formula (16) is affected little by intervals of small length in which the integrand takes the "wrong" value.

It is true that the computation of the Lyapunov exponent requires longer and longer times to achieve a certain accuracy as we approach the critical value.

Remark 6.2. At the critical value of the parameter, the spectrum of the transfer operator experiences a sudden growth and it is discontinuous as a set. It is well known that the spectrum cannot suddenly decrease [Kat76], but in the same reference, we can find other examples with sudden growth. This phenomenon of sudden growth of the spectrum is closely related to the fact that the spectral projections become unbounded.

Remark 6.3. Notice also that, even if Oseledec bundles are not continuous at a critical value, some kind of regularity could be expected (e.g., Whitney differentiability). This is perhaps what is observed in Figures 5 and 6 (see also Figures 20 and 21). For studies on the regularity of Oseledec bundles, see [Pes77], and for regularity of invariant graphs on forced systems (that here correspond to the projectivization of invariant bundles), see [Sta99].

6.2. Quantitative regularities of bundle collapse. The observables we consider to measure the bundle collapse are the minimum distance between the bundles $(\Delta)$, which goes to zero at the collapse for $\varepsilon=\varepsilon_{c}$, and the upper and lower Lyapunov multipliers $\left(\Lambda^{ \pm}\right)$, which go to critical values $\Lambda^{ \pm}$when $\varepsilon$ goes to $\varepsilon_{c}$.

The most remarkable fact about this transition is that there seem to be some scaling relations of these observables with exponents that are not affected by changes in the map.

More precisely, we formulate the following assertion.

Assertion 6.4. For an open set of families $f_{\varepsilon}$, we have the following.

The observables $\Delta$ and $\Lambda^{ \pm}$satisfy the asymptotics

$$
\Delta_{\varepsilon} \sim \alpha\left(\varepsilon_{c}-\varepsilon\right)^{\beta}, \quad \Lambda_{\varepsilon}^{ \pm} \sim \Lambda_{c}^{ \pm}+A^{ \pm}\left(\varepsilon_{c}-\varepsilon\right)^{B}, \quad \varepsilon \lesssim \varepsilon_{c},
$$

where $\alpha, \beta, A^{ \pm}, B$ (and $\Lambda_{c}^{ \pm}$) are fitting parameters.

We distinguish two cases:

(a) The Lyapunov multipliers do not straddle $1: \beta=1, B=0.5$.

(b) The Lyapunov multipliers straddle 1: $\beta=1, B=1$.

We emphasize that in case (b) above the torus is (generically) destroyed, because the stable and unstable bundles collapse. In case (a), if the torus is attracting, it survives after the collapse of the fast and slow stable bundles in the critical value $\varepsilon_{c}$. Then, we can also make estimates of the observables after this critical value. Our numerical computations suggest the following.

Assertion 6.5. For an open set of families $f_{\varepsilon}$ in case (a) above, we also have the approximations

$$
\Delta_{\varepsilon} \approx 0, \quad \Lambda_{\varepsilon}^{ \pm} \approx \Lambda_{c}^{ \pm}+\bar{A}^{ \pm}\left(\varepsilon-\varepsilon_{c}\right)^{\bar{B}}, \quad \varepsilon \gtrsim \varepsilon_{c}
$$

where $\bar{B}=1$. 
Remark 6.6. For comparison, we recall that known bifurcations - which have analogues for quasi-periodic tori-have scaling relations. See [BHTB90]. The saddle node has $\beta=0.5$, $B=0.5, \Lambda_{c}^{ \pm}=1$, and the transcritical has $\beta=1, B=1, \Lambda_{c}^{ \pm}=1$.

Notice, however, that, in the bifurcations considered in [BHTB90], the bundles remain smooth. This is very different from what happens in the problems considered here.

Remark 6.7. We already noted that if the Lyapunov multipliers, whose associated bundles collapse, do not straddle 1, then the torus continues being normally hyperbolic and survives after the collapse. (In the example presented here, the torus is attracting. See section 3.)

If the Lyapunov multipliers do straddle 1, the torus is not normally hyperbolic at collapse, and a generic perturbation destroys the torus [Mn78]. (See section 4.5.)

Hence, there is an important difference between both cases, which explains the different scalings found.

Notice, however, that one can create an example in which a saddle torus survives after the bundle collapse because the perturbation vanishes on the torus. (See section 4.3.) In this case we found the scalings to be those in (a) of Assertion 6.4.

Remark 6.8. While Assertion 6.4 conjectures asymptotic formulas for the observables, Assertion 6.5 gives only approximations. We also note that the numerics of Assertion 6.4 can be validated using the well-developed theory of normally hyperbolic systems.

Notice, however, that it is not easy to assess the reliability of the calculations of Assertion 6.5, since the exponential dichotomies have been broken. It seems quite possible that there are gaps of parameter values where $\Delta$, even smaller than the precision of the present computation, is nevertheless positive. A "big" gap $\left[\varepsilon_{\mathrm{c}}, \varepsilon_{\mathrm{d}}\right]$ has been already observed in section 3.1. See section 6.3.4 for other "small" gaps. One can conjecture that the fact that increasing the precision reveals more structure will continue. This is consistent with the mathematical work in [AK03].

6.2.1. Results for the rotating Hénon map. As evidence for Assertions 6.4 and 6.5, we present some more detailed computations done in the quasi-periodic Hénon map (25), with $a=0.68, b=0.1$, and $\omega=(\sqrt{5}-1) / 2$. Similar computations have been carried out for some modifications of the map (changing parameters, adding terms, and even taking other rotations-for example, $\omega=e / 4)$.

Below is a description of the results of detailed calculations at the bifurcation (b) described in section 3.1. Similar calculations have been performed for the bifurcations (c) and (d) in section 3.1.

We have computed the observables $\Delta$-minimum distance between the bundles - and $\Lambda$ maximum Lyapunov multiplier - of the attracting circle for values of $\varepsilon$ in [0.463240, 0.463270], and fit parameters in (33), (34). We note that, in this case, since the circle is an attractor, the computation can be done without using Fourier methods. It suffices to iterate the map to compute the attractor up to round-off error precision. Once we have the attractor, we iterate the projectivized version of the linearization (15) both forward and backward as indicated in section 2.2.1.

We note that, in this case, the torus, being a uniform attractor, remains very smooth (in fact, as smooth as the system, which in our case implies that the torus is analytic). The calculation is done always in the situation where the torus is smooth and the calculation is quite reliable. Then, the values are extrapolated. 

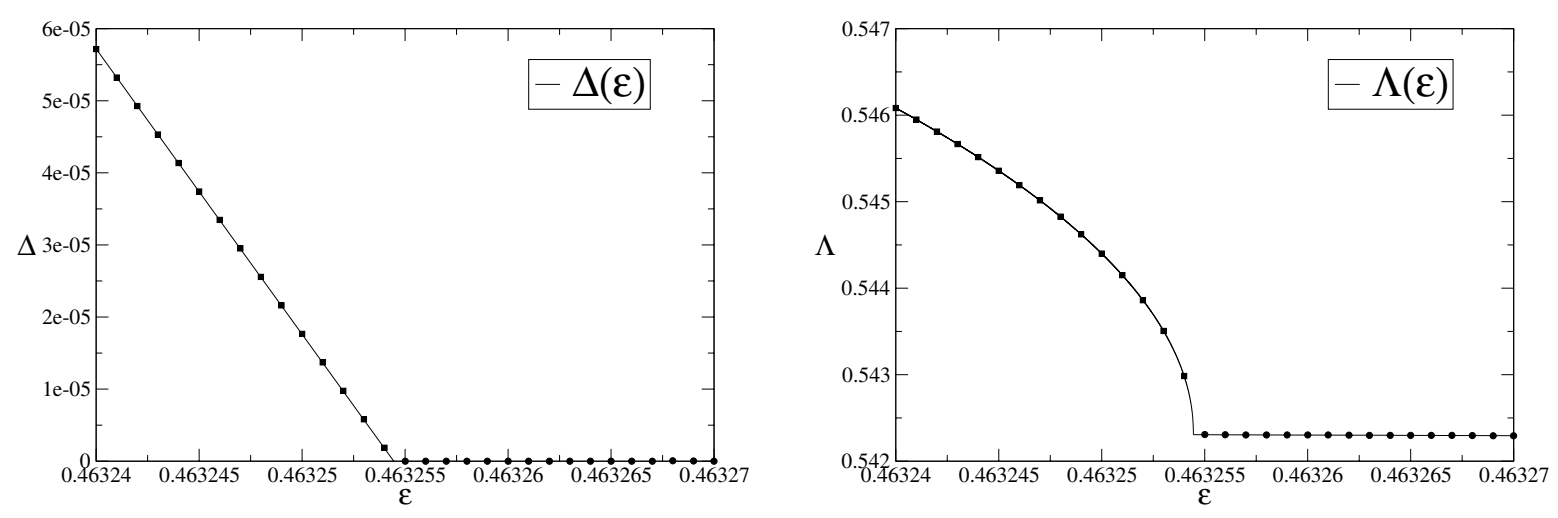

$$
\begin{aligned}
& \varepsilon_{c}=0.46325447112 \pm 1 \cdot 10^{-11} \\
& \alpha=3.94933 \pm 7 \cdot 10^{-5} \\
& \beta=0.999979 \pm 2 \cdot 10^{-6}
\end{aligned}
$$

$$
\begin{aligned}
& \Lambda_{c}=0.5423122 \pm 5 \cdot 10^{-7} \\
& A=1.015 \pm 1 \cdot 10^{-3} \\
& B=0.5020 \pm 1 \cdot 10^{-4} \\
& \bar{A}=-0.7409 \pm 6 \cdot 10^{-4} \\
& \bar{B}=1.00035 \pm 8 \cdot 10^{-5}
\end{aligned}
$$

Figure 19. Fits of $\Delta$ and $\Lambda$ to (33), (34) for the transition $\mathrm{b}$ that is produced between $\varepsilon=0.463254$ and $\varepsilon=0.463255$.

Table 6

Maximal Lyapunov multiplier of the attractor of the rotating Hénon map, and estimate of the error, for different values of $\varepsilon$ close to the critical value $\varepsilon_{c}=\varepsilon_{\mathrm{b}}$. The computations have been done by direct iteration of the cocycle on a vector 701408733 times, after a transient time of 100000 steps.

\begin{tabular}{|c|c|c|}
\hline$\varepsilon$ & $\Lambda$ & Error \\
\hline \hline 0.4632544710 & 0.5423194946536124 & $1.0 \mathrm{e}-13$ \\
0.4632544711 & 0.5423149288182757 & $1.0 \mathrm{e}-13$ \\
0.4632544712 & 0.5423065545078773 & $5.2 \mathrm{e}-10$ \\
0.4632544713 & 0.5423065555707195 & $5.8 \mathrm{e}-10$ \\
0.4632544714 & 0.5423065550474250 & $1.0 \mathrm{e}-09$ \\
\hline
\end{tabular}

The results of the fits are depicted in Figure 19. Notice that we can extrapolate the value of the transition: $\varepsilon_{\mathrm{b}} \simeq 0.4632544711$. See Table 6 for further numerical evidence, that is, the change of the accuracy in the computation of the Lyapunov multipliers. The attractors and their bundles are represented in Figure 20. A magnification of the invariant bundles right before collapse is depicted in Figure 21. Note that in Figure 21 we find a significant difference by changing the parameter value of $\varepsilon$ by just $10^{-6}$.

Among many other verifications of universality we have carried out, we present in Figure 22 an analogue of Figure 19 when $\omega=e / 4$. Note that even if $e / 4$ is numerically close to $(\sqrt{5}-1) / 2$, its number theoretical properties are very different.

6.2.2. Results for the rotating standard map. We now discuss the phenomena that are observed for the rotating standard map (27). The situation is somewhat different from the situation discussed previously. One obvious difference is that the map is area preserving. Also, since at collapse the spectrum of the linearization is a fat annulus that includes 1 , we expect 

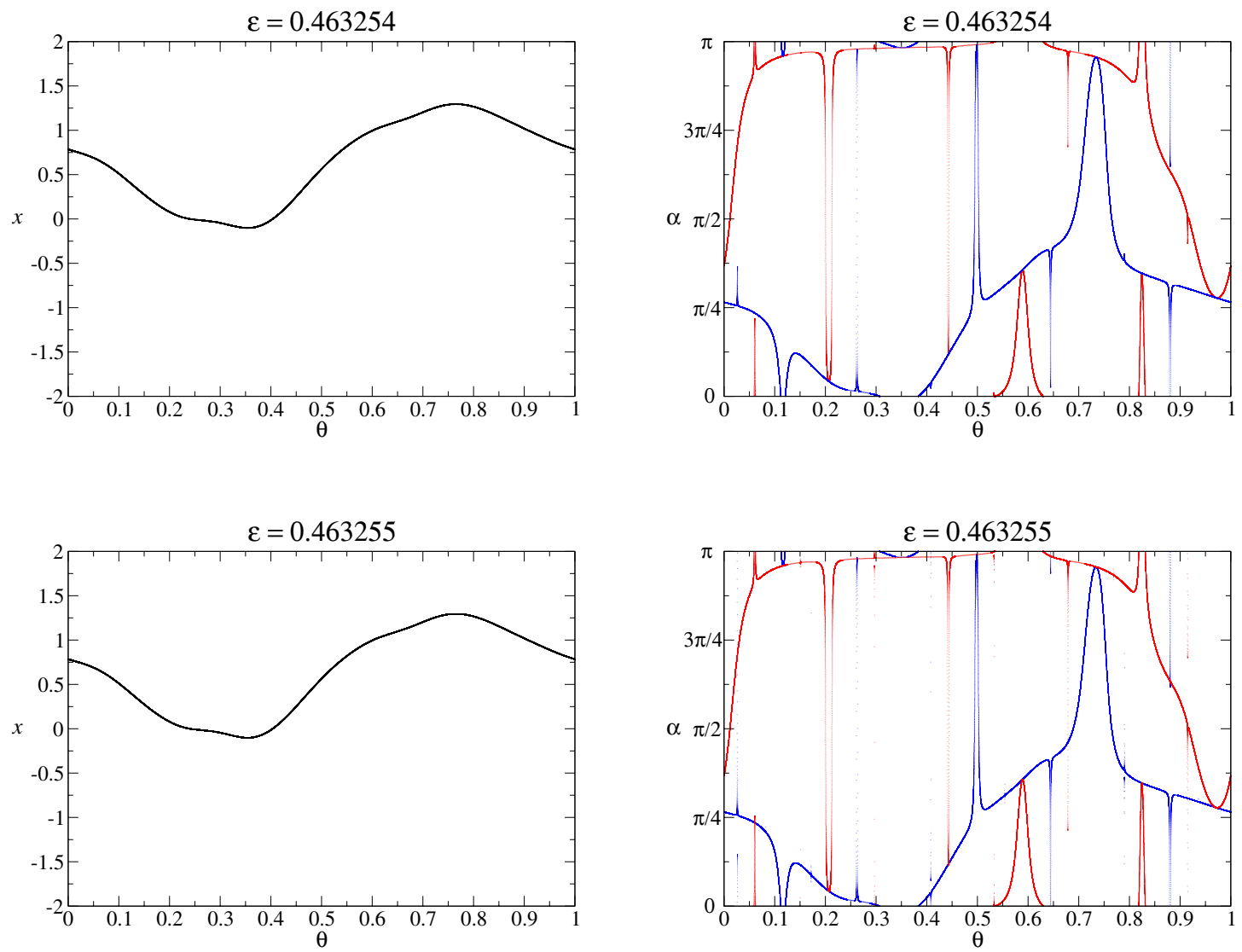

Figure 20. $x$-curve of an attracting torus and $\alpha$-curves of its slow and fast directions, before and after their collapse.

that the invariant circle disappears at collapse.

In section 4.5 we have continued with respect to $\varepsilon$ a 3 -periodic torus. The torus becomes hyperbolic at $\varepsilon \simeq 0.173$ and remains hyperbolic for larger values of $\varepsilon$.

The continuation reaches $\varepsilon=0.377950$, where the torus looks rather irregular and about to break, and the stable and unstable directions are extremely close. See Figure 23. Hence, we conjecture that this is another case of bundle collapse. Of course, the irregularity of the torus is in marked contrast with the situation for the rotating Hénon map discussed before.

We can explore the torus for values of $\varepsilon$ close to this value. The results of fitting the scaling laws in Assertion 6.4 are depicted in Figure 24. Notice that we produce an estimate of the critical value in which the torus is destroyed: $\varepsilon_{c} \simeq 0.379696$.

Compared with the phenomena studied in the rotating Hénon map, we find several differences. The most notable one is that the torus does not seem to persist (at least as a smooth curve). Hence, there is no scaling relation after the bundle merging. Also we note that the torus becomes more irregular.

From the computational point of view, since the projection and reducibility methods 

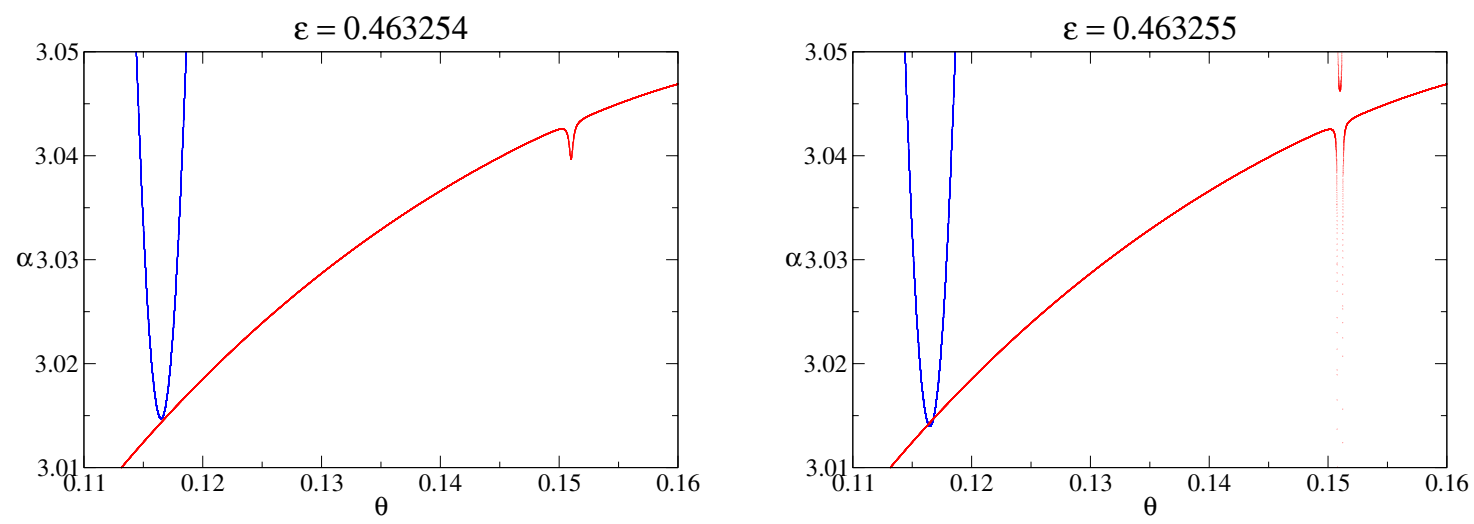

Figure 21. Zooms of the $\alpha$-curves of Figure 20.
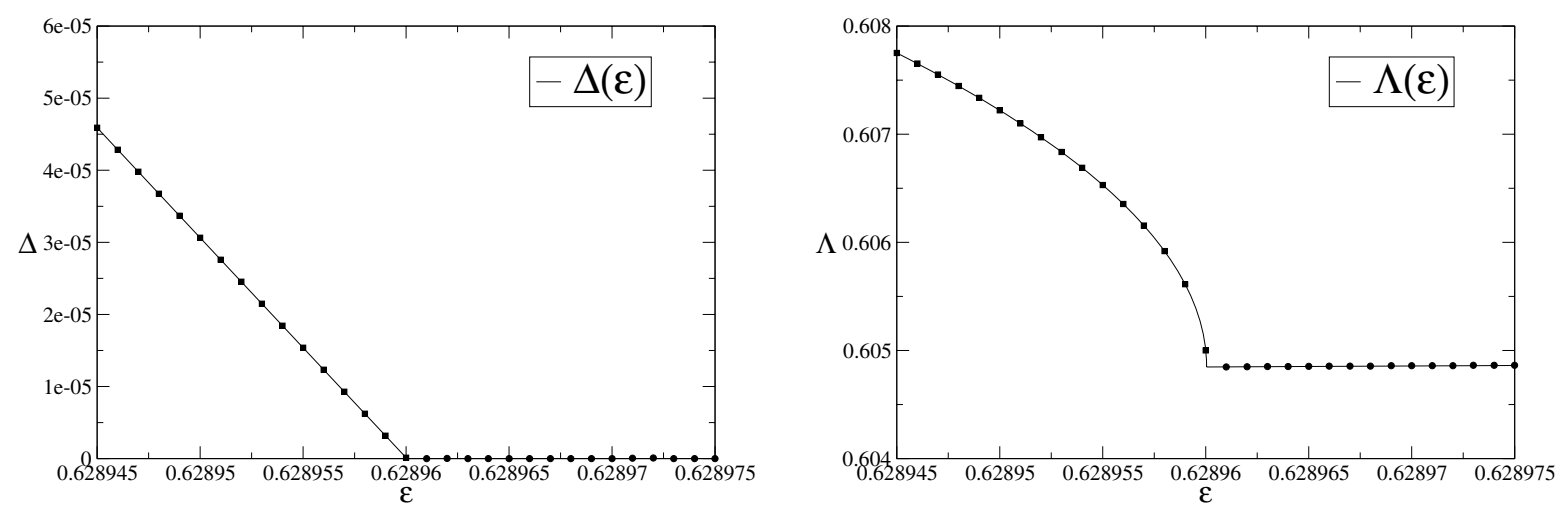

$$
\begin{aligned}
& \varepsilon_{c}=0.62896004195 \pm 5 \cdot 10^{-11} \\
& \alpha=3.0546 \pm 3 \cdot 10^{-4} \\
& \beta=1.000098 \pm 9 \cdot 10^{-6}
\end{aligned}
$$

$$
\begin{aligned}
& \Lambda_{c}=0.60484735 \pm 1 \cdot 10^{-8} \\
& A=0.7428 \pm 3 \cdot 10^{-4} \\
& B=0.4993 \pm 4 \cdot 10^{-5} \\
& \bar{A}=0.93 \pm 9 \cdot 10^{-2} \\
& \bar{B}=0.983 \pm 8 \cdot 10^{-3}
\end{aligned}
$$

Figure 22. Fits of $\Delta$ and $\Lambda$ to (33), (34) for a bundle merging bifurcation in an attracting torus of the rotating Hénon map with frequency $\omega=e / 4$, and $a=0.68, b=0.1$. The transition is produced between $\varepsilon=0.628960$ and $\varepsilon=0.628961$.

become more problematic as we approach the critical value, we have to use the full-matrix Newton method. Simple iteration is not possible because the torus is not attracting. As a consequence, we can only explore values of $\varepsilon$ which are farther apart from the critical value than those allowed in the rotating Hénon map.

6.3. Some open questions. We conclude this article by mentioning several open questions that arise from the computations here. 

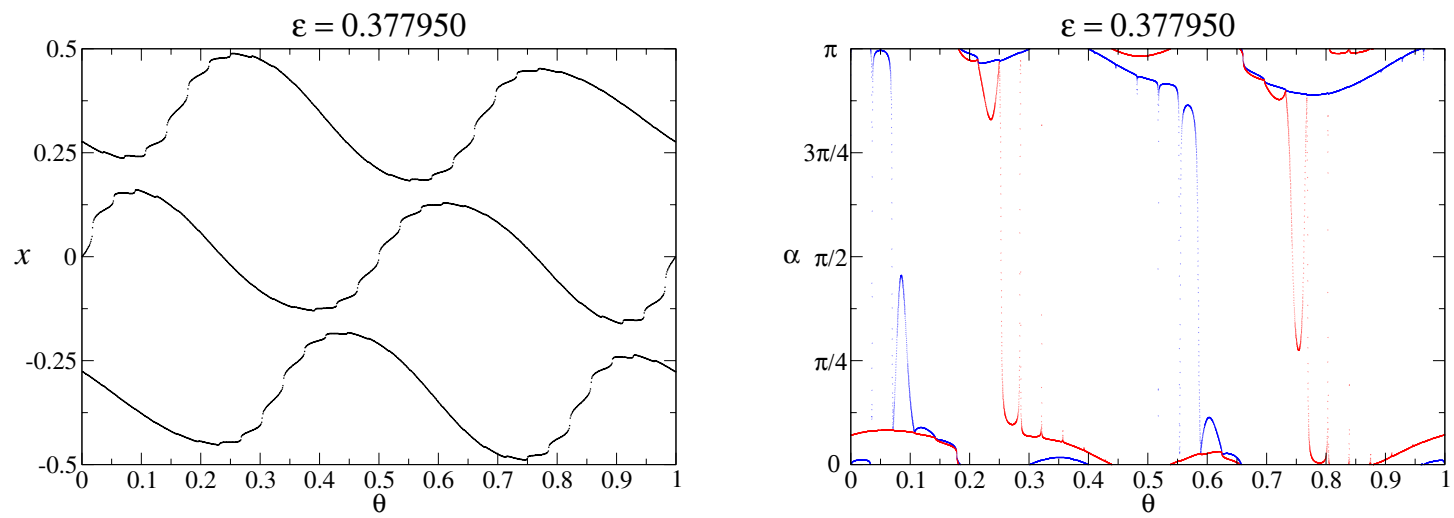

Figure 23. $x$-curves of a 3-torus close to breakdown, and the $\alpha$-curves of its unstable and stable directions.
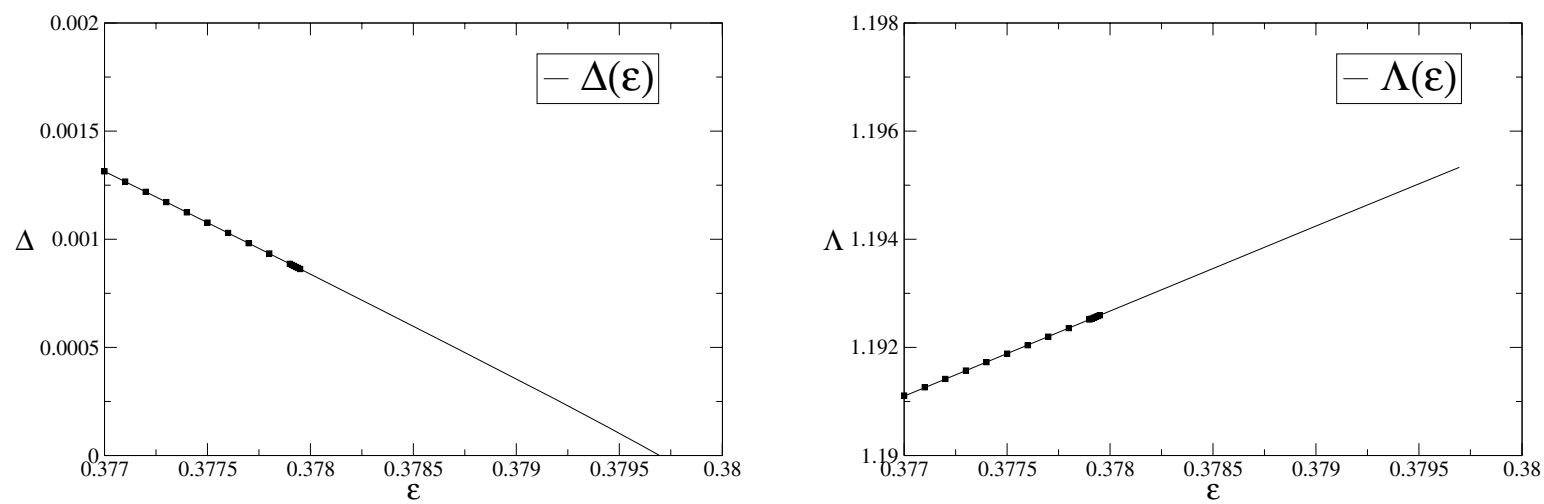

$$
\begin{aligned}
& \varepsilon_{c}=0.3796965 \pm 8 \cdot 10^{-7} \\
& \alpha=0.4063 \pm 7 \cdot 10^{-4} \\
& \beta=0.9693 \pm 4 \cdot 10^{-4}
\end{aligned}
$$

$$
\begin{aligned}
& \Lambda_{c}=1.19533 \pm 9 \cdot 10^{-5} \\
& A=-1.6 \pm 2 \cdot 10^{-1} \\
& B=1.00 \pm 3 \cdot 10^{-2}
\end{aligned}
$$

Figure 24. Fits of $\Delta$ and $\Lambda$ to (33) near breakdown.

6.3.1. A renormalization group description. The existence of the scaling properties above suggests that there could be a renormalization group explanation for the phenomena happening. Unfortunately, we have not been able to find a renormalization group transformation which has a fixed point which explains the behavior observed. Nevertheless we point out that renormalization of quasi-periodic cocycles has been considered in the literature in somewhat different contexts. For example, renormalization of Schödinger quasi-periodic cocyles appears already in [Hof76]. Renormalization of quasi-periodic maps has been considered in [OM03, MO00, MO04]. Renormalization of general cocycles of matrices and its relation with reducibility was considered in [Ryc92]. Renormalization procedures for $2 \mathrm{D}$ cocycles play an important role in [Kri99c, Kri01]. Nevertheless, the conclusions of later renormalizations are 
not formulated in terms of fixed points of an operator and they seem to be in regimes different from the ones considered in this paper.

We think that developing a renormalization group explanation of the phenomena reported here is a very interesting problem. We note, however, that in contrast with other renormalization groups studied, the phenomenon seems to be independent of the rotation number.

6.3.2. Scaling exponents of the observables. The fact that the exponents found numerically are close to being simple numbers makes us hopeful that perhaps there is a relatively simple explanation. Nevertheless, since one has to deal with bundles that are becoming not very smooth it is not clear that a simple normal form explanation works.

We think that exploring whether there is a simple explanation of the scaling behavior reported here is a very interesting problem.

Remark 6.9. The square root behavior of the Lyapunov multiplier has also been observed in a growth of spectrum phenomena similar to that described above for a rotating logistic map that is a quasi-periodically forced noninvertible $1 \mathrm{D}$ map. In this case, the mechanism involves the loss of invertibility of the cocycle of the attractor, and it is possible to do a normal form analysis [JT05].

Besides the scaling properties reported in Assertions 6.4 and 6.5, it seems that there is a host of other scaling properties to be observed in this scenario.

We are actively pursuing these numerical explorations, but it is clear that much more needs to be done.

Of course, besides finding more observables that scale, an interesting problem is to find the domain of universality of these relations.

6.3.3. Explicit models of cocycles exhibiting the bundle merging scenarios. The bundle merging scenario is a property of the projective cocycle. Since the projective cocycle is independent of multiplication of the linear cocycle by a constant, it superficially seems that the distinction between the cocycle straddling 1 or not is meaningless.

On the other hand, we note that when the cocycle straddles 1 , we expect that the torus disappears and that, therefore, as we approach the critical value, the torus becomes more oscillatory. Hence, the difference between the bundles straddling 1 or not is really a difference between the projective cocycles becoming more oscillatory or not.

We think that it would be very interesting to develop explicit models of linear cocycles that exhibit bundle merging without any reference to the underlying invariant torus. Given the above observations, one can expect that the bundle merging with the spectrum straddling 1 will require cocycles with rather irregular matrices.

6.3.4. Abundance of gaps with exponential dichotomy. In the study of the rotating Hénon map, we have encountered regions of the parameter space in which there are slow and fast stable smooth invariant bundles $(\Delta>0$, reducible case) and others in which they are not smooth $(\Delta=0$, nonreducible case). We have also found scaling relations in the transition from one to the other case (see Assertions 6.4 and 6.5).

Notice that $\Delta>0$ holds in open sets of the parameter space. Even if we present numerical evidence for $\Delta \approx 0$ in an open set of the parameter space (see Assertion 6.5), the theory in [AK03] suggests that in fact $\Delta>0$ in small gaps of the parameter space that are missed by 

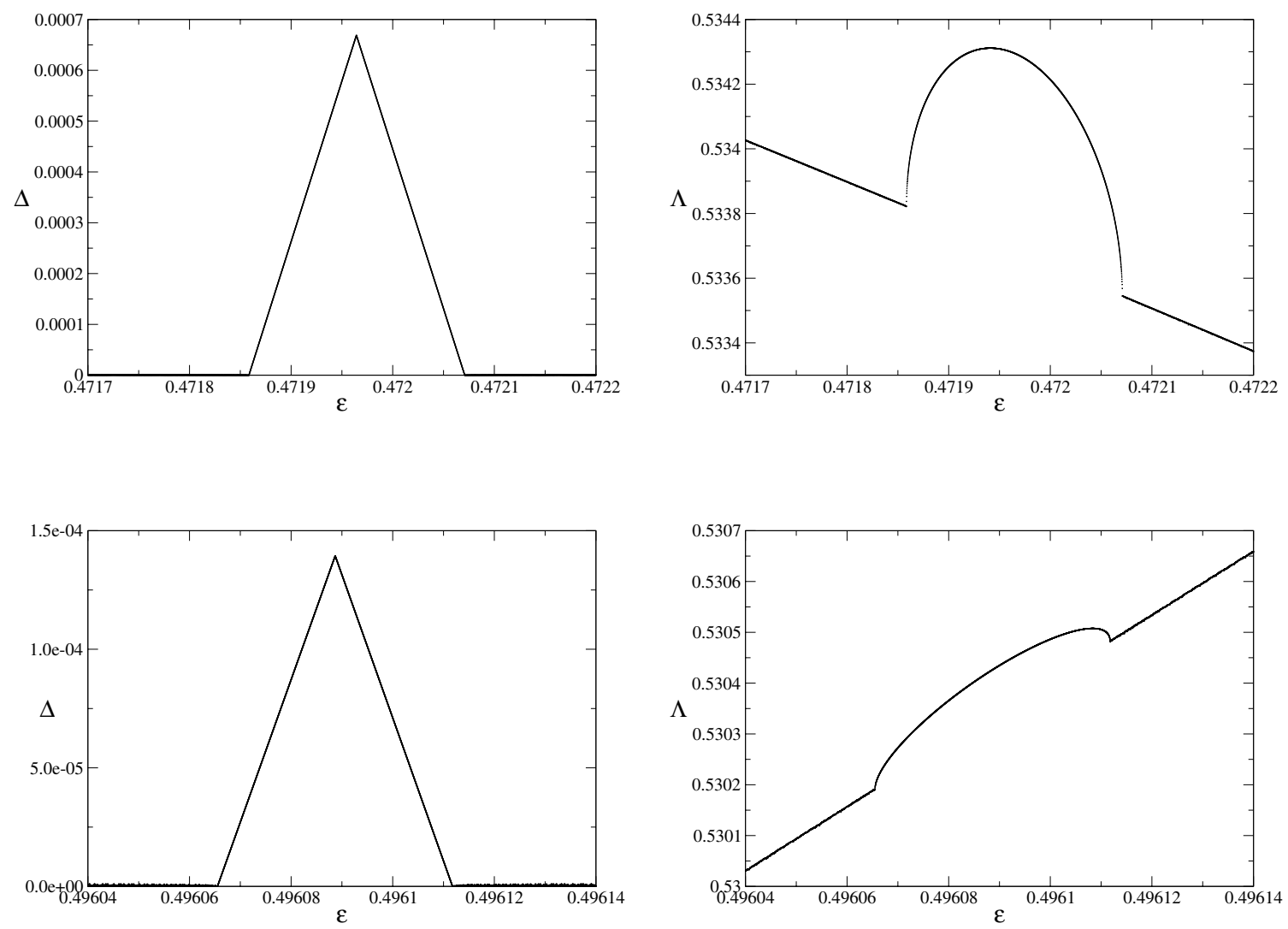

Figure 25. Some small gaps of reducibility. The observables $\Delta$ (left) and $\Lambda$ (right) in two intervals of the parameter $\varepsilon$ for the Hénon map.

the numerical computation. Some of these gaps for the example in section 6.2.1 are shown in Figure 25.

So, it would be very interesting to quantify the abundance of reducibility and nonreducibility in this context. It is quite plausible that reducibility holds in an open and dense subset of parameter space, but its structure can be very complicated. On the other side, nonreducibility could happen in a closed set of positive measure. (See [HP05] for a model in which this picture holds.)

6.3.5. Existence of strange nonchaotic attractors. The global phenomena that happen at $\mathrm{e}$ in section 3.1 are not well understood and they deserve a more detailed study. After the transition e the attractor looks like a strange chaotic attractor (SCA). It seems that this is related to the very poorly understood phenomena of SNAs, and in particular to the so-called fractalization mechanism or route "torus $\rightarrow \mathrm{SNA} \rightarrow \mathrm{SCA}$." (See [PNR01] for a review of experiments in this field. See also [GFPS00, OWGF01].) There are some precise definitions of SNA in the literature. See, for example, [Kan94, Kel96, WY01, WY02, BDV04]. 
The theory in [HdlL06b] and the numerical experiments described here suggest that the fractalization mechanism is in fact a route "torus $\rightarrow \mathrm{SCA}$ " and that in this transition SNAs are not created. Further detailed numerical experiments of this transition confirm this description. (See [BSV05, JT05] for similar studies on the rotating logistic map that is 1D and noninvertible, [HdlL05b] for the rotating Hénon map, and [HS05] for a comprehensive study of both cases.) But, so far, there are not rigorous explanations of the fractalization mechanism.

We also note that, at the critical value of bundle merging, the attractors and repellors for the projectivization look like SNAs, as in the collision mechanism described in the literature [PNR01]. We have obtained evidence in several examples that the collapses are really produced based on topological arguments. Our interpretation is that the SNAs are the projectivization of the Oseledec bundles that are measurable but not continuous after the collapse. It would be quite interesting to decide whether the objects formed at the critical value of the bundlemerging bifurcation fit the rigorous definitions of SNAs. (See [HP05] for the implication of this mechanism in the existence of SNAs in Harper maps.)

Notice also that, in the experiments performed here, both mechanisms seem to be related. That is, the collision mechanisms that happen at the level of the linearization of the dynamics around the torus have a strong influence on the geometric behavior of the torus itself.

6.3.6. Extensions to other dynamical systems. We emphasize that the bundle merging scenario is a dynamical/geometrical/functional mechanism that can be generalized to higher dimensions, to other base dynamics, etc., that is, to general bundle maps (and their invariant sections).

The linearization around an invariant section is a vector bundle map in a suitable vector bundle (the normal bundle to the invariant section).

From the dynamical point of view, the mechanism is the progressive deterioration of the exponential dichotomies in the linearization (the uniform constant $C$ in (20) tends to infinity in the collapse).

From the geometric point of view, it consists in the collapse of the invariant bundles in a nonsmooth way.

From the functional analysis point of view, it consists in a sudden growth of the spectrum of the transfer operator. This functional analysis behavior has different geometric implications depending on whether the rates involved in the collapse straddle 1 or not.

It would be interesting to obtain scaling laws in these transitions for cases other than rotations.

Extensions to fully coupled systems will be also very interesting.

After this paper was submitted, we became aware of [CLR06, CLR]. These papers contain a study of some regions where the Hénon family loses hyperbolicity. They show that the hyperbolicity may be lost even if the Lyapunov exponents of all invariant measures are bounded away from zero. The reason is there are points where the invariant bundles become close to each other. This is reminiscent of the bundle merging scenario discussed here. It would be quite interesting to study whether the rigorous analysis of the above papers applies to the examples here. Conversely, it would be quite interesting to find out whether the quantitative scaling laws found here apply to the internal tangencies. We also became aware of [Bjerklov05, Bjerklov06], which study linear cocycles, find phenomena reminiscent of the 
bundle merging scenario, and are related to [HP05].

Acknowledgments. We thank R. Fabbri, À. Jorba, W. Liu, S. Luzzatto, J. Puig, and C. Simó for several discussions and relevant references. We thank the referee for a very thorough reading which improved the exposition.

\section{REFERENCES}

[ACS91] R. Artuso, G. Casati, and D. L. Shepelyansky, Breakdown of universality in renormalization dynamics for critical invariant torus, Europhys. Lett., 15 (1991), pp. 381-386.

[ACS92] R. Artuso, G. Casati, and D. L. Shepelyansky, Break-up of the spiral mean torus in a volume-preserving map, Chaos Solitons Fractals, 2 (1992), pp. 181-190.

[AK03] A. Avila AND R. KRIKORIAn, Reducibility or non-uniform hyperbolicity for quasiperiodic Schrödinger operators, Ann. of Math. (2), 164 (2006), pp. 911-940.

[AS83] J. Avron AND B. Simon, Almost periodic Schrödinger operators. II. The integrated density of states, Duke Math. J., 50 (1983), pp. 369-391.

[AS82] J. Avron And B. Simon, Almost periodic Schrödinger operators. I. Limit periodic potentials, Comm. Math. Phys., $82(1981 / 82)$, pp. 101-120.

[Bjerklov05] K. BJERKLÖv, Positive Lyapunov exponent and minimality for a class of one-dimensional quasiperiodic Schrödinger equations, Ergodic Theory Dynam. Systems, 25 (2005), pp. 1015-1045.

[Bjerklov06] K. BJERKLÖv, Dynamics of the quasi-periodic Schrödinger cocycle at the lowest energy in the spectrum, preprint, 2006.

[BDV04] C. Bonatti, L. Diaz, and M. Viana, Dynamics beyond Uniform Hyperbolicity: A Global Geometric and Probabilistic Perspective, Springer-Verlag, New York, 2004.

$\left[\mathrm{BHJ}^{+} 03\right]$ H. Broer, H. Hanssmann, À. Jorba, J. Villanueva, and F. Wagener, Normal-internal resonances in quasi-periodically forced oscillators: A conservative approach, Nonlinearity, 16 (2003), pp. 1751-1791.

[BhtB90] H. W. Broer, G. B. Huitema, F. Takens, and B. L. J. Branksma, Unfoldings and bifurcations of quasi-periodic tori, Mem. Amer. Math. Soc., 83 (421) (1990).

[BJ02] J. Bourgain And S. Jitomirskaya, Continuity of the Lyapunov exponent for quasiperiodic operators with analytic potential. Dedicated to David Ruelle and Yasha Sinai on the occasion of their 65th birthdays, J. Statist. Phys., 108 (2002), pp. 1203-1218.

[BLW91] D. Beigie, A. Leonard, And S. Wiggins, Chaotic transport in the homoclinic and heteroclinic tangle regions of quasiperiodically forced two-dimensional dynamical systems, Nonlinearity, 4 (1991), pp. 775-819.

[BOV97] H. W. Broer, H. M. Osinga, And G. Vegter, Algorithms for computing normally hyperbolic invariant manifolds, Z. Angew. Math. Phys., 48 (1997), pp. 480-524.

[BS82] J. BÉllissard And B. Simon, Cantor spectrum for the almost Mathieu equation, J. Funct. Anal., 48 (1982), pp. 408-419.

[BS98] H. Broer And C. Simó, Hill's equation with quasi-periodic forcing: Resonance tongues, instability pockets and global phenomena, Bol. Soc. Brasil. Mat. (N.S.), 29 (1998), pp. 253-293.

[BSV05] H. Broer, C. Simó, And R. Vitolo, Chaos and Quasi-Periodicity in Diffeomorphisms of the Solid Torus, MP_ARC \# 05-107, 2005.

[CEY90] M. D. Choi, G. A. Elliott, And N. Yui, Gauss polynomials and the rotation algebra, Invent. Math., 99 (1990), pp. 225-246.

[CFdlL03a] X. Cabré, E. Fontich, and R. DE la Llave, The parameterization method for invariant manifolds. I. Manifolds associated to non-resonant subspaces, Indiana Univ. Math. J., 52 (2003), pp. 283-328.

[CFdlL03b] X. Cabré, E. Fontich, and R. DE la Llave, The parameterization method for invariant manifolds. II. Regularity with respect to parameters, Indiana Univ. Math. J., 52 (2003), pp. 329-360. 
[CFdlL05] X. Cabré, E. Fontich, And R. DE la Llave, The parameterization method for invariant manifolds. III. Overview and applications, J. Differential Equations, 218 (2005), pp. 444515.

[Chi79] B. V. ChIRIKov, A universal instability of many-dimensional oscillator systems, Phys. Rep., 52 (1979), pp. 264-379.

[CJ00] E. CASTELLÀ AND À. JoRBA, On the vertical families of two-dimensional tori near the triangular points of the bicircular problem, Celestial Mech. Dynam. Astronom., 76 (2000), pp. 35-54.

[CL99] C. Chicone And Y. LATushkin, Evolution Semigroups in Dynamical Systems and Differential Equations, AMS, Providence, RI, 1999.

[CL00] C. Chicone AND W. LiU, On the continuation of an invariant torus in a family with rapid oscillations, SIAM J. Math. Anal., 31 (2000), pp. 386-415.

[CLR] Y. Cao, S. Luzzatto, And I. Rios, The Boundary of Hyperbolicity for Henon-Like Families, http://arxiv.org/PS_cache/math/pdf/0502/0502235.pdf.

[CLR06] Y. CAO, S. Luzzatto, AND I. Rios, Some non-hyperbolic systems with strictly non-zero Lyapunov exponents for all invariant measures: Horseshoes with internal tangencies, Discrete Contin. Dyn. Syst., 15 (2006), pp. 61-71.

[DL95] L. Dieci AND J. LoREnz, Computation of invariant tori by the method of characteristics, SIAM J. Numer. Anal., 32 (1995), pp. 1436-1474.

[dlL97] R. DE LA LLAVE, Invariant manifolds associated to nonresonant spectral subspaces, J. Statist. Phys., 87 (1997), pp. 211-249.

[dllGJV05] R. De la Llave, A. GonzÁlez, À. Jorba, And J. Villanueva, KAM theory without actionangle variables, Nonlinearity, 18 (2005), pp. 855-895.

[dlLT94] R. DE LA Llave AND S. Tompaidis, Computation of domains of analyticity for some perturbative expansions of mechanics, Phys. D, 71 (1994), pp. 55-81.

[DLR91] L. Dieci, J. Lorenz, And R. D. Russell, Numerical calculation of invariant tori, SIAM J. Sci. Statist. Comput., 12 (1991), pp. 607-647.

[DS83a] P. DeIFT AND B. Simon, Almost periodic Schrödinger operators. III. The absolutely continuous spectrum in one dimension, Comm. Math. Phys., 90 (1983), pp. 389-411.

[DS83b] F. Delyon AND B. Soulllard, The rotation number for finite difference operators and its properties, Comm. Math. Phys., 89 (1983), pp. 415-426.

[Eli88] L. H. Eliasson, Perturbations of stable invariant tori for Hamiltonian systems, Ann. Scuola Norm. Sup. Pisa Cl. Sci. (4), 15 (1988), pp. 115-147 (1989).

[Eli01] L. H. Eliasson, Almost reducibility of linear quasi-periodic systems, in Smooth Ergodic Theory and Its Applications (Seattle, WA, 1999), Proc. Sympos. Pure Math. 69, AMS, Providence, RI, 2001, pp. 679-705.

[ERS00] K. D. Edoh, R. D. Russell, And W. Sun, Computation of invariant tori by orthogonal collocation, Appl. Numer. Math., 32 (2000), pp. 273-289.

[ESA] ESA, Soho - ESA Space Mission, http://esapub.esrin.esa.it/bulletin/bullet88/vande88.htm.

[Fdil92] C. FAlCOlini AND R. DE la Llave, Numerical calculation of domains of analyticity for perturbation theories in the presence of small divisors, J. Statist. Phys., 67 (1992), pp. 645-666.

[Fen72] N. Fenichel, Persistence and smoothness of invariant manifolds for flows, Indiana Univ. Math. J., 21 (1971/1972), pp. 193-226.

[FJN03a] R. FABBri, R. Johnson, AND C. NúÑEz, Rotation number for non-autonomous linear Hamiltonian systems. I. Basic properties, Z. Angew. Math. Phys., 54 (2003), pp. 484-502.

[FJN03b] R. FABbri, R. Johnson, And C. NúÑEZ, Rotation number for non-autonomous linear Hamiltonian systems. II. The Floquet coefficients, Z. Angew. Math. Phys., 54 (2003), pp. 652-676.

[GC91] A. Griewank And G. F. Corliss, Eds., Automatic Differentiation of Algorithms. Theory, Implementation, and Application, SIAM, Philadelphia, 1991.

[GFPS00] P. Glendinning, U. Feudel, A. S. Pikovsky, and J. Stark, The structure of mode-locked regions in quasi-periodically forced circle maps, Phys. D, 140 (2000), pp. 227-243.

[GJ04] F. GABERN AND À. JoRBA, Generalizing the restricted three-body problem. The bianular and tricircular coherent problems, Astronomy and Astrophysics, 420 (2004), pp. 751-762. 
[GJMS91a] G. Gómez, A. Jorba, J. Masdemont, And C. Simó, A dynamical system approach for the analysis of Soho mission, in Proceedings of the 3rd International Symposium on Spacecraft Flight Dynamics, ESTEC, ESA Publications Division, Noordwijt, Holland, 1991, pp. 449454.

[GJMS91b] G. Gómez, A. Jorba, J. Masdemont, And C. Simó, A quasi-periodic solution as a substitute of $\mathrm{L}_{4}$ in the Earth-Moon system, in Proceedings of the 3rd International Symposium on Spacecraft Flight Dynamics, ESTEC, ESA Publications Division, Noordwijt, Holland, 1991, pp. $35-41$.

[Gre79] J. M. Greene, A method for determining a stochastic transition, J. Math. Phys., 20 (1979), pp. $1183-1201$.

[Har98] À. HARo, The Primitive Function of an Exact Symplectomorphism, Ph.D. thesis, Universitat de Barcelona, Barcelona, Spain, 1998, http://www.maia.ub.es/dsg/1998/index.html.

[Har99] A. HARO, Center and center-(un)stable manifolds of elliptic-hyperbolic fixed points of 4Dsymplectic maps. An example: The Froeschlé map, in Hamiltonian Systems with Three or More Degrees of Freedom (S'Agaró, 1995), Kluwer Academic Publishers, Dordrecht, The Netherlands, 1999, pp. 403-407.

[Har02] À. HARO, An algorithm to generate canonical transformations: Application to normal forms, Phys. D, 167 (2002), pp. 197-217.

[Har05] À. HARo, The Breakdown of Invariant Tori in Quasi-Periodic Area Preserving Maps. An Example: The Rotating Standard Map, work in progress, 2005.

[HdlL03a] A. Haro AND R. DE LA Llave, Spectral Theory of Transfer Operators (I): General Results, preprint, 2003.

[HdlL03b] A. HARO AND R. DE LA Llave, Spectral Theory of Transfer Operators (II): Vector Bundle Maps over Rotations, preprint, 2003.

[HdlL05a] A. HARO AND R. DE LA Llave, A parameterization method for the computation of whiskers in quasi periodic maps: Numerical algorithms, Discrete Contin. Dyn. Syst. Ser. B, 6 (2006), pp. $1261-1300$.

[HdlL05b] A. HARO AND R. DE LA Llave, Invariant manifolds in quasi-periodic systems: Theory, algorithms and computations, in SIAM Conference on Applications of Dynamical Systems, Snowbird, UT, 2005.

[HdlL05c] A. HARO AND R. DE LA LlAVE, Manifolds on the verge of a hyperbolicity breakdown, in SIAM Conference on Applications of Dynamical Systems, Snowbird, UT, 2005.

[HdlL06a] A. HARO AND R. DE LA Llave, Manifolds at the verge of a hyperbolicity breakdown, Chaos, 16 (2006), 012130 .

[HdlL06b] A. HARO AND R. DE LA Llave, A parameterization method for the computation of invariant tori and their whiskers in quasi periodic maps: Rigorous results, J. Differential Equations, 228 (2006), pp. 530-579.

[Her83] M.-R. HeRman, Une méthode pour minorer les exposants de Lyapounov et quelques exemples montrant le caractère local d'un théorème d'Arnol'd et de Moser sur le tore de dimension 2, Comment. Math. Helv., 58 (1983), pp. 453-502.

[Hof76] D. R. Hofstadter, Energy levels and wave functions of Bloch electrons in rational and irrational magnetic fields, Phys. Rev. B (3), 14 (1976), pp. 2239-2245.

[HP05] À. HARO AND J. PUIG, Strange Nonchaotic Attractors in Harper Maps, http://www.arXiv.org/abs/nlin.CD/0510073, 2005.

[HPS77] M. W. Hirsch, C. C. Pugh, And M. Shub, Invariant Manifolds, Lecture Notes in Math. 583, Springer-Verlag, Berlin, 1977.

[HS05] À. HARo AND C. Simó, To Be or Not to Be an SNA: That Is the Question, preprint, 2005-17, University of Barcelona, Barcelona, Spain, 2005.

[JdlLZ99] À. Jorba, R. De la Llave, AND M. Zou, Lindstedt series for lower-dimensional tori, in Hamiltonian Systems with Three or More Degrees of Freedom (S'Agaró, 1995), Kluwer Academic Publishers, Dordrecht, The Netherlands, 1999, pp. 151-167.

[Jit99] S. YA. Jitomirskaya, Metal-insulator transition for the almost Mathieu operator, Ann. of Math. (2), 150 (1999), pp. 1159-1175. 
[JO05] A. Yu. JAlnine And A. H. Osbaldestin, Smooth and nonsmooth dependence of Lyapunov vectors upon the angle variable on a torus in the context of torus-doubling transitions in the quasiperiodically forced Hénon map, Phys. Rev. E (3), 71 (2005), 016206.

[Jor99] À. JoRBA, A methodology for the numerical computation of normal forms, centre manifolds and first integrals of Hamiltonian systems, Experiment. Math., 8 (1999), pp. 155-195.

[Jor01] A. JoRBA, Numerical computation of the normal behaviour of invariant curves of n-dimensional maps, Nonlinearity, 14 (2001), pp. 943-976.

[JS81] R. A. Johnson And G. R. SEll, Smoothness of spectral subbundles and reducibility of quasiperiodic linear differential systems, J. Differential Equations, 41 (1981), pp. 262-288.

[JS92] À. JoRBA AND C. Simó, On the reducibility of linear differential equations with quasiperiodic coefficients, J. Differential Equations, 98 (1992), pp. 111-124.

[JS96] À. Jorba AND C. Simó, On quasi-periodic perturbations of elliptic equilibrium points, SIAM J. Math. Anal., 27 (1996), pp. 1704-1737.

[JT05] A. Jorba AND J. C. TATJER, A Mechanism for the Fractalization of Invariant Curves in QuasiPeriodically Forced 1-D Maps, preprint 2005-12, University of Barcelona, Barcelona, Spain, 2005.

[JV97] À. JoRBa AND J. Villanueva, On the normal behaviour of partially elliptic lower-dimensional tori of Hamiltonian systems, Nonlinearity, 10 (1997), pp. 783-822.

[Kan84] K. KaneKo, Fractalization of torus, Progr. Theoret. Phys., 71 (1984), pp. 1112-1115.

[Kan94] I. KAN, Open sets of diffeomorphisms having two attractors, each with an everywhere dense basin, Bull. Amer. Math. Soc. (N.S.), 31 (1994), pp. 68-74.

[Kat76] T. Kato, Perturbation Theory for Linear Operators, 2nd ed., Grundlehren Math. Wiss. 132, Springer-Verlag, Berlin, 1976.

[Kel96] G. KELLER, A note on strange nonchaotic attractors, Fund. Math., 151 (1996), pp. $139-148$.

[Knu97] D. E. Knuth, The Art of Computer Programming. Vol. 2: Seminumerical Algorithms, 3rd rev. ed., Addison-Wesley, Reading, MA, London, Don Mills, ON, 1997.

[KO98] B. KRAuskopf AND H. OsingA, Growing $1 D$ and quasi-2D unstable manifolds of maps, J. Comput. Phys., 146 (1998), pp. 404-419.

[Koc99] H. KocH, A renormalization group for Hamiltonians, with applications to KAM tori, Ergodic Theory Dynam. Systems, 19 (1999), pp. 1-47.

[Kri99a] R. KRIKORIAN, $C^{0}$-densité globale des systèmes produits-croisés sur le cercle réductibles, Ergodic Theory Dynam. Systems, 19 (1999), pp. 61-100.

[Kri99b] R. KRIKORIAN, Réductibilité des systèmes produits-croisés à valeurs dans des groupes compacts, Astérisque, (259) (1999).

[Kri99c] R. KRIKORIAN, Réductibilité presque partout des flots fibrés quasi-périodiques à valeurs dans des groupes compacts, Ann. Sci. École Norm. Sup. (4), 32 (1999), pp. 187-240.

[Kri01] R. Krikorian, Global density of reducible quasi-periodic cocycles on $\mathbf{T}^{1} \times \mathrm{SU}(2)$, Ann. of Math. (2), 154 (2001), pp. 269-326.

[KSW96] H. Koch, A. Schenkel, AND P. WitTwer, Computer-assisted proofs in analysis and programming in logic: A case study, SIAM Rev., 38 (1996), pp. 565-604.

[Las94] Y. LAST, Zero measure spectrum for the almost Mathieu operator, Comm. Math. Phys., 164 (1994), pp. 421-432.

[LM00] H. E. Lomelí AND J. D. MeIss, Heteroclinic primary intersections and codimension one Melnikov method for volume-preserving maps, in Chaotic Kinetics and Transport (New York, 1998), Chaos, 10 (1) (2000), pp. 109-121.

[LM03] H. E. Lomelí AND J. D. Meiss, Heteroclinic intersections between invariant circles of volumepreserving maps, Nonlinearity, 16 (2003), pp. 1573-1595.

[Mat68] J. N. Mather, Characterization of Anosov diffeomorphisms, Nederl. Akad. Wetensch. Proc. Ser. A 71 = Indag. Math., 30 (1968), pp. 479-483.

[Mei92] J. D. MeIss, Symplectic maps, variational principles, and transport, Rev. Modern Phys., 64 (1992), pp. 795-848.

[MKM97] H. Mingyou, T. Küpper, And N. Masbaum, Computation of invariant tori by the Fourier methods, SIAM J. Sci. Comput., 18 (1997), pp. 918-942. 
[Mn78] R. MAÑÉ, Persistent manifolds are normally hyperbolic, Trans. Amer. Math. Soc., 246 (1978), pp. 261-283.

[MO00] B. D. Mestel And A. H. Osbaldestin, Periodic orbits of renormalisation for the correlations of strange nonchaotic attractors, Math. Phys. Electron. J., 6 (2000), paper 5.

[MO04] B. D. Mestel And A. H. Osbaldestin, Renormalization of correlations in a quasiperiodically forced two-level system: Quadratic irrationals, J. Phys. A, 37 (2004), pp. 8969-8989.

[MS91] K. R. Meyer And D. S. Schmidt, Eds., Computer Aided Proofs in Analysis, IMA Vol. Math. Appl. 28, Springer-Verlag, New York, 1991.

[NAS] NASA, Genesis - NASA Space Mission, http://spacelink.msfc.nasa.gov/NASA.Projects.

[NK96] T. NishikAWA AND K. KANEKO, Fractalization of a torus as a strange nonchaotic attractor, Phys. Rev. E (3), 54 (1996), pp. 6114-6124.

[NNO98] S. Novo, C. NúÑEz, AND R. OBAYA, Ergodic properties and rotation number for linear Hamiltonian systems, J. Differential Equations, 148 (1998), pp. 148-185.

[OF00] H. M. Osinga AND U. Feudel, Boundary crisis in quasiperiodically forced systems, Phys. D, 141 (2000), pp. 54-64.

[OM03] A. H. Osbaldestin And B. D. Mestel, Renormalization in quasiperiodically forced systems, Fluct. Noise Lett., 3 (2003), pp. L251-L258.

[OS87] A. Olvera AND C. Simó, An obstruction method for the destruction of invariant curves, Phys. D, 26 (1987), pp. 181-192.

[Ose68] V. I. Oseledec, A multiplicative ergodic theorem. Characteristic Lyapunov, exponents of dynamical systems, Trudy Moskov. Mat. Obšč., 19 (1968), pp. 179-210.

[OWGF01] H. Osinga, J. Wiersig, P. Glendinning, And U. Feudel, Multistability and nonsmooth bifurcations in the quasiperiodically forced circle map, Internat. J. Bifur. Chaos Appl. Sci. Engrg., 11 (2001), pp. 3085-3105.

[Pes77] JA. B. Pesin, Characteristic Lyapunov exponents, and smooth ergodic theory, Russian Math. Surveys, 32 (1977), pp. 55-114.

[PNR01] A. Prasad, S. S. Negi, And R. Ramaswamy, Strange nonchaotic attractors, Internat. J. Bifur. Chaos Appl. Sci. Engrg., 11 (2001), pp. 291-309.

[Pös89] J. Pöschel, On elliptic lower-dimensional tori in Hamiltonian systems, Math. Z., 202 (1989), pp. 559-608.

[Pui04] J. Puig, Cantor spectrum for the almost Mathieu operator, Comm. Math. Phys., 244 (2004), pp. 297-309.

[Pui05] J. Puig, A nonperturbative Eliasson's reducibility theorem, Nonlinearity, 19 (2006), pp. 335-376.

[RJB83] R. R. Ricklefs, W. JefFerys, AND R. Broucke, A general precompiler for algebraic manipulation, Celest. Mech., 29 (1983), pp. 179-190.

[Rue85] D. Ruelle, Rotation numbers for diffeomorphisms and flows, Ann. Inst. H. Poincaré Phys. Théor., 42 (1985), pp. 109-115.

[Ryc92] M. RYCHLIK, Renormalization of cocycles and linear ODE with almost-periodic coefficients, Invent. Math., 110 (1992), pp. 173-206.

[Sac78] R. J. SACKER, Existence of dichotomies and invariant splittings for linear differential systems. IV, J. Differential Equations, 27 (1978), pp. 106-137.

[SFKA96] O. Sosnovtseva, U. Feudel, J. Kurths, And A. Pikovsky, Multiband strange attractors in quasiperiodically forced systems, Phys. Lett. A, 218 (1996), pp. 255-272.

[Sim79] C. Simó, On the Hénon-Pomeau attractor, J. Statist. Phys., 21 (1979), pp. 465-494.

[Sim98] C. Simó, Effective computations in celestial mechanics and astrodynamics, in Modern Methods of Analytical Mechanics and Their Applications (Udine, 1997), V. V. Rumynantsev and A. V. Karapatian, eds., Springer-Verlag, Vienna, 1998, pp. 55-102.

[Sim99] C. Simó, Dynamical systems methods for space missions on a vicinity of collinear libration points, in Hamiltonian Systems with Three or More Degrees of Freedom (S'Agaró, 1995), Kluwer Academic Publishers, Dordrecht, The Netherlands, 1999, pp. 223-241.

[Sta99] J. STARK, Regularity of invariant graphs for forced systems, Ergodic Theory Dynam. Systems, 19 (1999), pp. 155-199.

[Thi97] Ph. Thieullen, Ergodic reduction of random products of two-by-two matrices, J. Anal. Math., 73 (1997), pp. 19-64. 
[Tom96] S. TOMPAIDIS, Numerical study of invariant sets of a quasiperiodic perturbation of a symplectic map, Experiment. Math., 5 (1996), pp. 211-230.

[Tru00] M. R. Trummer, Spectral methods in computing invariant tori, in Auckland Numerical Ordinary Differential Equations (Auckland, 1998), Appl. Numer. Math., 34 (2-3) (2000), pp. 275-292.

[WR87] R. L. WARNOCK AND R. D. Ruth, Invariant tori through direct solution of the Hamilton-Jacobi equation, Phys. D, 26 (1987), pp. 1-36.

[WY01] Q. WAng And L.-S. Young, Strange attractors with one direction of instability, Comm. Math. Phys., 218 (2001), pp. 1-97.

[WY02] Q. WANG AND L.-S. Young, From invariant curves to strange attractors, Comm. Math. Phys., 225 (2002), pp. 275-304. 